\title{
Pd-Catalyzed Enantioselective Hydroalkynylation of Cyclopropenes
}

\author{
Longyang Dian, Ilan Marek*
}

Schulich Faculty of Chemistry, Technion-Israel Institute of Technology, Technion City, Haifa, 32000, Israel

* Corresponding Author

E-mail: chilanm@technion.ac.il

\section{Contents}

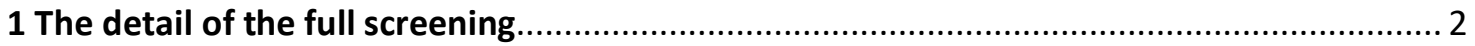

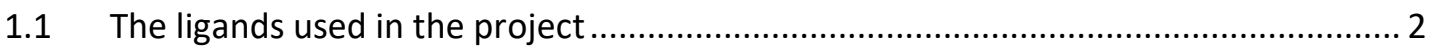

1.2 Table S1. Screening of different chiral ligands............................................................... 3

1.3 Table S2. Screening of different solvents..................................................................... 4

1.4 Table S3. Screening of different Palladium salts.............................................................. 5

1.5 Table S4. Screening of different solvents with $\mathrm{Pd}(\mathrm{acac})_{2}$ as catalyst............................... 6

2. General procedure for the asymmetric Pd-catalyzed hydroalkynylation of cyclopropenes 7

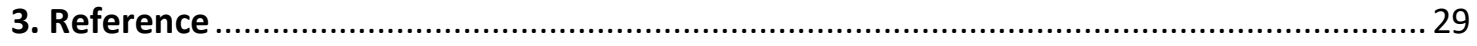

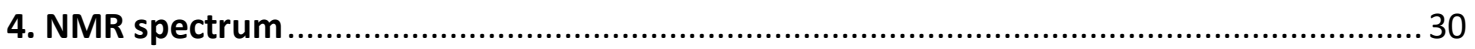

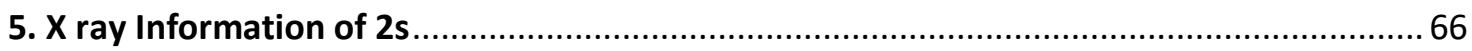




\section{The detail of the full screening}

\subsection{The ligands used in the project}

\section{Chiral ligands}<smiles>c1ccc(-c2ccccc2-c2c(-c3ccccc3)ccc3ccccc23)cc1</smiles>

(R)-BINAP<smiles>c1ccc(-c2ccc3c(c2-c2c(P(c4ccccc4)c4ccccc4)ccc4c2CCCC4)CCCC3)cc1</smiles>

(R)- $\mathrm{H}_{8}$-BINAP<smiles>O=[P+]([O-])c1ccc2ccccc2c1-c1c([Pb](=O)[O-])ccc2ccccc12</smiles>

(R)-p-Tol-BINAP<smiles>Cc1cc(C)cc(P(c2cc(C)cc(C)c2)c2ccc3ccccc3c2-c2c(P(c3cc(C)cc(C)c3)c3cccc4ccccc34)ccc3ccccc23)c1</smiles>

(R)-DM-BINAP<smiles>CC(C1CCC1)P(C1CCCC1)C1CCCC1P(c1ccccc1)c1ccccc1</smiles><smiles>c1ccc(C2[C@H](c3ccccc3)CCP2CCCP2C(c3ccccc3)CCC2c2ccccc2)cc1</smiles>

$(S, S)-P h B P E$<smiles>COc1cccc(P(c2ccco2)c2ccco2)c1-c1ccccc1P(c1ccco1)c1ccco1</smiles>

$(R, S)$-JOSIPHOS EtOH<smiles>c1ccc(-c2ccc3c(c2-c2c(-c4ccccc4)ccc4c2OCO4)OCO3)cc1</smiles>

(R)-SEGPHOS<smiles>IOc1cccc2c1[C@]1(CC2)CCI2OOc3cccc1c32</smiles>

$15-5157$ 15-0112<smiles>CC(c1ccccc1)N(C(C)c1ccccc1)P(Oc1ccc2ccccc2c1-c1ccccc1)c1c(O)ccc2ccccc12</smiles>

$(S, R, R)$-phosphoramidite ligand

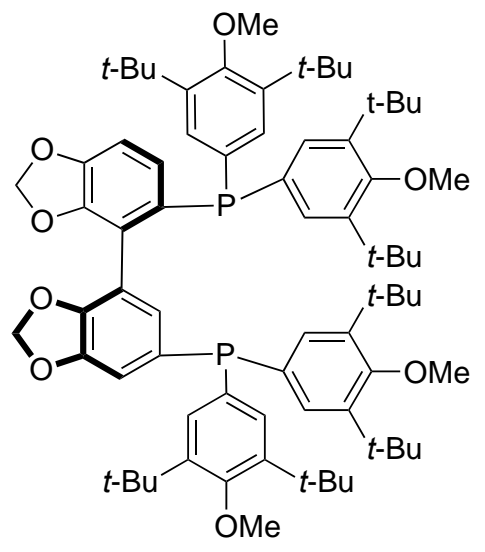

(R)-DTBM-SEGPHOS<smiles>COc1ccc2c(c1)-c1cc(cc(C(C)(C)C)c1OC)Pc1cc(C(C)(C)C)cc(c1OC)Pc1cc(C(C)(C)C)cc(c1OC)C(C(C)(C)C)c1cc(cc(C(C)(C)C)c1OC)P2</smiles>

15-0042 


\subsection{Table S1. Screening of different chiral ligands.}

\begin{tabular}{|c|c|c|c|c|}
\hline entry & cat. & ligand & solvent & $\mathrm{Er}$ \\
\hline 1 & $\mathrm{Pd}(\mathrm{OAc})_{2}$ & $(R)$-BINAP & DCE & $70: 30$ \\
\hline 2 & $\mathrm{Pd}(\mathrm{OAc})_{2}$ & (S)-DTBM-SEGPHOS & DCE & $36: 64$ \\
\hline 3 & $\mathrm{Pd}(\mathrm{OAc})_{2}$ & $(R)-\mathrm{H}_{8}-\mathrm{BINAP}$ & DCE & $85: 15$ \\
\hline 4 & $\mathrm{Pd}(\mathrm{OAc})_{2}$ & $(R)$-Tol-BINAP & DCE & $71: 29$ \\
\hline 5 & $\mathrm{Pd}(\mathrm{OAc})_{2}$ & $(R)$-SEGPHOS & DCE & ND \\
\hline 6 & $\mathrm{Pd}(\mathrm{OAc})_{2}$ & $(R, S)$-Josiphos & DCE & ND \\
\hline 7 & $\mathrm{Pd}(\mathrm{OAc})_{2}$ & $(S, S)-\mathrm{PhBPE}$ & DCE & ND \\
\hline 8 & $\mathrm{Pd}(\mathrm{OAc})_{2}$ & $(R, S, S)$ - Phosphoramidites & DCE & $57: 43$ \\
\hline 9 & $\mathrm{Pd}(\mathrm{OAc})_{2}$ & Trost ligand & DCE & ND \\
\hline 10 & $\mathrm{Pd}(\mathrm{OAc})_{2}$ & (R)-DM-BINAP & DCE & $86: 14$ \\
\hline 11 & $\mathrm{Pd}(\mathrm{OAc})_{2}$ & $15-5157$ & DCE & ND \\
\hline 12 & $\mathrm{Pd}(\mathrm{OAc})_{2}$ & $15-0112$ & DCE & $84: 16$ \\
\hline 13 & $\mathrm{Pd}(\mathrm{OAc})_{2}$ & $15-0042$ & DCE & $70: 30$ \\
\hline 14 & $\mathrm{Pd}(\mathrm{OAc})_{2}$ & $15-0654$ & DCE & $55: 45$ \\
\hline
\end{tabular}

The reactions were run on a $0.05 \mathrm{mmol}$ scale, Pd salt $(5 \mathrm{~mol} \%), \mathrm{L}^{*}(7.5 \mathrm{~mol} \%)$ in DCE (0.1 $\mathrm{M})$ and the reaction mixture was stirred at room temperature for $16 \mathrm{~h}$. The enantiomeric excess has been determined by chiral HPLC. ND: No detection of the desired product $\mathbf{2 a}$. 


\subsection{Table S2. Screening of different solvents.}

\begin{tabular}{|c|c|c|c|c|}
\hline entry & cat. & chiral ligand & solvent & $\mathrm{Er}$ \\
\hline 1 & $\mathrm{Pd}(\mathrm{OAc})_{2}$ & $(R)$-DM-BINAP & $\mathrm{DCE}$ & $86: 14$ \\
\hline 2 & $\mathrm{Pd}(\mathrm{OAc})_{2}$ & $(R)$-DM-BINAP & DCM & $94: 06$ \\
\hline 3 & $\mathrm{Pd}(\mathrm{OAc})_{2}$ & $(R)$-DM-BINAP & $\mathrm{MeCN}$ & ND \\
\hline 4 & $\mathrm{Pd}(\mathrm{OAc})_{2}$ & $(R)$-DM-BINAP & toluene & ND \\
\hline 5 & $\mathrm{Pd}(\mathrm{OAc})_{2}$ & $(R)$-DM-BINAP & $\mathrm{Et}_{2} \mathrm{O}$ & 93:07 \\
\hline 6 & $\mathrm{Pd}(\mathrm{OAc})_{2}$ & $(R)$-DM-BINAP & THF & 94:06 \\
\hline 7 & $\mathrm{Pd}(\mathrm{OAc})_{2}$ & $(R)$-DM-BINAP & DMF & $75: 25$ \\
\hline 8 & $\mathrm{Pd}(\mathrm{OAc})_{2}$ & $(R)$-DM-BINAP & Dioxane & $88: 12$ \\
\hline 9 & $\mathrm{Pd}(\mathrm{OAc})_{2}$ & $(R)$-DM-BINAP & DMSO & $92: 08$ \\
\hline
\end{tabular}

The reactions were run on a $0.05 \mathrm{mmol}$ scale, Pd salt (5 mol\%), (R)-DM-BINAP (7.5 mol\%) in the corresponding solvent $(0.1 \mathrm{M})$ and the reaction mixture was stirred at room temperature for $16 \mathrm{~h}$. The enantiomeric excess has been determined by chiral HPLC. ND: No detection of the desired product $\mathbf{2 a}$. 


\subsection{Table S3. Screening of different Palladium salts.}

\begin{tabular}{|c|c|c|c|c|}
\hline entry & cat. & chiral ligand & solvent & $\mathrm{Er}$ \\
\hline 1 & $\mathrm{Pd}(\mathrm{OAc})_{2}$ & $(R)$-DM-BINAP & DCM & $90: 10$ \\
\hline 2 & $\mathrm{PdCl}_{2}$ & $(R)$-DM-BINAP & DCM & NA \\
\hline 3 & $\mathrm{Pd}(\mathrm{TFA})_{2}$ & $(R)$-DM-BINAP & DCM & NA \\
\hline 4 & $\mathrm{Pd}(\mathrm{OTs})_{2}$ & $(R)$-DM-BINAP & DCM & NA \\
\hline 5 & $\operatorname{Pd}(\text { acac })_{2}$ & $(R)$-DM-BINAP & DCM & 95:05 \\
\hline 6 & $\mathrm{Pd}(\mathrm{dpa})_{2}$ & $(R)$-DM-BINAP & DCM & $93: 07$ \\
\hline 7 & $\mathrm{Pd}(\mathrm{dppf})_{2} \mathrm{Cl}_{2}$ & $(R)$-DM-BINAP & DCM & NA \\
\hline 8 & $(\mathrm{PdAllyCl})_{2}$ & $(R)$-DM-BINAP & DCM & $60: 40$ \\
\hline 9 & $\mathrm{Pd} / \mathrm{CaCO}_{3}$ & $(R)$-DM-BINAP & DCM & NA \\
\hline
\end{tabular}

The reactions were run on a $0.05 \mathrm{mmol}$ scale, $\mathrm{Pd}$ salt $(5 \mathrm{~mol} \%), \mathrm{L}^{*}(7.5 \mathrm{~mol} \%)$ in the corresponding solvent $(0.1 \mathrm{M})$ and the reaction mixture was stirred at room temperature for $16 \mathrm{~h}$. The enantiomeric excess has been determined by chiral HPLC. NA: no analysis of the er because of no detection or trace amount of $\mathbf{2 a}$ was detected in the indicated condition. 


\subsection{Table S4. Screening of different solvents with Pd(acac)2 as} catalyst.

\begin{tabular}{|c|c|c|c|c|}
\hline entry & cat. & chiral ligand & solvent & $\mathrm{Er}$ \\
\hline 1 & $\mathrm{Pd}(\mathrm{acac})_{2}$ & $(R)$-DM-BINAP & DCE & $86: 14$ \\
\hline 2 & $\mathrm{Pd}(\mathrm{acac})_{2}$ & $(R)$-DM-BINAP & DCM & $95: 05$ \\
\hline 3 & $\mathrm{Pd}(\mathrm{acac})_{2}$ & $(R)$-DM-BINAP & $\mathrm{MeCN}$ & NA \\
\hline 4 & $\mathrm{Pd}(\mathrm{acac})_{2}$ & $(R)$-DM-BINAP & toluene & NA \\
\hline 5 & $\mathrm{Pd}(\mathrm{acac})_{2}$ & $(R)$-DM-BINAP & $\mathrm{Et}_{2} \mathrm{O}$ & 98:02 \\
\hline 6 & $\mathrm{Pd}(\mathrm{acac})_{2}$ & $(R)$-DM-BINAP & THF & $96: 04$ \\
\hline 7 & $\mathrm{Pd}(\mathrm{acac})_{2}$ & $(R)$-DM-BINAP & DMF & NA \\
\hline 8 & $\mathrm{Pd}(\mathrm{acac})_{2}$ & $(R)$-DM-BINAP & DMSO & NA \\
\hline 9 & $\mathrm{Pd}(\mathrm{acac})_{2}$ & (R)-DM-BINAP & acetone & 93:07 \\
\hline
\end{tabular}

The reactions were run on a $0.05 \mathrm{mmol}$ scale, $\mathrm{Pd}$ salt $(5 \mathrm{~mol} \%), \mathrm{L}^{*}(7.5 \mathrm{~mol} \%)$ in the corresponding solvent $(0.1 \mathrm{M})$ and the reaction mixture was stirred at room temperature for $16 \mathrm{~h}$. The enantiomeric excess has been determined by chiral HPLC. NA: no analysis of the er because of no detection or trace amount of $2 \mathbf{a}$ was detected in the indicated condition. 


\section{General procedure for the asymmetric Pd-catalyzed hydroalkynylation of cyclopropenes}

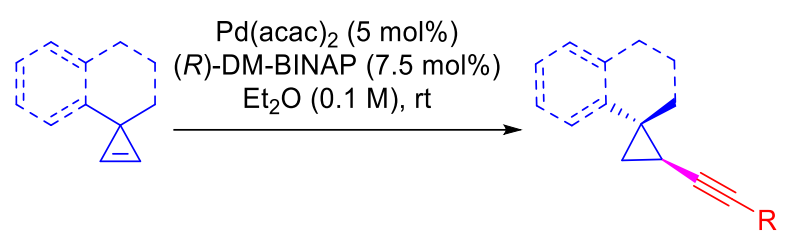

General Procedure: The Pd(acac) 2 salt $(3.0 \mathrm{mg}, 0.010 \mathrm{mmol}, 5 \mathrm{~mol} \%)$ and $(R)$-DMBINAP $(11.0 \mathrm{mg}, 0.015 \mathrm{mmol}, 7.5 \mathrm{~mol} \%)$ was added to a flask charged with a stirring bar, $\mathrm{Et}_{2} \mathrm{O}(2 \mathrm{~mL}, 0.1 \mathrm{M})$ was then injected to the flask under $\mathrm{N}_{2}$ atmosphere. After stirring at room temperature for $10 \mathrm{~min}$, the corresponding alkyne $(0.40 \mathrm{mmol}, 2.0$ equiv.) was added to the flask, followed by the dropwise addition of corresponding cyclopropene $(0.20 \mathrm{mmol})$. The reaction mixture was stirred at room temperature and the process was minotored by TLC, the reaction time is about $16 \mathrm{~h}$ (for aryl acetylene) or $36 \mathrm{~h}$ (for aryl enynes). The reaction mixture was evaporated directly and the oily residue was purified by flash column chromatography on silica gel with petroleum ether (PE)/diether ether (100\%-99\% PE) as eluents to afford the corresponding product.

\section{((1S,2S)-1-Methyl-2-(phenylethynyl)cyclopropyl)benzene (2a)1}

Alkynylated cyclopropane 2a was prepared according to the general procedure. After standard work-up and purification $\mathbf{2 a}$ was obtained as a colorless oil $(37.6 \mathrm{mg}, 75 \%$ yield).

$1 \mathbf{H}$ NMR $\left(400 \mathrm{MHz}, \mathrm{CDCl}_{3}\right) \delta 7.39-7.32(\mathrm{~m}, 2 \mathrm{H}), 7.28-7.19(\mathrm{~m}, 7 \mathrm{H}), 7.17-7.11(\mathrm{~m}$, $1 \mathrm{H}), 1.76(\mathrm{dd}, J=8.9,5.6 \mathrm{~Hz}, 1 \mathrm{H}), 1.55(\mathrm{~s}, 3 \mathrm{H}), 1.42(\mathrm{dd}, J=8.9,4.5 \mathrm{~Hz}, 1 \mathrm{H}), 0.97$ (dd, $J=5.7,4.5 \mathrm{~Hz}, 1 \mathrm{H}$ ). Chiral HPLC analysis (CHIRALCEL IC, 100\% hexane, 1.0 $\mathrm{mL} / \mathrm{min}, 254 \mathrm{~nm}, \mathrm{tR} 1=9.7 \mathrm{~min}, \mathrm{tR} 2=10.7 \mathrm{~min})$ indicated $98: 02 \mathrm{er} .[\alpha] \mathrm{D} 20=+288.79(\mathrm{c}=$ $\left.0.76, \mathrm{CH}_{2} \mathrm{Cl}_{2}\right)$.

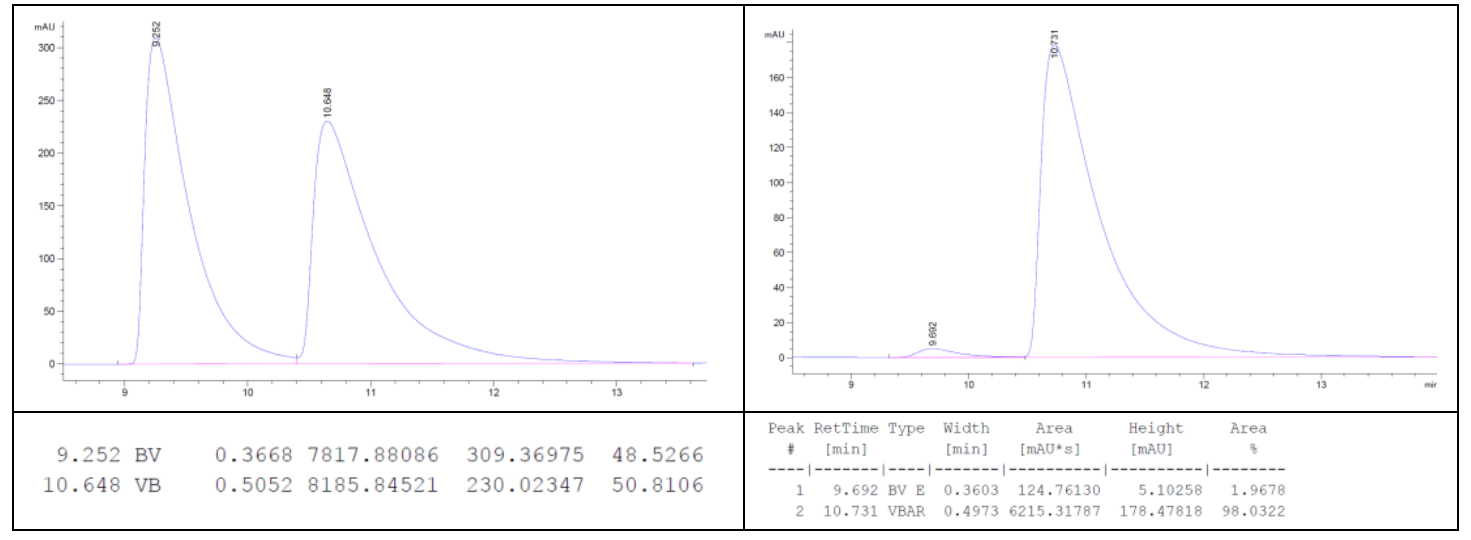

1-Chloro-4-((1S,2S)-1-methyl-2-(phenylethynyl)cyclopropyl)benzene (2b) 
Alkynylated cyclopropane $\mathbf{2 b}$ was prepared according to the general procedure. After standard work-up and purification, $\mathbf{2 b}$ was obtained as a colorless oil $(47.5 \mathrm{mg}, 89 \%$ yield).

1H NMR (400 MHz, CDCl 3$) \delta 7.54-7.45(\mathrm{~m}, 2 \mathrm{H}), 7.39-7.26(\mathrm{~m}, 7 \mathrm{H}), 1.86(\mathrm{dd}, J=$ 9.0, $5.7 \mathrm{~Hz}, 1 \mathrm{H}), 1.67(\mathrm{~s}, 3 \mathrm{H}), 1.51(\mathrm{dd}, J=9.0,4.7 \mathrm{~Hz}, 1 \mathrm{H}), 1.11(\mathrm{dd}, J=5.7,4.6 \mathrm{~Hz}$, 1H). 13C NMR (100 MHz, $\left.\mathrm{CDCl}_{3}\right) \delta 144.1,132.0,131.6,128.7,128.5,128.2,127.6$, 123.8, 89.9, 79.6, 27.4, 23.2, 22.5, 16.1. HRMS (TOF-MS ES+): [M+H]+, calculated for $\mathrm{C}_{18} \mathrm{H}_{16} \mathrm{Cl}$ : 267.0935; found 267.0907.

Chiral HPLC (CHIRALCEL OD, 100\% hexane, $1.0 \mathrm{~mL} / \mathrm{min}, 254 \mathrm{~nm}$, tR $1=10.6 \mathrm{~min}$, tR2 $=12.9 \mathrm{~min})$ indicated 96:04 er. $[\alpha] \mathbf{D 2 0}=+338.20\left(\mathrm{c}=1.10, \mathrm{CH}_{2} \mathrm{Cl}_{2}\right)$.

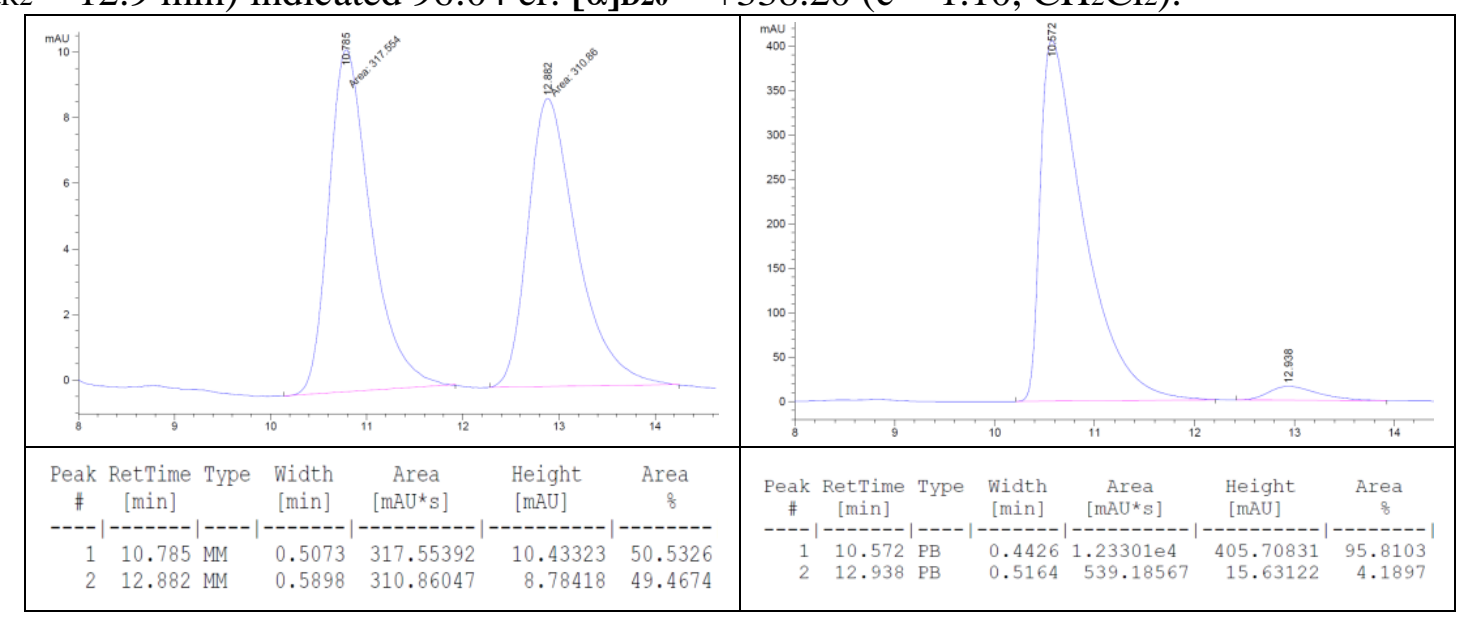

1-Methyl-4-((1S,2S)-1-methyl-2-(phenylethynyl)cyclopropyl)benzene (2c)

Alkynylated cyclopropane 2c was prepared according to the general procedure. After standard work-up and purification, $2 \mathbf{c}$ was obtained as a colorless oil $(30.5 \mathrm{mg}, 62 \%$ yield).

1H NMR (400 MHz, CDCl $) \delta$ 7.50-7.47 (m, 2H), 7.35-7.33 (m, 3H), $7.27(\mathrm{~d}, J=8.0$ $\mathrm{Hz}, 2 \mathrm{H}), 7.18(\mathrm{~d}, J=8.0 \mathrm{~Hz}, 2 \mathrm{H}), 2.40-2.33(\mathrm{~m}, 4 \mathrm{H}), 1.86(\mathrm{dd}, J=8.9,5.6 \mathrm{~Hz}, 1 \mathrm{H}), 1.51$ (dd, $J=4.5,9.0 \mathrm{~Hz}, 1 \mathrm{H}), 1.07$ (dd, $J=4.5,5.5 \mathrm{~Hz}, 1 \mathrm{H})$.

13C NMR (100 MHz, $\left.\mathrm{CDCl}_{3}\right) \delta 142.7,135.9,131.6,129.1,128.2,127.5,127.2,124.0$, 90.6, 79.2, 27.7, 23.2, 22.7, 21.0, 15.9. HRMS (TOF-MS ES+): [M+H] $]_{+}$, calculated for $\mathrm{C}_{19} \mathrm{H}_{19}$ : 247.1481; found 247.1495.

Chiral HPLC (CHIRALCEL IA, 100\% hexane, $1.0 \mathrm{~mL} / \mathrm{min}, 254 \mathrm{~nm}$, tR $1=8.1 \mathrm{~min}$, tR2 $=9.0 \mathrm{~min})$ indicated 97:03 er. $[\alpha]_{\mathbf{D} 20}=+206.72\left(\mathrm{c}=0.40, \mathrm{CH}_{2} \mathrm{Cl}_{2}\right)$. 


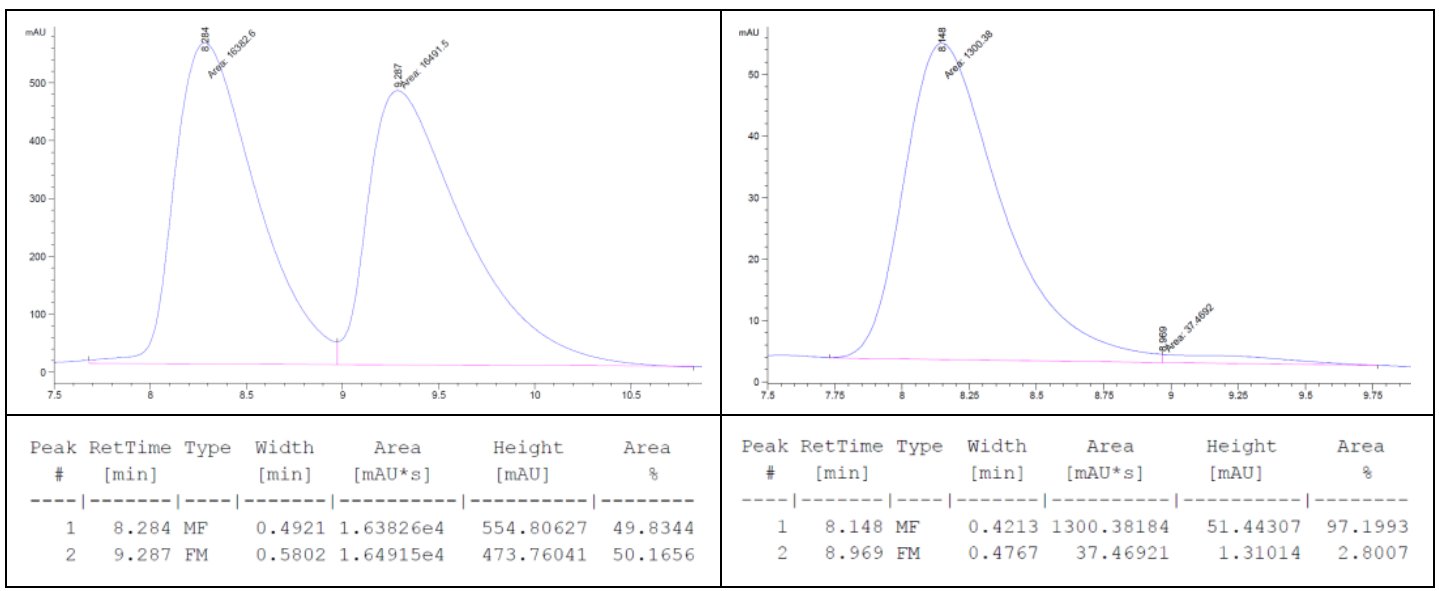

\section{1-Methoxy-4-((1S,2S)-1-methyl-2-(phenylethynyl)cyclopropyl)benzene (2d)}

Alkynylated cyclopropane 2d was prepared according to the general procedure. After standard work-up and purification, 2d was obtained as a colorless oil $(32.0 \mathrm{mg}, 61 \%$ yield).

1H NMR (400 MHz, CDCl 3$) \delta 7.39-7.31(\mathrm{~m}, 2 \mathrm{H}), 7.19(\mathrm{dd}, J=17.8,7.2 \mathrm{~Hz}, 5 \mathrm{H}), 6.77$ $(\mathrm{d}, J=8.6 \mathrm{~Hz}, 2 \mathrm{H}), 3.71(\mathrm{~s}, 3 \mathrm{H}), 1.70(\mathrm{dd}, J=8.9,5.6 \mathrm{~Hz}, 1 \mathrm{H}), 1.52(\mathrm{~s}, 3 \mathrm{H}), 1.36(\mathrm{t}, J=$ $4.4 \mathrm{~Hz}, 1 \mathrm{H}), 0.93(\mathrm{t}, J=5.0 \mathrm{~Hz}, 1 \mathrm{H}) .{ }_{13} \mathrm{C} \mathrm{NMR}\left(100 \mathrm{MHz}, \mathrm{CDCl}_{3}\right) \delta 158.0,137.9,131.6$, $128.5,128.2$, 127.5, 124.0, 113.7, 90.6, 79.2, 55.3, 27.5, 23.2, 22.9, 15.7. HRMS (TOFMS ES+): [M+H] $]_{+}$, calculated for $\mathrm{C}_{19} \mathrm{H}_{19} \mathrm{O}$ : 263.1430; found 263.1439.

Chiral HPLC (CHIRALCEL IA, 99\% hexane, $1.0 \mathrm{~mL} / \mathrm{min}, 254 \mathrm{~nm}$, tR1 $=9.9 \mathrm{~min}$, tR2 = $10.1 \mathrm{~min})$ indicated 98:02 er. $[\alpha] \mathbf{D 2 0}=+213.67\left(\mathrm{c}=0.23, \mathrm{CH}_{2} \mathrm{Cl}_{2}\right)$.

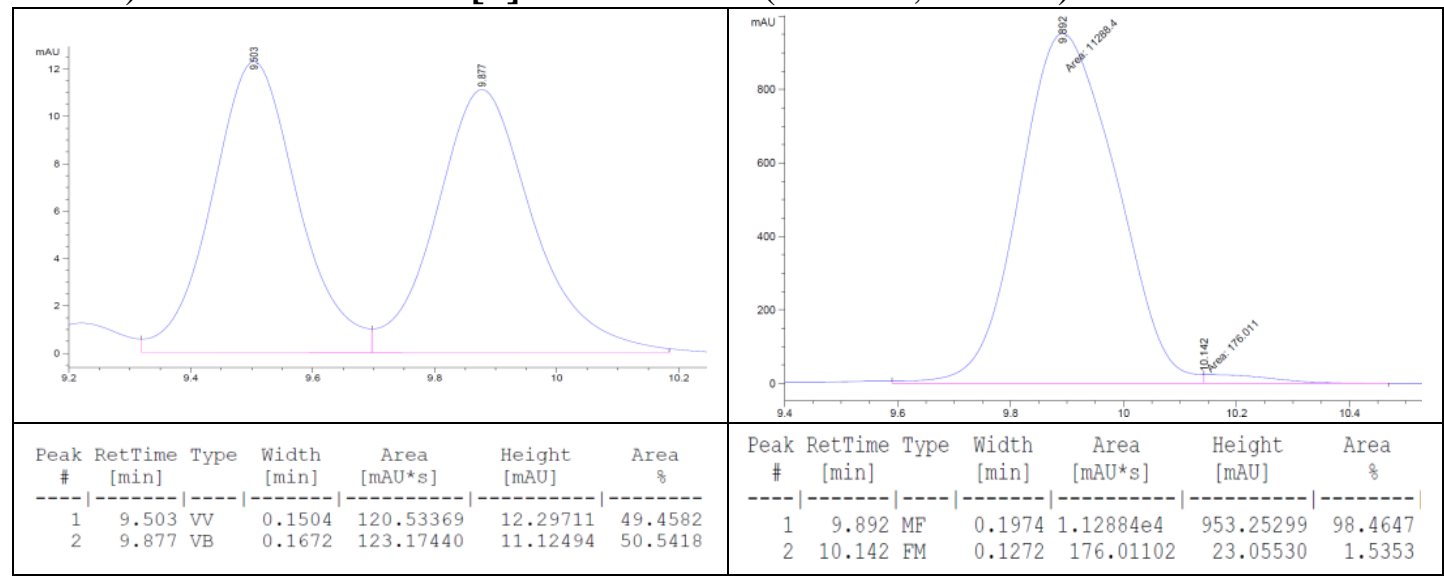

Alkynylated cyclopropane $\mathbf{2} \mathbf{e} / \mathbf{2} \mathbf{e}^{\prime}$ was prepared according to the general procedure 1 . After standard work-up and purification, $\mathbf{2 e}$ and $\mathbf{2} \mathbf{e}^{\prime}$ were obtained as a colorless oil, which could be sepearated by column chromatography (2e, $15.8 \mathrm{mg}$, 32\% yield; 2e', $15.3 \mathrm{mg}, 31 \%$ yield). 


\section{(((1S,2S)-2-Benzyl-2-methylcyclopropyl)ethynyl)benzene (2e)}

1H NMR (400 MHz, CDCl3) $\delta 7.36-7.09(\mathrm{~m}, 10 \mathrm{H}), 2.69-2.50(\mathrm{~m}, 2 \mathrm{H}), 1.45(\mathrm{dd}, J=$ $8.8,5.3 \mathrm{~Hz}, 1 \mathrm{H}), 1.16(\mathrm{~s}, 3 \mathrm{H}), 0.98(\mathrm{dd}, J=8.8,4.4 \mathrm{~Hz}, 1 \mathrm{H}), 0.62(\mathrm{t}, J=4.8 \mathrm{~Hz}, 1 \mathrm{H}) .13 \mathrm{C}$ NMR (100 MHz, $\left.\mathrm{CDCl}_{3}\right) \delta 139.2,131.6,129.3,128.2,128.2,128.2,128.1,127.4,126.3$, 124.1, 91.1, 78.5, 45.0, 24.3, 21.9, 19.4, 13.4. HRMS (TOF-MS ES+): [M+H]+, calculated for $\mathrm{C}_{19} \mathrm{H}_{19}$ : 247.1481; found 247.1432.

Chiral HPLC analysis (CHIRALCEL IA, 100\% hexane, $1.0 \mathrm{~mL} / \mathrm{min}, 254 \mathrm{~nm}, \mathrm{tR} 1=8.2$ min, tR2 $=9.5 \mathrm{~min})$ indicated $96: 04 \mathrm{er}$. $[\alpha] \mathbf{D 2 0}=+127.17\left(\mathrm{c}=0.33, \mathrm{CH}_{2} \mathrm{Cl}_{2}\right)$.

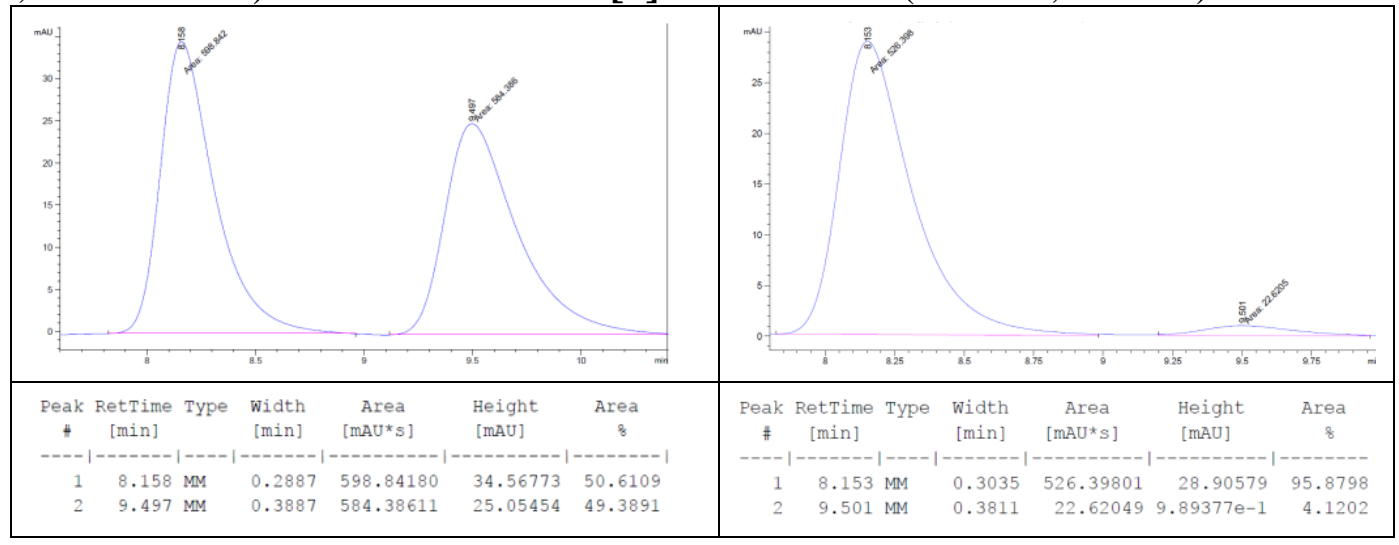

\section{(((1S,2R)-2-Benzyl-2-methylcyclopropyl)ethynyl)benzene (2e')}

$\mathbf{2} \mathbf{e}^{\prime}$ was obtained as a colorless oil $(15.3 \mathrm{mg}, 31 \%$ yield) $\mathbf{1 H}$ NMR (400 $\mathbf{~ M H z}, \mathbf{C D C l} 3) \delta$ $7.36-7.10(\mathrm{~m}, 10 \mathrm{H}), 2.97-2.71(\mathrm{~m}, 2 \mathrm{H}), 1.40(\mathrm{dd}, J=8.3,5.6 \mathrm{~Hz}, 1 \mathrm{H}), 0.93(\mathrm{~s}, 3 \mathrm{H})$, $0.91-0.84$ (m, 2H). 13C NMR (100 MHz, CDCl$) \delta$ 140.2, 131.6, 129.3, 128.2, 128.2, 127.5, 126.0, 124.0, 91.5, 79.3, 41.2, 24.9, 22.9, 22.9, 14.7. HRMS (TOF-MS ES+): [M+H]+, calculated for $\mathrm{C}_{19} \mathrm{H}_{19}$ : 247.1481; found 247.1455.

Chiral HPLC analysis (CHIRALCEL IA, 100\% hexane, $1.0 \mathrm{~mL} / \mathrm{min}, 254 \mathrm{~nm}$, tR1 $=7.7$ $\mathrm{min}, \mathrm{tR} 2=7.0 \mathrm{~min})$ indicated $91: 09 \mathrm{er} .[\alpha] \mathrm{D20}=+144.75\left(\mathrm{c}=0.38, \mathrm{CH}_{2} \mathrm{Cl} 2\right)$.

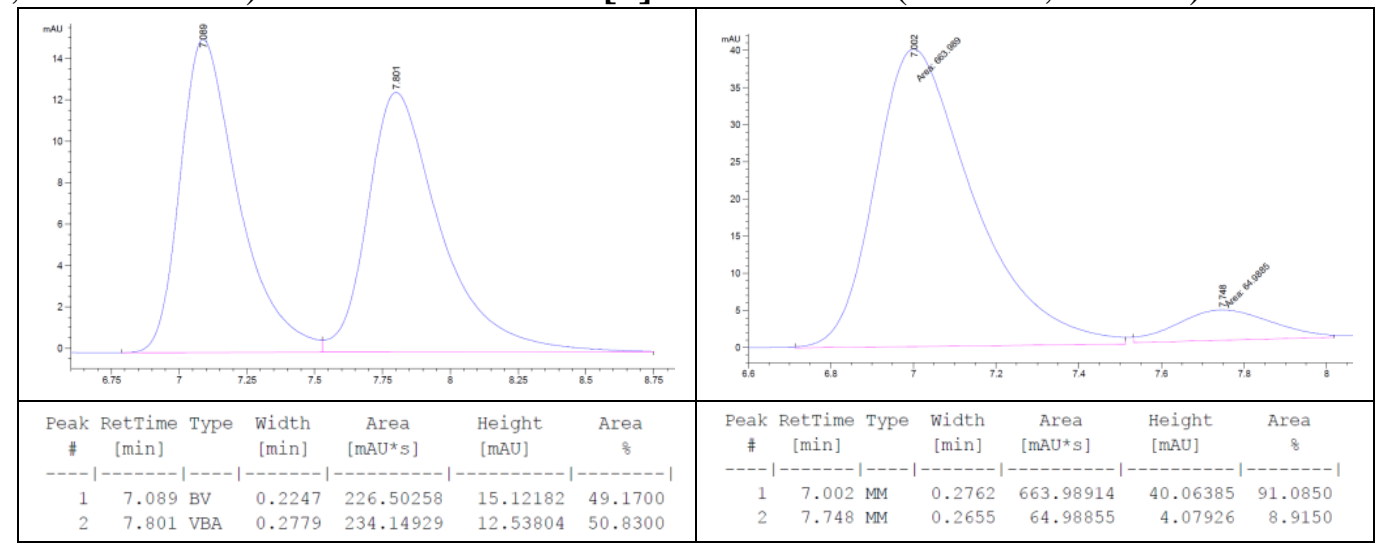

\section{(S)-1-(Phenylethynyl)spiro[2.5]octane (2f)}

Alkynylated cyclopropane 2f was prepared according to the general procedure. After standard work-up and purification, $2 \mathbf{f}$ was obtained as a colorless oil $(18.9 \mathrm{mg}, 45 \%$ yield). 
$1 \mathrm{H}$ NMR (400 MHz, CDCl3) $\delta 7.30(\mathrm{dd}, J=7.5,2.1 \mathrm{~Hz}, 2 \mathrm{H}), 7.23-7.11(\mathrm{~m}, 3 \mathrm{H}), 1.64$ $-1.25(\mathrm{~m}, 9 \mathrm{H}), 1.25-1.10(\mathrm{~m}, 2 \mathrm{H}), 0.74(\mathrm{dd}, J=8.4,4.1 \mathrm{~Hz}, 1 \mathrm{H}), 0.56(\mathrm{t}, J=4.6 \mathrm{~Hz}$, 1H). 13C NMR (100 MHz, $\left.\mathrm{CDCl}_{3}\right) \delta 131.5,128.1,127.2,124.3,91.5,77.8,36.2,32.1$, 27.1, 26.0, 25.5, 25.3, 22.4, 13.4. HRMS (TOF-MS ES+): [M+H]+, calculated for $\mathrm{C}_{16 \mathrm{H} 19:}$ 211.1481; found 211.1418.

Chiral HPLC analysis (CHIRALCEL IA, 100\% hexane, $1.0 \mathrm{~mL} / \mathrm{min}, 254 \mathrm{~nm}$, tR1 $=4.7$ min, $\left.\mathrm{tR}_{2}=5.0 \mathrm{~min}\right)$ indicated 95:05 er. $[\alpha] \mathrm{D} 20=+127.08\left(\mathrm{c}=0.67, \mathrm{CH}_{2} \mathrm{Cl}_{2}\right)$.

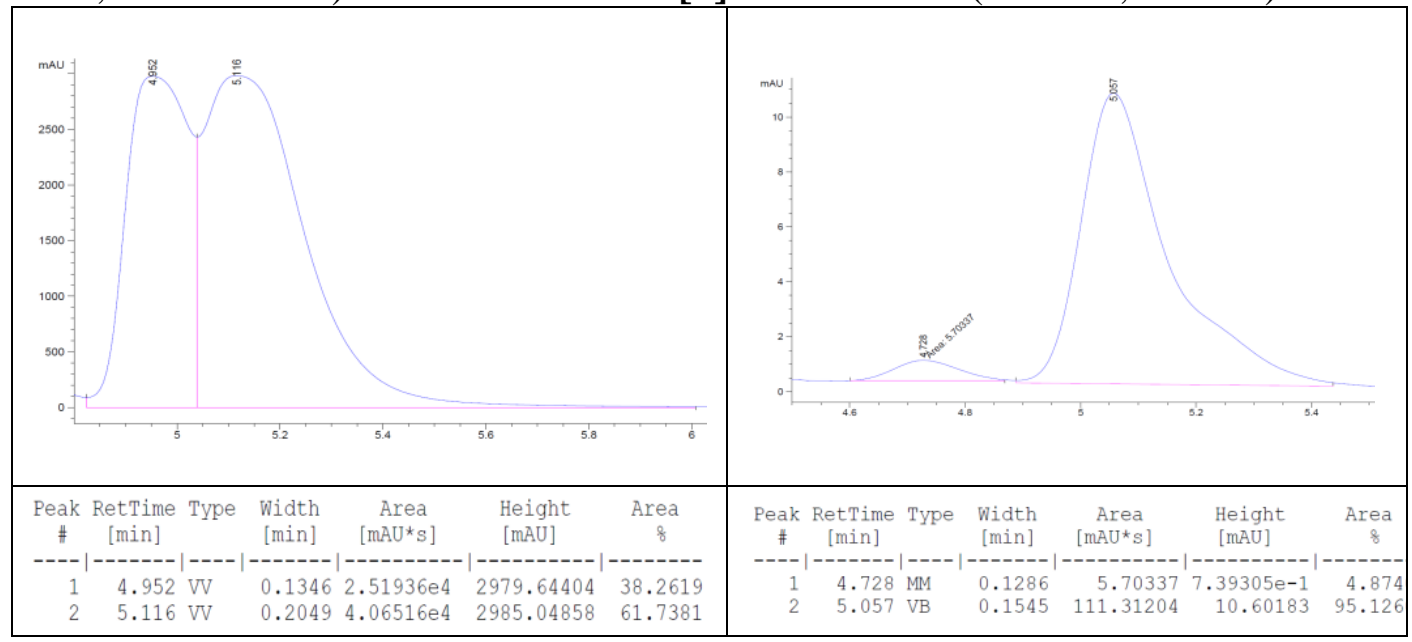

\section{(S)-((2,2-Dimethylcyclopropyl)ethynyl)benzene (2g)}

Alkynylated cyclopropane $\mathbf{2 g}$ was prepared according to the general procedure. After standard work-up and purification, $\mathbf{2 g}$ was obtained as a colorless oil $(16.7 \mathrm{mg}, 49 \%$ yield).

1 H NMR (400 MHz, CDCl 3$) \delta 7.33-7.26(\mathrm{~m}, 2 \mathrm{H}), 7.23-7.16(\mathrm{~m}, 3 \mathrm{H}), 1.25(\mathrm{dd}, J=$ 8.6, $5.2 \mathrm{~Hz}, 1 \mathrm{H}), 1.21(\mathrm{~s}, 3 \mathrm{H}), 1.06(\mathrm{~s}, 3 \mathrm{H}), 0.78(\mathrm{dd}, J=8.6,4.1 \mathrm{~Hz}, 1 \mathrm{H}), 0.57(\mathrm{t}, J=4.7$ $\mathrm{Hz}, 1 \mathrm{H}) .13 \mathrm{C} \mathrm{NMR}\left(100 \mathrm{MHz}, \mathrm{CDCl}_{3}\right) \delta 131.5,128.1,127.3,124.2,91.5,78.2,25.7$, 23.5, 21.5, 19.9, 14.5. HRMS (TOF-MS ES+): $[\mathbf{M + H}]_{+}$, calculated for $\mathrm{C}_{13} \mathrm{H}_{15}$ : 171.1168; found 171.1137.

Chiral HPLC (CHIRALCEL IA, 100\% hexane, $1.0 \mathrm{~mL} / \mathrm{min}, 254 \mathrm{~nm}, \mathrm{tR} 1=4.4 \mathrm{~min}$, tR2 $=5.0 \mathrm{~min})$ indicated $88: 12 \mathrm{er} .[\alpha]_{\mathbf{D} 20}=+95.67\left(\mathrm{c}=0.18, \mathrm{CH}_{2} \mathrm{Cl}_{2}\right)$.

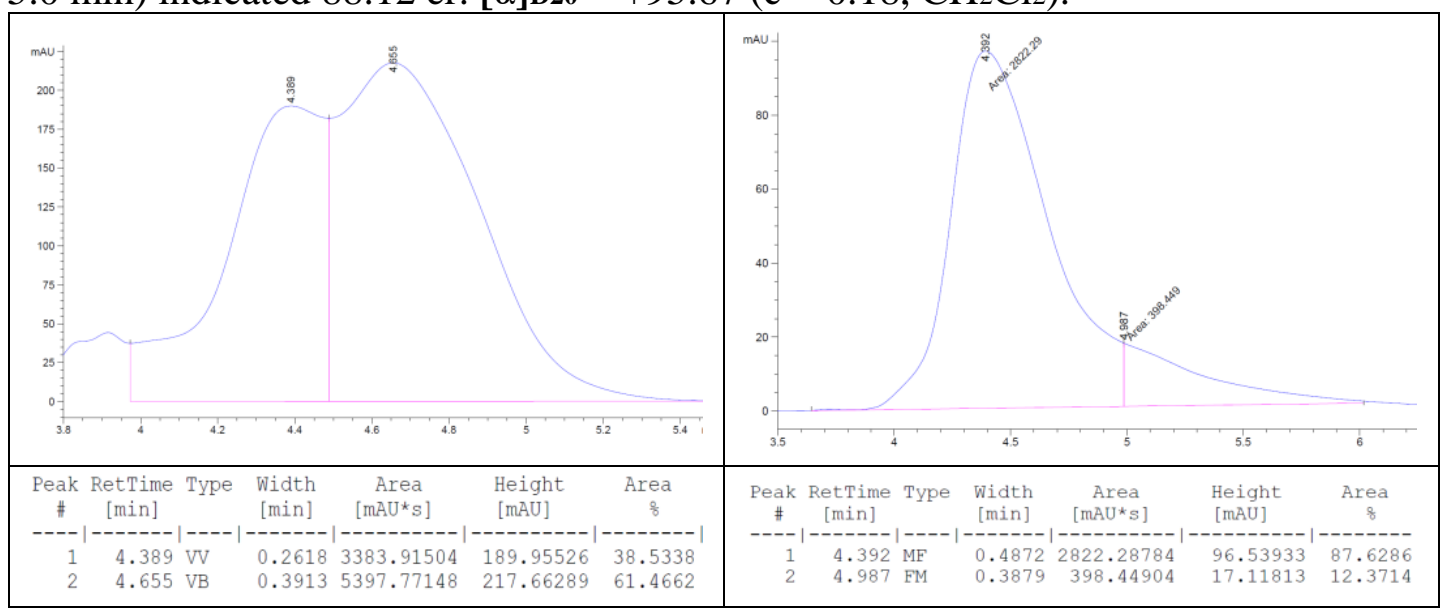




\section{1-Methyl-3-(((1S,2S)-2-methyl-2-phenylcyclopropyl)ethynyl)benzene (2h)}

Alkynylated cyclopropane $\mathbf{2 h}$ was prepared according to the general procedure. After standard work-up and purification, $\mathbf{2 h}$ was obtained as a colorless oil $(30.0 \mathrm{mg}, 61 \%$ yield).

1H NMR (400 MHz, CDCl3) $\delta$ 7.13-7.10 (m, 4H), 7.04-6.99 (m, 3H), 6.91-6.88 (m, 1H), $2.13(\mathrm{~s}, 3 \mathrm{H}), 1.63(\mathrm{dd}, J=5.6,8.9 \mathrm{~Hz}, 1 \mathrm{H}), 1.43(\mathrm{~s}, 3 \mathrm{H}), 1.29$ (dd, $J=4.5,9.0 \mathrm{~Hz}, 1 \mathrm{H})$, 0.84 (dd, $J=4.6,5.5 \mathrm{~Hz}, 1 \mathrm{H})$. $13 \mathrm{C}$ NMR (100 MHz, CDCl $)$ 145.7, 137.8, 132.2, 128.7 , 128.4, 128.4, 128.1, 127.3, 126.2, 123.7, 90.0, 27.9, 23.3, 22.6, 21.2, 16.0. HRMS (TOFMS ES+): [M+H]+, calculated for $\mathrm{C}_{19} \mathrm{H}_{19}$ : 247.1481; found 247.1464.

Chiral HPLC (CHIRALCEL IA, 100\% hexane, $1.0 \mathrm{~mL} / \mathrm{min}, 254 \mathrm{~nm}, \mathrm{tR} 1=8.5 \mathrm{~min}$, tR2 $=9.6 \mathrm{~min})$ indicated $90: 10 \mathrm{er} .[\alpha]_{\mathbf{D} 20}=+278.53\left(\mathrm{c}=0.13, \mathrm{CH}_{2} \mathrm{Cl}_{2}\right)$.

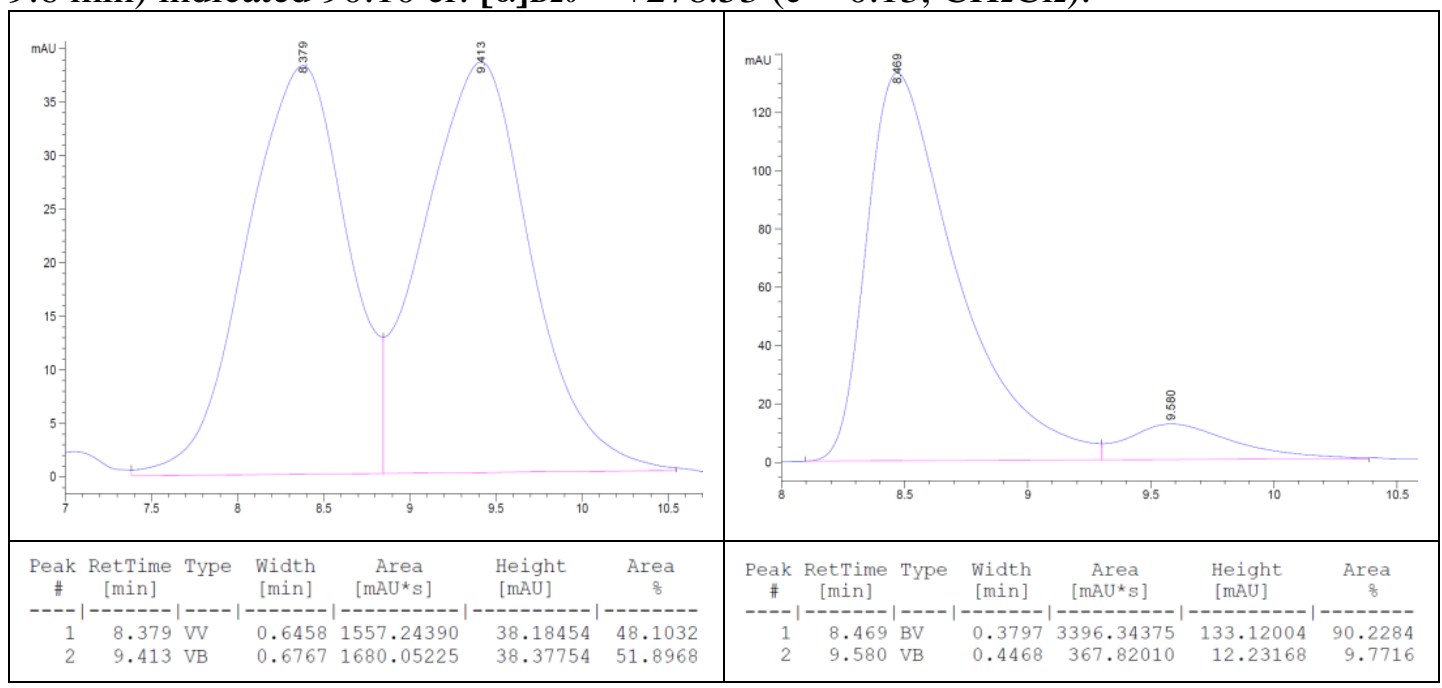

\section{1-(((1S,2S)-2-Methyl-2-phenylcyclopropyl)ethynyl)-4-propylbenzene (2i)}

Alkynylated cyclopropane 2i was prepared according to the general procedure 1. After standard work-up and purification, $\mathbf{2 i}$ was obtained as a colorless oil $(37.8 \mathrm{mg}, 69 \%$ yield).

1H NMR (400 MHz, CDCl 3$) \delta 7.31-7.20(\mathrm{~m}, 8 \mathrm{H}), 7.16-7.08(\mathrm{~m}, 1 \mathrm{H}), 1.75(\mathrm{dd}, J=$ $8.9,5.7 \mathrm{~Hz}, 1 \mathrm{H}), 1.54(\mathrm{~s}, 3 \mathrm{H}), 1.40(\mathrm{dd}, J=8.9,4.6 \mathrm{~Hz}, 1 \mathrm{H}), 1.23(\mathrm{~s}, 9 \mathrm{H}), 0.95$ (dd, $J=$ $5.7,4.5 \mathrm{~Hz}, 1 \mathrm{H}) .13 \mathrm{C}$ NMR (100 MHz, CDCl3) $\delta 145.7,142.3,131.48,128.4,127.2$, $126.2,121.1,89.5,79.4,37.9,27.8,24.4,23.3,22.5,16.1,13.7$.

HRMS (TOF-MS ES+): [M+H]+, calculated for $\mathrm{C}_{21} \mathrm{H}_{23}$ : 275.1794; found 275.1720.

Chiral HPLC (CHIRALCEL IA, 100\% hexane, $1.0 \mathrm{~mL} / \mathrm{min}, 254 \mathrm{~nm}, \mathrm{tR} 1=8.0 \mathrm{~min}$, tR2 $=8.9 \mathrm{~min})$ indicated $88: 12 \mathrm{er} .[\alpha]_{\mathbf{D} 20}=+224.74\left(\mathrm{c}=0.86, \mathrm{CH}_{2} \mathrm{Cl}_{2}\right)$. 


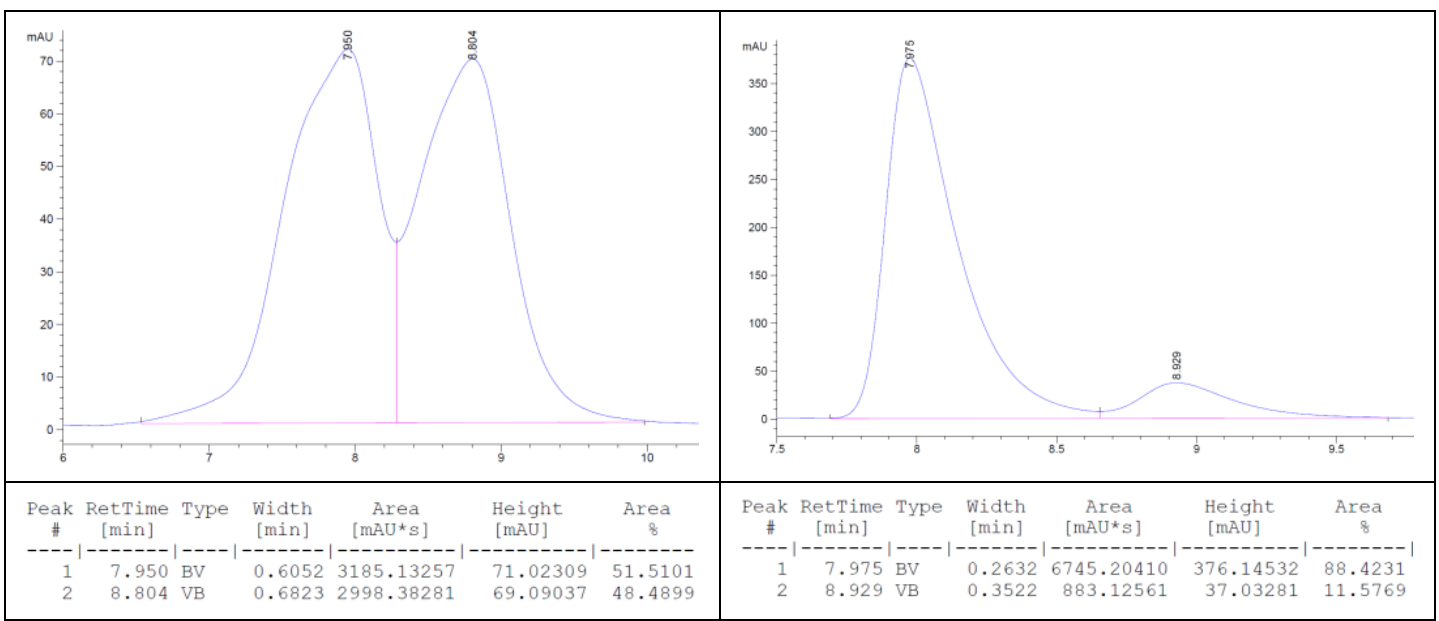

\section{1-Tert-butyl-4-(((1S,2S)-2-methyl-2-phenylcyclopropyl)ethynyl)benzene} $(2 \mathbf{j})$

Alkynylated cyclopropane $\mathbf{2} \mathbf{j}$ was prepared according to the general procedure. After standard work-up and purification, $\mathbf{2} \mathbf{j}$ was obtained as a colorless oil $(31.7 \mathrm{mg}, 55 \%$ yield).

1H NMR (400 MHz, CDCl3) $\delta 7.31-7.20$ (m, 8H), $7.16-7.08(\mathrm{~m}, 1 \mathrm{H}), 1.75$ (dd, $J=$ 8.9, $5.7 \mathrm{~Hz}, 1 \mathrm{H}), 1.54(\mathrm{~s}, 3 \mathrm{H}), 1.40$ (dd, $J=8.9,4.6 \mathrm{~Hz}, 1 \mathrm{H}), 1.23$ (s, 9H), 0.95 (dd, $J=$ 5.7, $4.5 \mathrm{~Hz}, 1 \mathrm{H}) .13 \mathrm{C}$ NMR (100 MHz, CDCl3) $\delta$ 150.7, 145.7, 131.3, 128.4, 127.2, 126.2, 125.2, 120.9, 89.6, 79.3, 34.7, 31.2, 27.8, 23.3, 22.5, 16.1. HRMS (TOF-MS $\left.\mathbf{E S}_{+}\right)$: $[\mathbf{M + H}]_{+}$, calculated for $\mathrm{C}_{22} \mathrm{H}_{25}$ : 289.1951; found 289.1928 .

Chiral HPLC (CHIRALCEL IA, 100\% hexane, $1.0 \mathrm{~mL} / \mathrm{min}, 254 \mathrm{~nm}$, tR1 = $8.2 \mathrm{~min}$, tR2 $=8.9 \mathrm{~min})$ indicated 93:07 er. $[\alpha] \mathbf{D 2 0}=+212.88\left(\mathrm{c}=0.73, \mathrm{CH}_{2} \mathrm{Cl}_{2}\right)$.

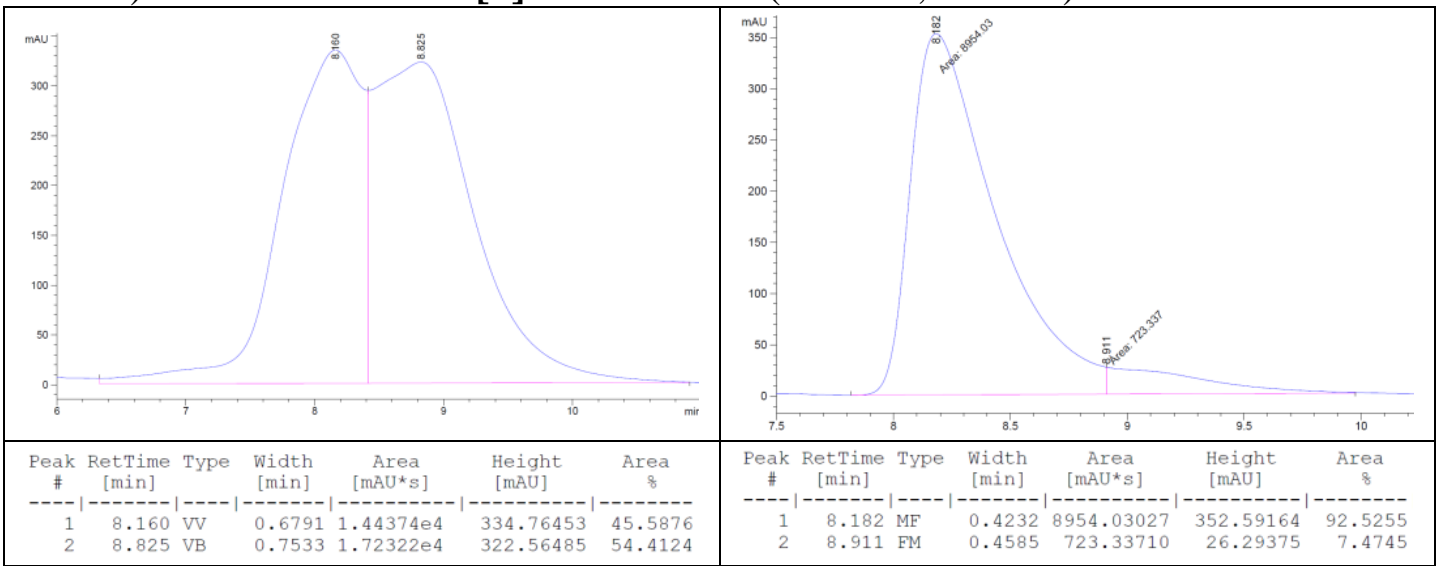

\section{1-Methoxy-4-(((1S,2S)-2-methyl-2-phenylcyclopropyl)ethynyl)benzene}

\section{$(2 \mathbf{k})$}

Alkynylated cyclopropane $\mathbf{2 k}$ was prepared according to the general procedure. After standard work-up and purification, $\mathbf{2 k}$ was obtained as a colorless oil $(39.3 \mathrm{mg}, 75 \%$ yield). 
1H NMR (400 MHz, CDCl3) $\delta 7.24-7.19(\mathrm{~m}, 2 \mathrm{H}), 7.18-7.11(\mathrm{~m}, 4 \mathrm{H}), 7.05$ (ddd, $J=$ 8.5, 7.1, 4.3 Hz, 1H), $6.67(\mathrm{~d}, J=8.7 \mathrm{~Hz}, 2 \mathrm{H}), 3.64(\mathrm{~s}, 3 \mathrm{H}), 1.67(\mathrm{dd}, J=8.9,5.7 \mathrm{~Hz}$, $1 \mathrm{H}), 1.47(\mathrm{~s}, 3 \mathrm{H}), 1.32(\mathrm{dd}, J=8.9,4.5 \mathrm{~Hz}, 1 \mathrm{H}), 0.87(\mathrm{dd}, J=5.6,4.5 \mathrm{~Hz}, 1 \mathrm{H}) .13 \mathrm{C} \mathrm{NMR}$ (100 MHz, CDCl3) $\delta 159.0,145.7,133.0,128.4,128.3,127.2,126.2,116.1,113.8,88.7$, 79.1, 55.2, 27.7, 23.2, 22.5, 16.1.

Chiral HPLC (CHIRALCEL IA, 100\% hexane, $1.0 \mathrm{~mL} / \mathrm{min}, 254 \mathrm{~nm}$, tR1 = $21.2 \mathrm{~min}$, tR2 $=26.2 \mathrm{~min})$ indicated 96:04 er. $[\alpha] \mathbf{D 2 0}=+272.36\left(\mathrm{c}=0.50, \mathrm{CH}_{2} \mathrm{Cl}_{2}\right),[\alpha]_{\mathbf{D} 20}=+375.36(\mathrm{c}$ $\left.=0.30, \mathrm{CHCl}_{3}\right)$, while the literuate reported $(R, R)-2 \mathbf{k}[\alpha]_{\mathrm{D} 25}=-460.0\left(\mathrm{c}=2, \mathrm{CHCl}_{3}\right)$, $99 \%$ ee. 2

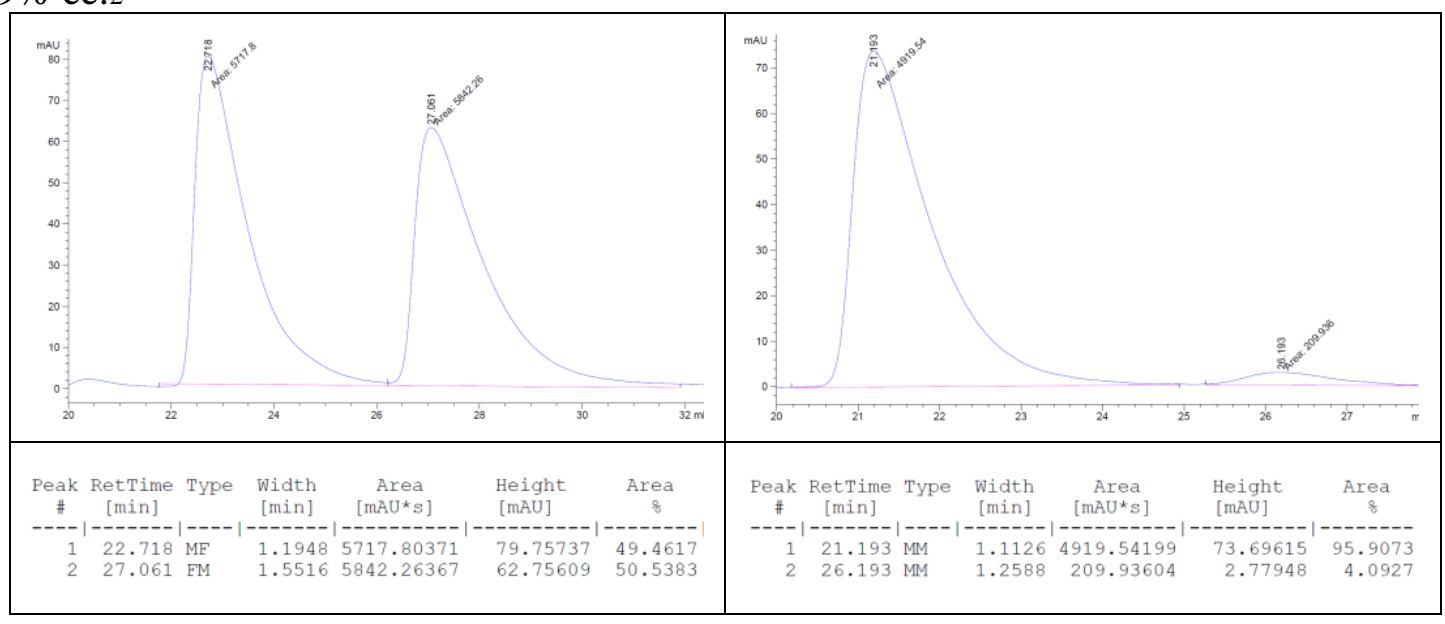

\section{1,2-Dimethoxy-4-(((1S,2S)-2-methyl-2- phenylcyclopropyl)ethynyl)benzene (2l)}

Alkynylated cyclopropane 2l was prepared according to the general procedure. After standard work-up and purification, 21 was obtained as a colorless oil (42.7 $\mathrm{mg}, 73 \%$ yield).

1H NMR $\delta 7.28-7.20(\mathrm{~m}, 4 \mathrm{H}), 7.13(\mathrm{dd}, J=3.3,2.5 \mathrm{~Hz}, 1 \mathrm{H}), 6.96(\mathrm{dd}, J=8.3,1.9 \mathrm{~Hz}$, $1 \mathrm{H}), 6.87(\mathrm{~d}, J=1.8 \mathrm{~Hz}, 1 \mathrm{H}), 6.71(\mathrm{~d}, J=8.3 \mathrm{~Hz}, 1 \mathrm{H}), 3.80(\mathrm{~s}, 6 \mathrm{H}), 1.74(\mathrm{dd}, J=8.9,5.7$ $\mathrm{Hz}, 1 \mathrm{H}), 1.55$ (s, 3H), $1.40(\mathrm{dd}, J=8.9,4.5 \mathrm{~Hz}, 1 \mathrm{H}), 0.95(\mathrm{dd}, J=5.6,4.6 \mathrm{~Hz}, 1 \mathrm{H}) .13 \mathrm{C}$ NMR $\left(100 \mathrm{MHz}, \mathrm{CDCl}_{3}\right) \delta 148.9,148.5,145.7,128.4,127.2,126.2,124.7,116.2,114.4$, 110.9, 88.6, 79.2, 55.8, 27.8, 23.2, 22.6, 16.0. HRMS (TOF-MS ES+): [M+H]+, calculated for $\mathrm{C}_{20} \mathrm{H}_{21} \mathrm{O}$ : 293.1536; found 293.1547.

Chiral HPLC (CHIRALCEL IA, 98\% hexane/2\%IPA, $1.0 \mathrm{~mL} / \mathrm{min}, 254 \mathrm{~nm}$, tR1 = 8.5 $\left.\min , \mathrm{tR}_{2}=10.7 \mathrm{~min}\right)$ indicated 96:04 er. $[\alpha]_{\mathbf{D} 20}=+265.71\left(\mathrm{c}=0.645, \mathrm{CH}_{2} \mathrm{Cl}_{2}\right)$. 


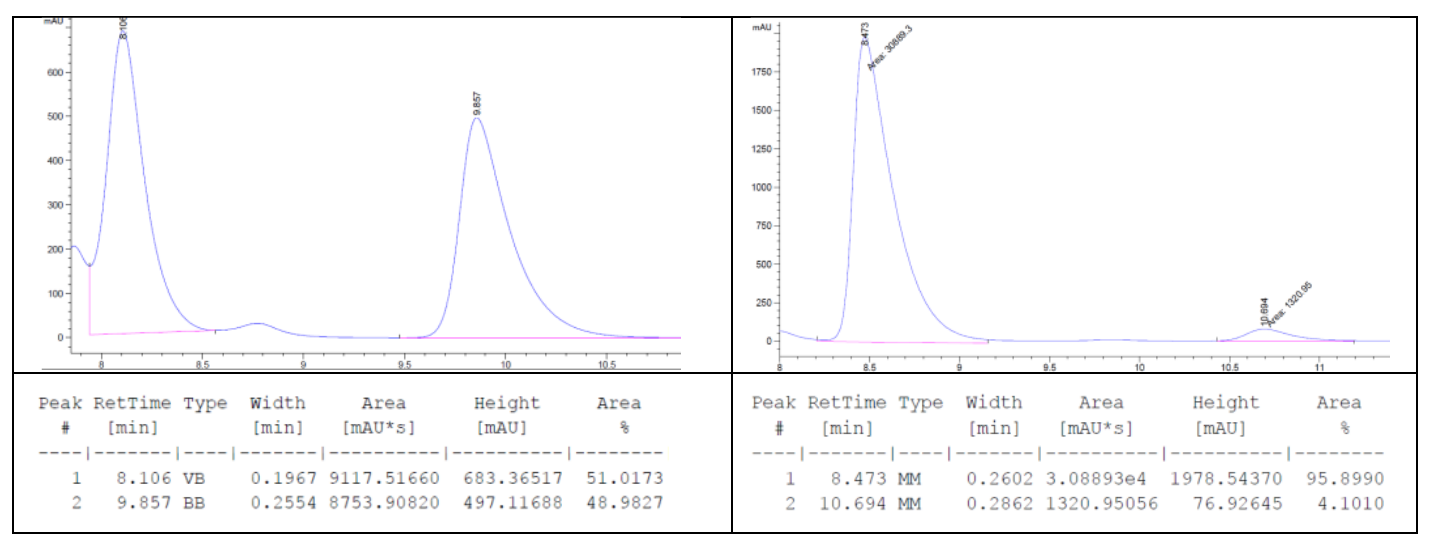

\section{1-Bromo-4-(((1S,2S)-2-methyl-2-phenylcyclopropyl)ethynyl)benzene (2m)}

Alkynylated cyclopropane $\mathbf{2 m}$ was prepared according to the general procedure. After standard work-up and purification, $\mathbf{2 m}$ was obtained as a colorless oil $(49.2 \mathrm{mg}, 79 \%$ yield).

1H NMR (600 MHz, CDCl 3$) \delta 7.49-7.42(\mathrm{~m}, 2 \mathrm{H}), 7.38-7.29(\mathrm{~m}, 6 \mathrm{H}), 7.24(\mathrm{p}, J=4.4$ $\mathrm{Hz}, 1 \mathrm{H}), 1.84(\mathrm{dd}, J=8.9,5.7 \mathrm{~Hz}, 1 \mathrm{H}), 1.64(\mathrm{~s}, 3 \mathrm{H}), 1.53(\mathrm{dd}, J=8.9,4.5 \mathrm{~Hz}, 1 \mathrm{H}), 1.08$ $(\mathrm{dd}, J=5.7,4.5 \mathrm{~Hz}, 1 \mathrm{H}) .{ }_{13} \mathrm{C} \mathbf{N M R}\left(150 \mathrm{MHz}, \mathrm{CDCl}_{3}\right) \delta 145.4,133.1,131.4,128.4$, 127.3, 126.3, 122.9, 121.6, 91.7, 78.3, 28.1, 23.2, 22.6, 15.9.

HRMS (TOF-MS ES+): [M+H] + , calculated for $\mathrm{C}_{18} \mathrm{H}_{16} \mathrm{Br}: 311.0430$; found 311.0452 .

Chiral HPLC (CHIRALCEL IA, 100\% hexane, $1.0 \mathrm{~mL} / \mathrm{min}, 254 \mathrm{~nm}$, tR1 $=9.7 \mathrm{~min}$, tR2 $=11.2 \mathrm{~min})$ indicated 96:04 er. $[\alpha]_{\mathbf{D} 20}=+286.35\left(\mathrm{c}=0.51, \mathrm{CH}_{2} \mathrm{Cl}_{2}\right)$.

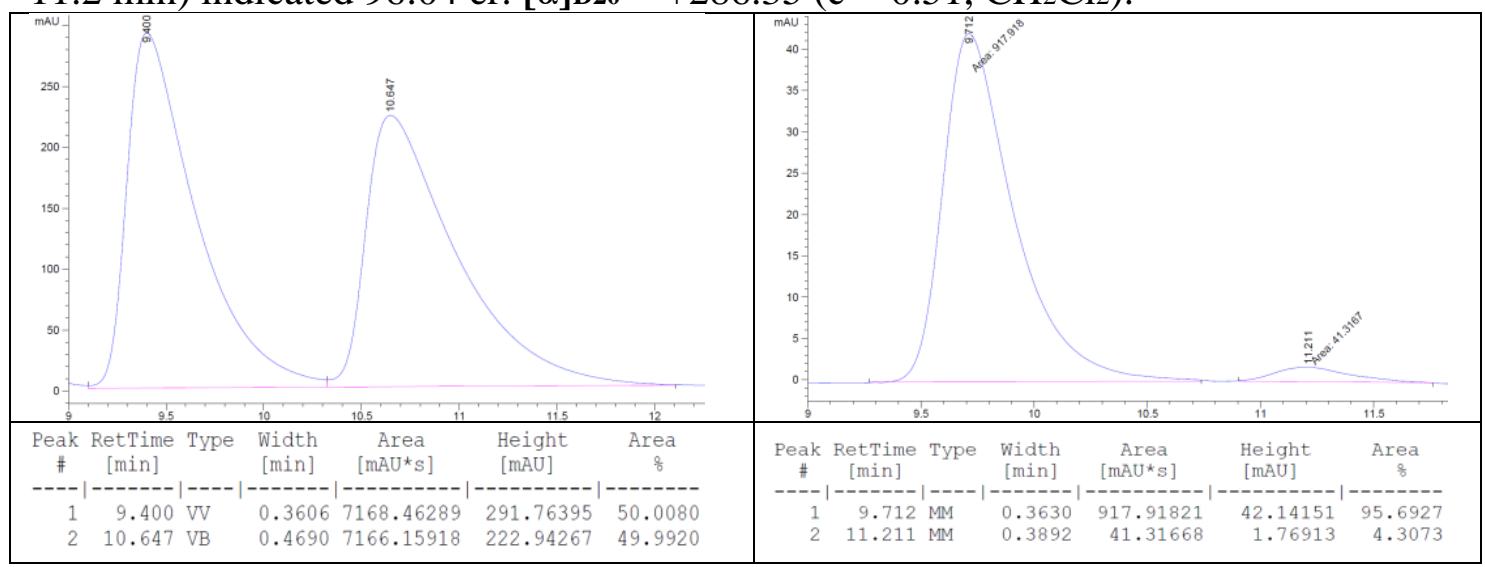

\section{1-Fluoro-4-(((1S,2S)-2-methyl-2-phenylcyclopropyl)ethynyl)benzene (2n)}

Alkynylated cyclopropane $\mathbf{2 n}$ was prepared according to the general procedure. After standard work-up and purification, $2 \mathrm{n}$ was obtained as a colorless oil $(30.5 \mathrm{mg}, 61 \%$ yield).

1H NMR (400 MHz, CDCl3) $\delta$ 7.23-7.19 (m, 2H), 7.13-7.10 (m, 4H), 7.04-7.00 (m, 2H), $6.80(\mathrm{t}, J=8.8 \mathrm{~Hz}, 2 \mathrm{H}), 1.62(\mathrm{dd}, J=5.7,8.9 \mathrm{~Hz}, 1 \mathrm{H}), 1.42(\mathrm{~s}, 3 \mathrm{H}), 1.30(\mathrm{dd}, J=4.5,8.9$ $\mathrm{Hz}, 1 \mathrm{H}), 0.84(\mathrm{dd}, J=4.5,5.7 \mathrm{~Hz}, 1 \mathrm{H}) .13 \mathrm{C}$ NMR (100 MHz, CDCl3) $\delta 162.0$ (d, $J=$ $246.5 \mathrm{~Hz}$ ), 145.6, 133.4 (d, $J=8.2 \mathrm{~Hz}), 128.4,127.3,126.3,120.0$ (d, $J=3.4 \mathrm{~Hz}), 115.4$ 
(d, $J=\mathrm{Hz}$ ), 90.0, 78.2, 27.9, 23.2, 22.6, 15.9. HRMS (TOF-MS ES+): [M+H]+, calculated for $\mathrm{C}_{18} \mathrm{H}_{16} \mathrm{~F}: 251.1231$; found 251.1207.

Chiral HPLC (CHIRALCEL IA, 100\% hexane, $1.0 \mathrm{~mL} / \mathrm{min}, 254 \mathrm{~nm}, \mathrm{tR} 1=7.8 \mathrm{~min}$, tR2 $=8.6 \mathrm{~min})$ indicated 97:03 er. $[\alpha]_{\mathbf{D} 20}=+225.62\left(\mathrm{c}=0.23, \mathrm{CH}_{2} \mathrm{Cl}_{2}\right)$.

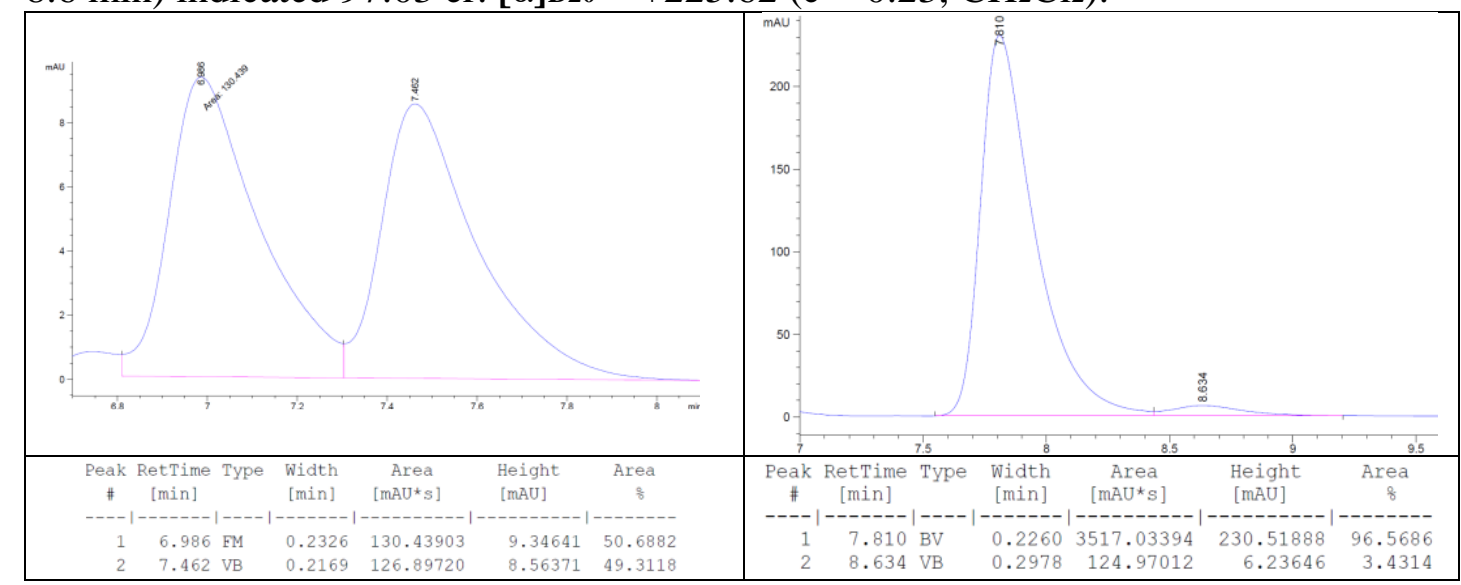

\section{1-Fluoro-3-(((1S,2S)-2-methyl-2-phenylcyclopropyl)ethynyl)benzene (2o)}

Alkynylated cyclopropane 20 was prepared according to the general procedure. After standard work-up and purification, 20 was obtained as a colorless oil $(26.5 \mathrm{mg}, 53 \%$ yield).

1H NMR $\left(600 \mathrm{MHz}, \mathrm{CDCl}_{3}\right) \delta 7.37-7.20(\mathrm{~m}, 7 \mathrm{H}), 7.15(\mathrm{ddd}, J=9.6,2.7,1.5 \mathrm{~Hz}, 1 \mathrm{H})$, 7.01 (tdd, $J=8.4,2.7,1.1 \mathrm{~Hz}, 1 \mathrm{H}), 1.85(\mathrm{dd}, J=8.9,5.6 \mathrm{~Hz}, 1 \mathrm{H}), 1.65$ (s, $3 \mathrm{H}), 1.53$ (dd, $J=8.9,4.5 \mathrm{~Hz}, 1 \mathrm{H}), 1.08(\mathrm{dd}, J=5.7,4.5 \mathrm{~Hz}, 1 \mathrm{H}) .13 \mathrm{C} \mathrm{NMR}\left(150 \mathrm{MHz}, \mathrm{CDCl}_{3}\right) \delta 162.4$ $(\mathrm{d}, J=244.5 \mathrm{~Hz}), 145.5,129.8(d, \mathrm{~J}=7.5 \mathrm{~Hz}), 128.5,127.5(\mathrm{~d}, J=3.0 \mathrm{~Hz}), 127.3,126.4$, $125.8(\mathrm{~d}, J=9.6 \mathrm{~Hz}), 118.4(\mathrm{~d}, J=9.6 \mathrm{~Hz}), 114.9(\mathrm{~d}, J=20.9 \mathrm{~Hz}), 91.6,78.3(\mathrm{~d}, J=3.3$ $\mathrm{Hz}), 28.2,23.3,22.7,15.9$.

HRMS (TOF-MS ES+): [M+H]+, calculated for $\mathrm{C}_{18} \mathrm{H}_{16} \mathrm{~F}: 251.1231$; found 251.1211.

Chiral HPLC (CHIRALCEL IA, 100\% hexane, $1.0 \mathrm{~mL} / \mathrm{min}, 254 \mathrm{~nm}, \mathrm{tR} 1=8.8 \mathrm{~min}$, tR2 $=10.4 \mathrm{~min})$ indicated 93:07 er. $[\alpha] \mathbf{D 2 0}=+226.82\left(\mathrm{c}=0.30, \mathrm{CH}_{2} \mathrm{Cl}_{2}\right)$.

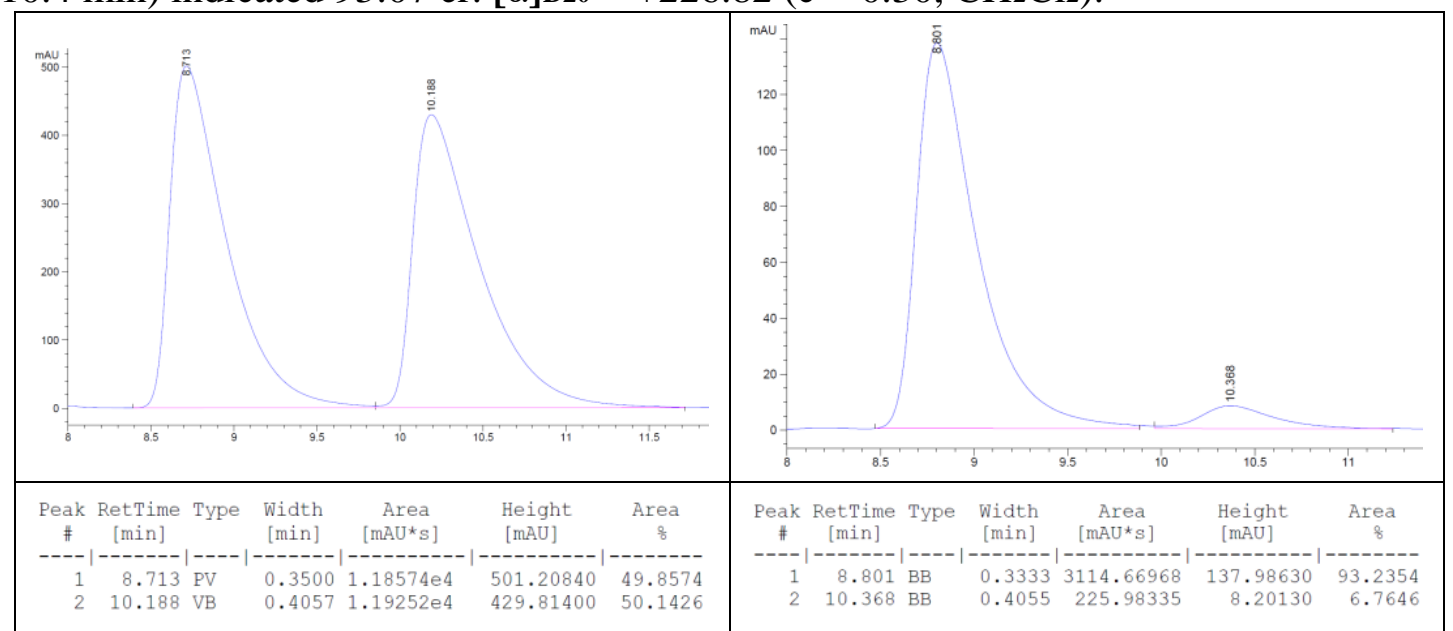

1-Fluoro-2-(((1S,2S)-2-methyl-2-phenylcyclopropyl)ethynyl)benzene (2p) 
Alkynylated cyclopropane 2p was prepared according to the general procedure. After standard work-up and purification, $\mathbf{2 p}$ was obtained as a colorless oil $(24.5 \mathrm{mg}, 49 \%$ yield).

1H NMR $\left(600 \mathrm{MHz}, \mathrm{CDCl}_{3}\right) \delta 7.45(\mathrm{td}, J=7.5,2.0 \mathrm{~Hz}, 1 \mathrm{H}), 7.37-7.31(\mathrm{~m}, 4 \mathrm{H}), 7.28-$ $7.22(\mathrm{~m}, 2 \mathrm{H}), 7.15-7.04(\mathrm{~m}, 2 \mathrm{H}), 1.91(\mathrm{~s}, 1 \mathrm{H}), 1.67(\mathrm{~s}, 3 \mathrm{H}), 1.55(\mathrm{dd}, J=8.9,4.6 \mathrm{~Hz}$, $1 \mathrm{H}), 1.12(\mathrm{dd}, J=5.6,4.5 \mathrm{~Hz}, 1 \mathrm{H}), 13 \mathrm{C}$ NMR $\left(150 \mathrm{MHz}, \mathrm{CDCl}_{3}\right) \delta 162.9(\mathrm{~d}, J=248.6$ $\mathrm{Hz}), 145.48,133.5,129.0(\mathrm{~d}, J=7.0 \mathrm{~Hz}), 128.4,127.3,126.3,123.8(\mathrm{~d}, J=3.8 \mathrm{~Hz})$, $115.3(\mathrm{~d}, J=21.0 \mathrm{~Hz}), 112.4(\mathrm{~d}, J=15.8 \mathrm{~Hz}), 95.9(\mathrm{~d}, J=3.3 \mathrm{~Hz}), 72.5,28.2,23.4,22.6$, 16.1 .

HRMS (TOF-MS ES+): [M+H]+, calculated for $\mathrm{C}_{18} \mathrm{H}_{16} \mathrm{~F}$ : 251.1231; found 251.1205.

Chiral HPLC (CHIRALCEL IA, 100\% hexane, $1.0 \mathrm{~mL} / \mathrm{min}, 254 \mathrm{~nm}, \mathrm{tR} 1=8.2 \mathrm{~min}$, tR2 $=9.4 \mathrm{~min})$ indicated 93:07 er. $[\alpha]_{\mathbf{D} 20}=+223.35\left(\mathrm{c}=0.15, \mathrm{CH}_{2} \mathrm{Cl}_{2}\right)$.

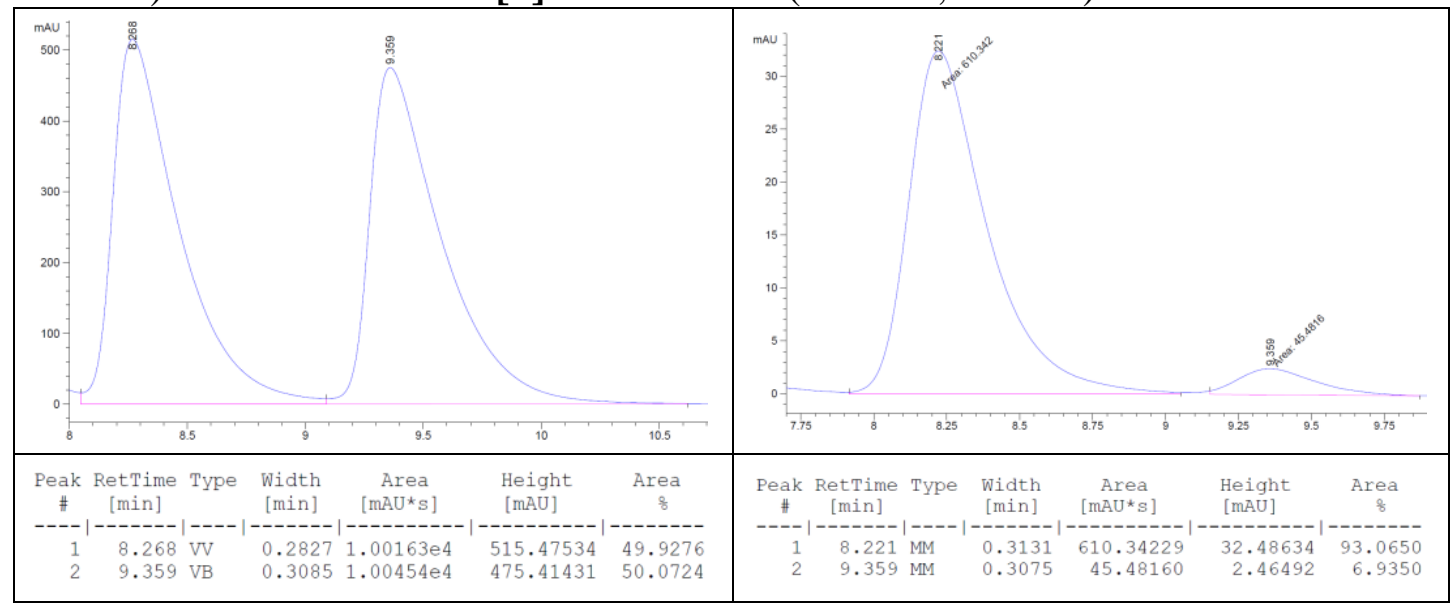

\section{1-Chloro-4-((1S,2S)-2-((4-fluorophenyl)ethynyl)-1- methylcyclopropyl)benzene (2q)}

Alkynylated cyclopropane $\mathbf{2 q}$ was prepared according to the general procedure. After standard work-up and purification, $\mathbf{2 q}$ was obtained as a colorless oil $(31.3 \mathrm{mg}, 55 \%$ yield).

1H NMR (400 MHz, CDCl) $\delta 7.36-7.29(\mathrm{~m}, 2 \mathrm{H}), 7.22-7.10(\mathrm{~m}, J=7.9 \mathrm{~Hz}, 4 \mathrm{H}), 6.94$ $-6.89(\mathrm{~m}, 2 \mathrm{H}), 1.70(\mathrm{dd}, J=9.0,5.7 \mathrm{~Hz}, 1 \mathrm{H}), 1.52(\mathrm{~s}, 3 \mathrm{H}), 1.37(\mathrm{dd}, J=9.0,4.7 \mathrm{~Hz}$, 1H), $0.99-0.94(\mathrm{~m}, 1 \mathrm{H}) . \mathbf{1 3 C}$ NMR (100 MHz, CDCl $) \delta 163.3,160.8,144.1,133.4(\mathrm{~d}$, $J=30.8 \mathrm{~Hz}), 132.0,128.6(\mathrm{~d}, J=72.4 \mathrm{~Hz}), 119.9(\mathrm{~d}, J=25.3 \mathrm{~Hz}), 115.4(\mathrm{~d}, J=86.5$ $\mathrm{Hz}), 89.49,78.50,27.4,23.2,22.5,16.0$.

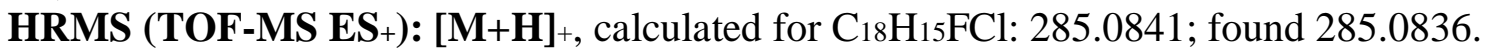
Chiral HPLC (CHIRALCEL IA, 100\% hexane, $1.0 \mathrm{~mL} / \mathrm{min}, 254 \mathrm{~nm}, \mathrm{tR} 1=9.0 \mathrm{~min}$, tR2 $=10.8 \mathrm{~min})$ indicated 96:04 er. $[\alpha] \mathbf{D 2 0}=+267.90\left(\mathrm{c}=0.37, \mathrm{CH}_{2} \mathrm{Cl}_{2}\right)$. 


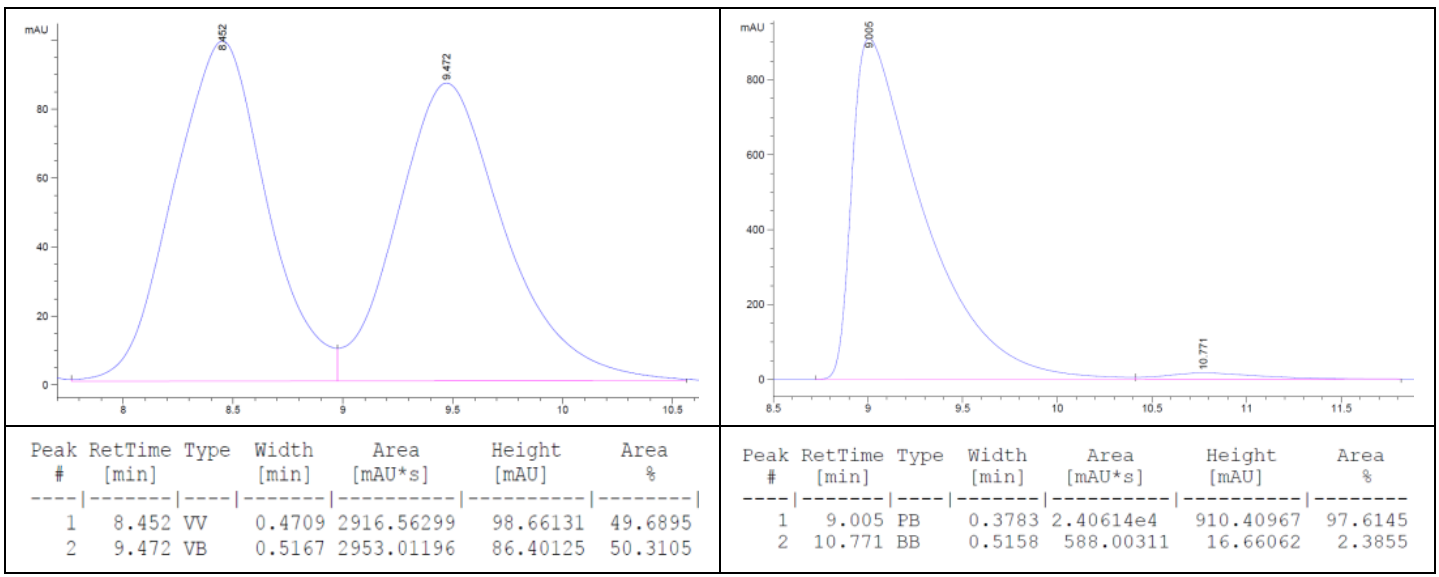

\section{1-Chloro-4-((1S,2S)-2-((4-methoxyphenyl)ethynyl)-1- methylcyclopropyl)benzene (2r)}

Alkynylated cyclopropane $\mathbf{2 r}$ was prepared according to the general procedure. After standard work-up and purification, $\mathbf{2 r}$ was obtained as a colorless oil $(39.8 \mathrm{mg}, 67 \%$ yield).

1H NMR (400 MHz, CDCl3) $\delta 7.28(\mathrm{~d}, J=8.8 \mathrm{~Hz}, 2 \mathrm{H}), 7.19-7.12(\mathrm{~m}, 4 \mathrm{H}), 6.74(\mathrm{~d}, J=$ $8.8 \mathrm{~Hz}, 2 \mathrm{H}), 3.71(\mathrm{~s}, 3 \mathrm{H}), 1.69(\mathrm{dd}, J=5.7,8.9 \mathrm{~Hz}, 1 \mathrm{H}), 1.50(\mathrm{~s}, 3 \mathrm{H}), 1.34$ (dd, $J=4.6$, $9.0 \mathrm{~Hz}, 1 \mathrm{H}), 0.94(\mathrm{dd}, J=4.8,4.80 \mathrm{~Hz}, 1 \mathrm{H}) .{ }_{13} \mathrm{C}$ NMR $\left(100 \mathrm{MHz}, \mathrm{CDCl}_{3}\right) \delta 159.1$, 144.3, 133.0, 131.9, 128.6, 128.4, 115.9, 113.8, 88.2, 79.3, 55.2, 27.2, 23.2, 22.4, 16.2.

Chiral HPLC (CHIRALCEL IA, 99\% hexane/1\% IPA, $1.0 \mathrm{~mL} / \mathrm{min}, 254 \mathrm{~nm}$, tR1 = 5.3 $\left.\min , t_{2}=6.0 \mathrm{~min}\right)$ indicated 99:01 er. $[\alpha] \mathbf{D 2 0}=+276.18\left(\mathrm{c}=0.25, \mathrm{CH}_{2} \mathrm{Cl}_{2}\right),[\alpha] \mathbf{D 2 0}=$ $+290.35\left(\mathrm{c}=0.45, \mathrm{CHCl}_{3}\right)$, while the literuate reported $(R, R)-2 \mathbf{r}[\alpha]_{\mathrm{D} 25}=-295.6(\mathrm{c}=1$, $\left.\mathrm{CHCl}_{3}\right), 99 \%$ ee. 2

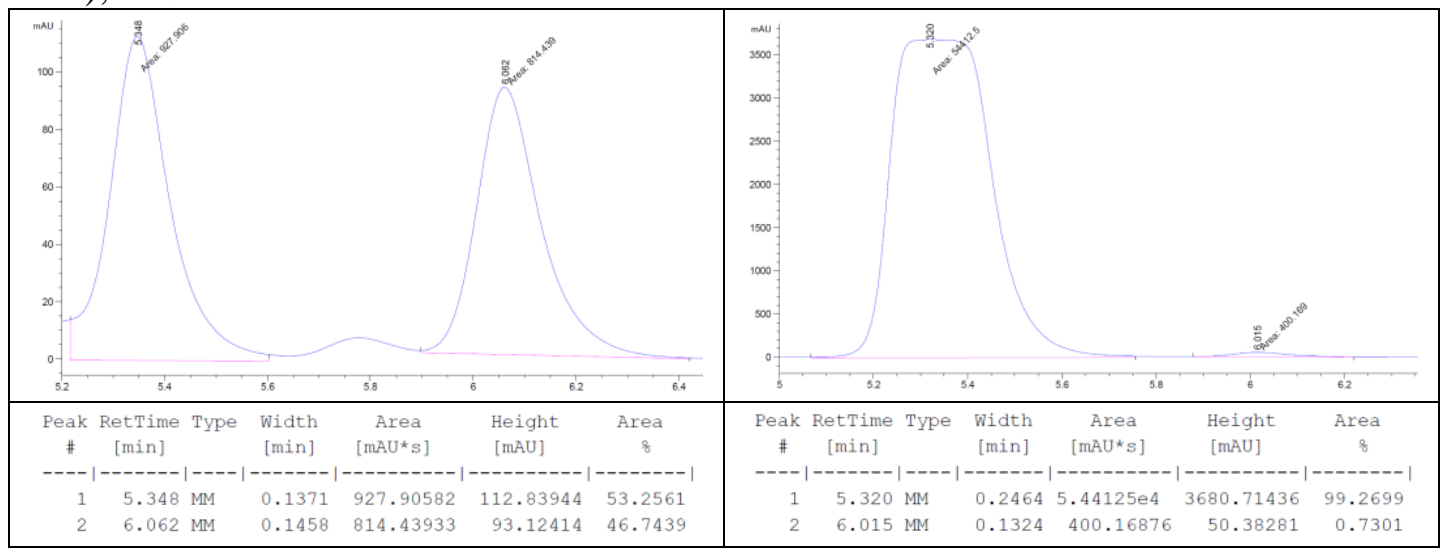

\section{Methyl 3-(((1R,2S)-2-methyl-2-phenylcyclopropyl)ethynyl)benzoate (2s)}

Alkynylated cyclopropane $2 \mathrm{~s}$ was prepared according to the general procedure. After standard work-up and purification, $2 \mathbf{s}$ was obtained as a colorless oil (42.3 $\mathrm{mg}, 73 \%$ yield).

1H NMR (600 MHz, CDCl3) $\delta 8.12(\mathrm{~s}, 1 \mathrm{H}), 7.97$ (d, $J=7.9 \mathrm{~Hz}, 1 \mathrm{H}), 7.63(\mathrm{~d}, J=7.7 \mathrm{~Hz}$, $1 \mathrm{H}), 7.40(\mathrm{t}, J=7.8 \mathrm{~Hz}, 1 \mathrm{H}), 7.37-7.31(\mathrm{~m}, 4 \mathrm{H}), 7.24(\mathrm{ddd}, J=8.5,5.4,3.3 \mathrm{~Hz}, 1 \mathrm{H})$, 
$3.95(\mathrm{~s}, 3 \mathrm{H}), 1.86(\mathrm{dd}, J=8.9,5.7 \mathrm{~Hz}, 1 \mathrm{H}), 1.66(\mathrm{~s}, 3 \mathrm{H}), 1.53(\mathrm{dd}, J=9.4,4.9 \mathrm{~Hz}, 1 \mathrm{H})$, $1.12-1.06(\mathrm{~m}, 1 \mathrm{H})$.

13C NMR (150 MHz, CDCl3) $\delta$ 166.5, 145.5, 135.8, 132.7, 130.3, 128.5, 128.4, 128.3, 127.3, 126.3, 124.4, 91.6, 78.4, 52.2, 28.1, 23.3, 22.6, 15.9. HRMS (TOF-MS ES+): $[\mathrm{M}+\mathrm{H}]_{+}$, calculated for $\mathrm{C}_{20} \mathrm{H}_{19} \mathrm{O}_{2}: 291.1380$; found 291.1375.

Chiral HPLC (CHIRALCEL IA, 99.8\% Hexane $/ 0.2 \%$ IA, $1.0 \mathrm{~mL} / \mathrm{min}, 254 \mathrm{~nm}$, tR1 = $23.6 \mathrm{~min}, \mathrm{tR2}=30.6 \mathrm{~min})$ indicated 96:04 er. $[\alpha]_{\mathbf{D} 20}=+350.23\left(\mathrm{c}=0.15, \mathrm{CH}_{2} \mathrm{Cl}_{2}\right)$.

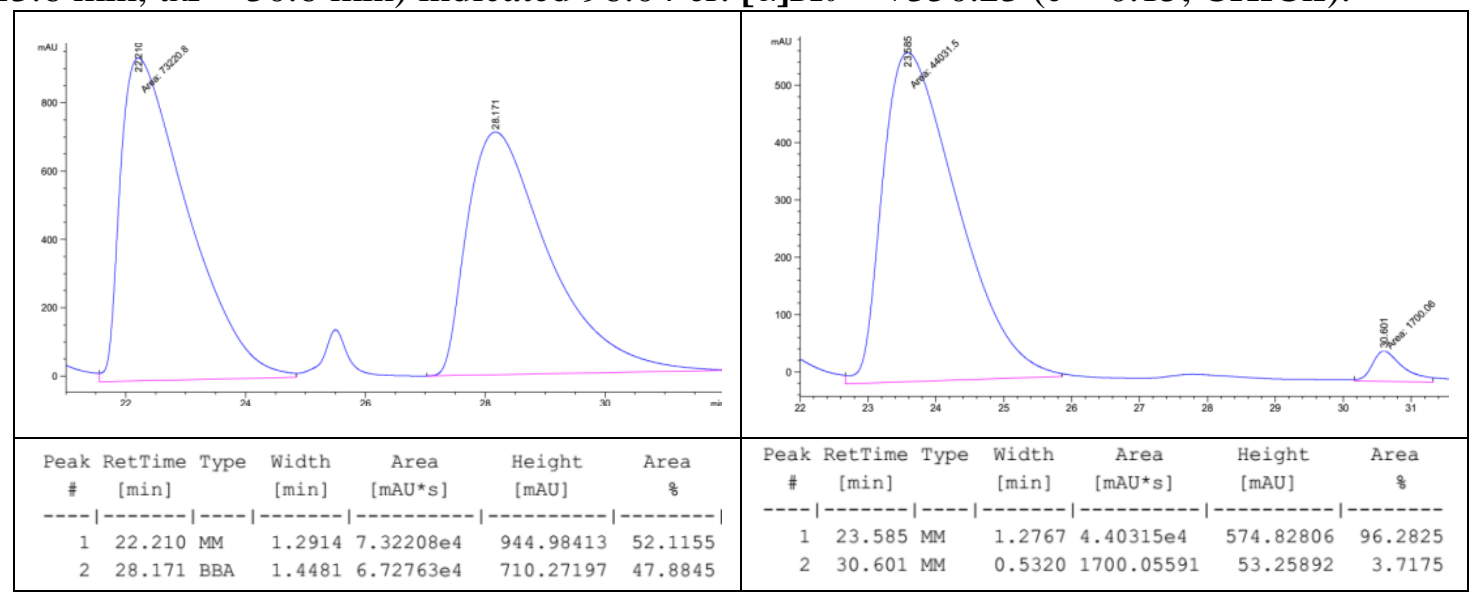

\section{2-(((1R,2S)-2-Methyl-2-phenylcyclopropyl)ethynyl)pyridine (2t)}

Alkynylated cyclopropane $2 \mathbf{t}$ was prepared according to the general procedure. After standard work-up and purification, $2 \mathbf{t}$ was obtained as a colorless oil $(35.0 \mathrm{mg}, 75 \%$ yield).

1H NMR (600 MHz, CDCl3) $\delta 8.12(\mathrm{~s}, 1 \mathrm{H}), 7.97(\mathrm{~d}, J=7.9 \mathrm{~Hz}, 1 \mathrm{H}), 7.63(\mathrm{~d}, J=7.7 \mathrm{~Hz}$, $1 \mathrm{H}), 7.40(\mathrm{t}, J=7.8 \mathrm{~Hz}, 1 \mathrm{H}), 7.37-7.31(\mathrm{~m}, 4 \mathrm{H}), 7.24(\mathrm{ddd}, J=8.5,5.4,3.3 \mathrm{~Hz}, 1 \mathrm{H})$, $3.95(\mathrm{~s}, 3 \mathrm{H}), 1.86(\mathrm{dd}, J=8.9,5.7 \mathrm{~Hz}, 1 \mathrm{H}), 1.66(\mathrm{~s}, 3 \mathrm{H}), 1.53(\mathrm{dd}, J=9.4,4.9 \mathrm{~Hz}, 1 \mathrm{H})$, 1.12 - 1.06 (m, 1H). 13C NMR (100 MHz, CDCl3) $\delta$ 149.6, 145.3, 143.7, 136.2, 128.4, 127.3, 126.9, 126.4, 122.2, 91.6, 78.9, 28.6, 23.2, 22.8, 15.6. HRMS (TOF-MS ES+):

$[\mathbf{M}+\mathbf{H}]_{+}$, calculated for $\mathrm{C}_{17} \mathrm{H}_{16} \mathrm{~N}$ : 234.1277 ; found 234.1269 .

Chiral HPLC (CHIRALCEL IA, 99\% Hexane/1\% IPA, $1.0 \mathrm{~mL} / \mathrm{min}, 254 \mathrm{~nm}, \mathrm{tR} 1=10.8$ min, tR2 $=19.4 \mathrm{~min})$ indicated 96:04 er. $[\alpha]_{\mathbf{D} 20}=+293.62\left(\mathrm{c}=0.35, \mathrm{CH}_{2} \mathrm{Cl}_{2}\right)$.

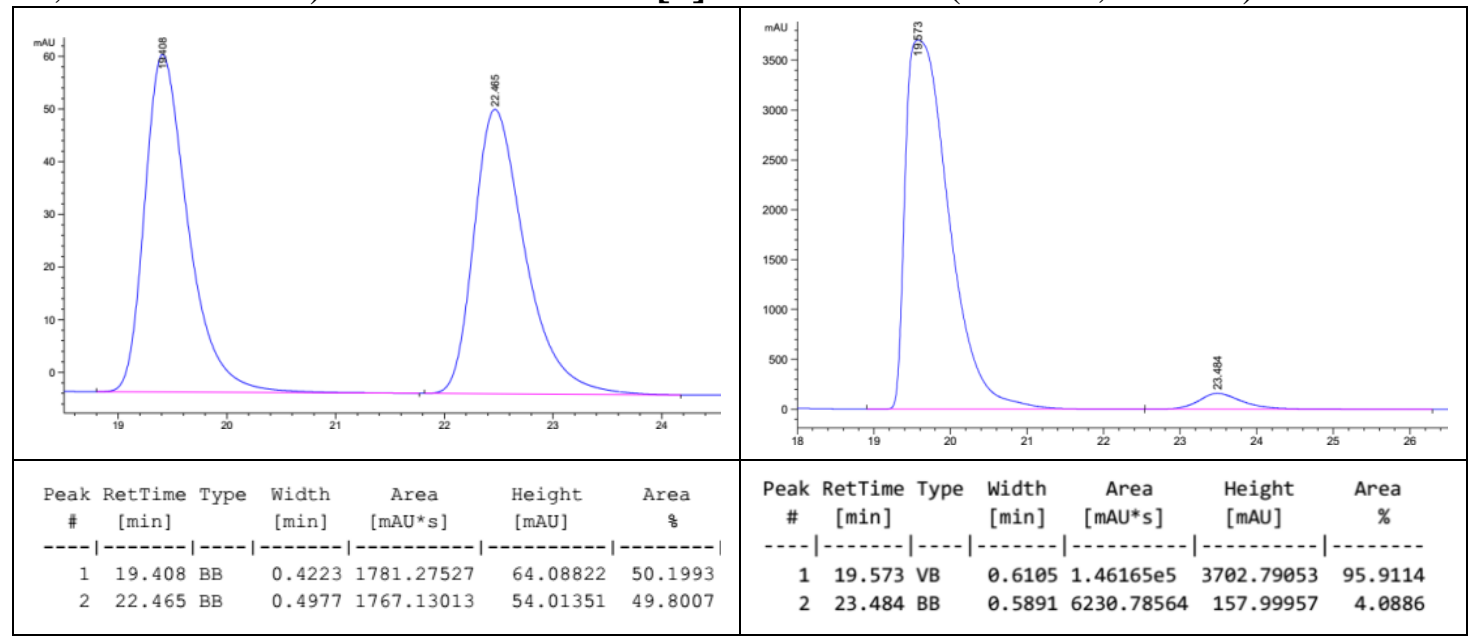


$2 \mathbf{u}$

Alkynylated cyclopropane $\mathbf{2} \mathbf{u}$ was prepared according to the general procedure. After standard work-up and purification, $\mathbf{2 u}$ was obtained as a colorless oil $(46.0 \mathrm{mg}, 68 \%$ yield).

$1 \mathrm{H}$ NMR $\left(600 \mathrm{MHz}, \mathbf{C D C l}_{3}\right) \delta 7.33(\mathrm{~d}, J=4.6 \mathrm{~Hz}, 4 \mathrm{H}), 7.23(\mathrm{td}, J=5.2,2.4 \mathrm{~Hz}, 1 \mathrm{H})$, $4.42(\mathrm{~d}, J=1.9 \mathrm{~Hz}, 2 \mathrm{H}), 4.23(\mathrm{~s}, 4 \mathrm{H}), 4.18(\mathrm{t}, J=1.7 \mathrm{~Hz}, 2 \mathrm{H}), 1.76(\mathrm{dd}, J=8.9,5.7 \mathrm{~Hz}$, 1H), $1.64(\mathrm{~s}, 3 \mathrm{H}), 1.47$ (dd, $J=8.9,4.5 \mathrm{~Hz}, 1 \mathrm{H}), 1.03-0.96(\mathrm{~m}, 1 \mathrm{H}) .13 \mathrm{C}$ NMR (150 MHz, CDCl3) $\delta$ 145.8, 128.4, 127.2, 126.2, 86.2, 71.4, 71.3, 69.8, 68.2, 68.2, 66.4, 27.3, 23.3, 22.5, 16.4 .

HRMS (TOF-MS ES+): [M+H]+, calculated for $\mathrm{C}_{22} \mathrm{H}_{21} \mathrm{Fe}$ : 341.0987 ; found 341.0979.

Chiral HPLC (CHIRALCEL IA, 99.8\% Hexane $/ 0.2 \%$ IPA, $1.0 \mathrm{~mL} / \mathrm{min}, 254 \mathrm{~nm}$, $\mathrm{tR}_{1}=$ $15.1 \mathrm{~min}, \mathrm{tR2}=19.8 \mathrm{~min})$ indicated 97:03 er. $[\alpha] \mathbf{D 2 0}=+199.75\left(\mathrm{c}=0.20, \mathrm{CH}_{2} \mathrm{Cl}_{2}\right)$.

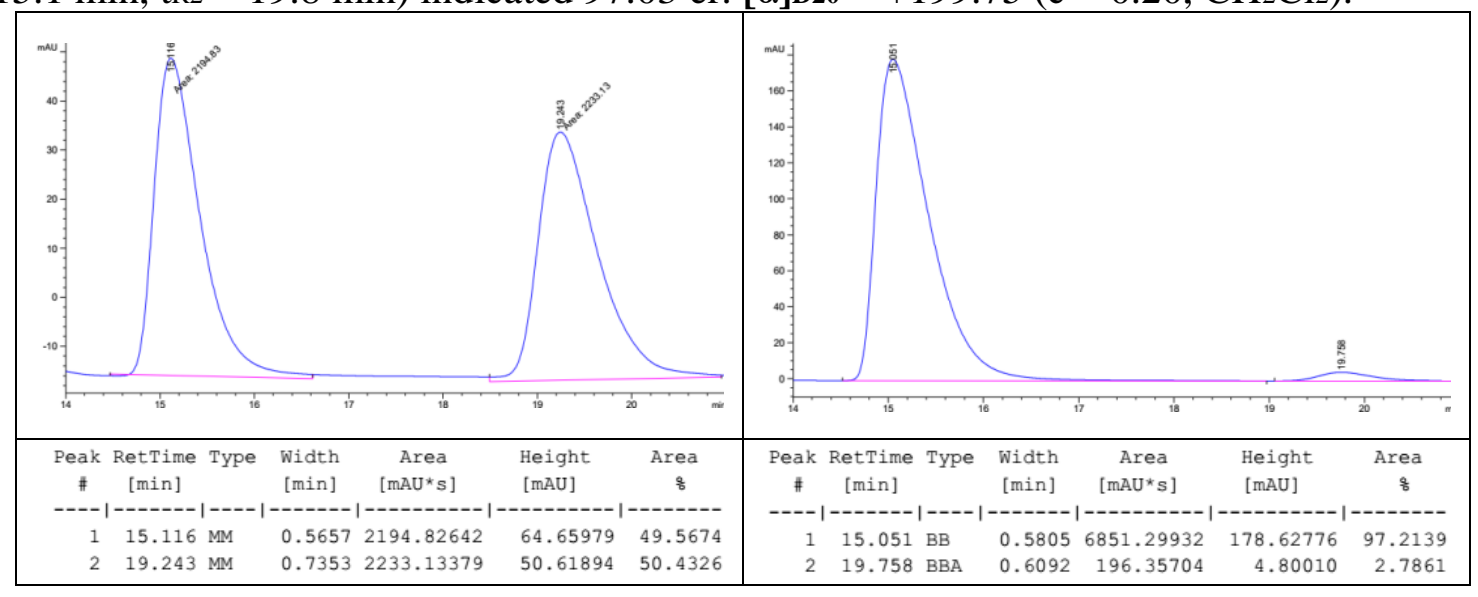

\section{1-(Dimethoxymethyl)-4-(((1R,2S)-2-methyl-2- phenylcyclopropyl)ethynyl)benzene (2v)}

Alkynylated cyclopropane $\mathbf{2 v}$ was prepared according to the general procedure. After standard work-up and purification, $\mathbf{2 v}$ was obtained as a colorless oil $(42.2 \mathrm{mg}, 69 \%$ yield).

1H NMR (600 MHz, CDCl3) $\delta 7.47(\mathrm{~d}, J=8.3 \mathrm{~Hz}, 2 \mathrm{H}), 7.41(\mathrm{~d}, J=8.1 \mathrm{~Hz}, 2 \mathrm{H}), 7.37$ $7.31(\mathrm{~m}, 4 \mathrm{H}), 7.24(\mathrm{td}, J=5.8,2.8 \mathrm{~Hz}, 1 \mathrm{H}), 5.42(\mathrm{~s}, 1 \mathrm{H}), 3.35(\mathrm{~s}, 6 \mathrm{H}), 1.86(\mathrm{dd}, J=8.9$, $5.7 \mathrm{~Hz}, 1 \mathrm{H}), 1.66(\mathrm{~s}, 3 \mathrm{H}), 1.52(\mathrm{dd}, J=9.1,4.7 \mathrm{~Hz}, 1 \mathrm{H}), 1.08(\mathrm{dd}, J=5.4,4.7 \mathrm{~Hz}, 1 \mathrm{H})$. 13C NMR (150 MHz, CDCl3) $\delta$ 145.6, 137.3, 131.4, 128.4, 127.3, 126.6, 126.3, 124.1, 102.6, 90.8, 79.1, 52.6, 28.0, 23.3, 22.6, 16.0.

HRMS (TOF-MS ES+): [M+H]+, calculated for $\mathrm{C}_{17} \mathrm{H}_{16} \mathrm{~N}$ : 307.1693; found 307.1688.

Chiral HPLC (CHIRALCEL IA, 99.8\% Hexane/0.2\% IPA, $1.0 \mathrm{~mL} / \mathrm{min}, 254 \mathrm{~nm}$, tR1 = $16.5 \mathrm{~min}, \mathrm{tR} 2=19.1 \mathrm{~min})$ indicated $96: 04 \mathrm{er} .[\alpha]_{\mathbf{D} 20}=+253.10\left(\mathrm{c}=0.32, \mathrm{CH}_{2} \mathrm{Cl}_{2}\right)$. 


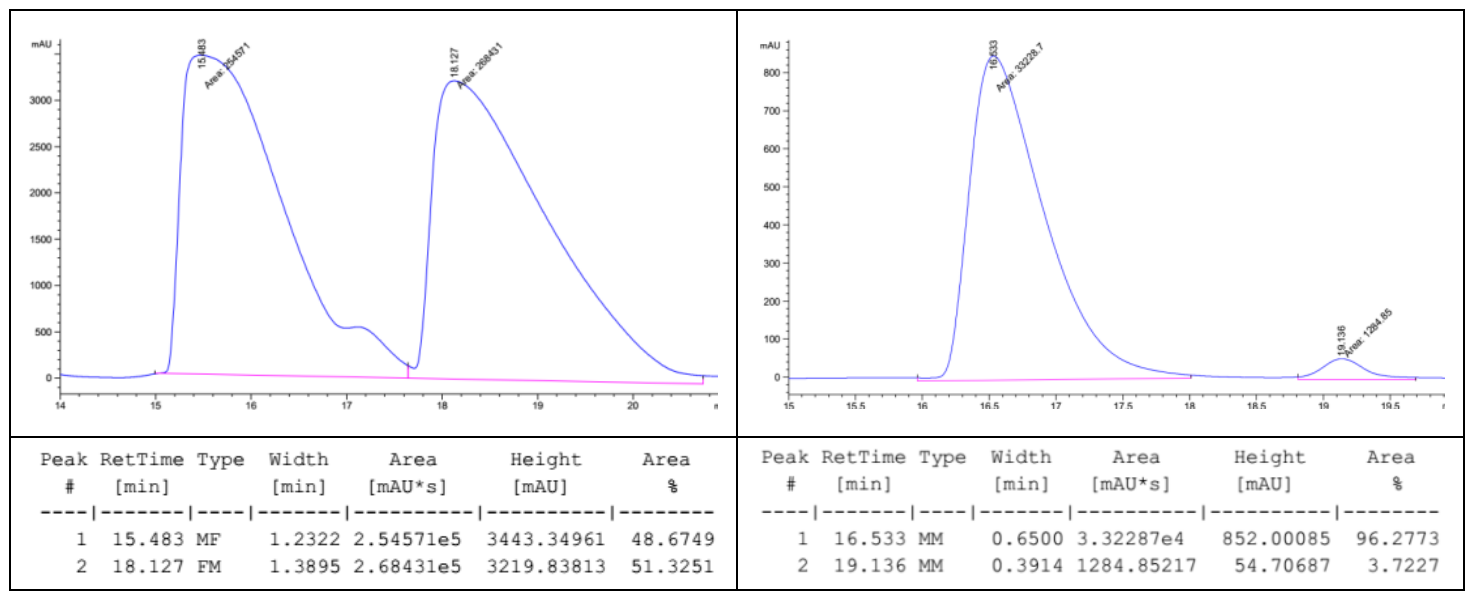

((1S,2S)-1-Methyl-2-(phenylbuta-1,3-diyn-1-yl)cyclopropyl)benzene (2w)

Alkynylated cyclopropane $\mathbf{2 w}$ was prepared according to the general procedure. After standard work-up and purification, $\mathbf{2} \mathbf{w}$ was obtained as a colorless oil $(33.3 \mathrm{mg}, 65 \%$ yield).

1H NMR (400 MHz, CDCl3) $\delta$ 7.32-7.28 (m, 2H), 7.15-7.00 (m, 8H), $1.59(\mathrm{dd}, J=5.6$, $8.9 \mathrm{~Hz}, 1 \mathrm{H}), 1.42(\mathrm{~s}, 3 \mathrm{H}), 1.31(\mathrm{dd}, J=4.6,8.9 \mathrm{~Hz}, 1 \mathrm{H}), 0.89(\mathrm{dd}, J=4.6,5.6 \mathrm{~Hz}, 1 \mathrm{H})$. 13C NMR (100 MHz, CDCl3) $\delta 144.9,132.5,128.8,128.5,128.3,127.2,126.5,122.1$, 84.9, 63.7, 29.2, 23.5, 22.7, 16.0. HRMS (TOF-MS ES+): [M+H]+, calculated for $\mathrm{C}_{20} \mathrm{H}_{17}$ : 257.1325; found 257.1338.

Chiral HPLC (CHIRALCEL IC, 100\% hexane, $1.0 \mathrm{~mL} / \mathrm{min}, 254 \mathrm{~nm}$, tR1 = $27.0 \mathrm{~min}$, tR2 $=29.4 \mathrm{~min})$ indicated 97:03 er. $[\alpha]]_{\mathbf{D 2 0}}=+285.38\left(\mathrm{c}=0.39, \mathrm{CH}_{2} \mathrm{Cl}_{2}\right)$.

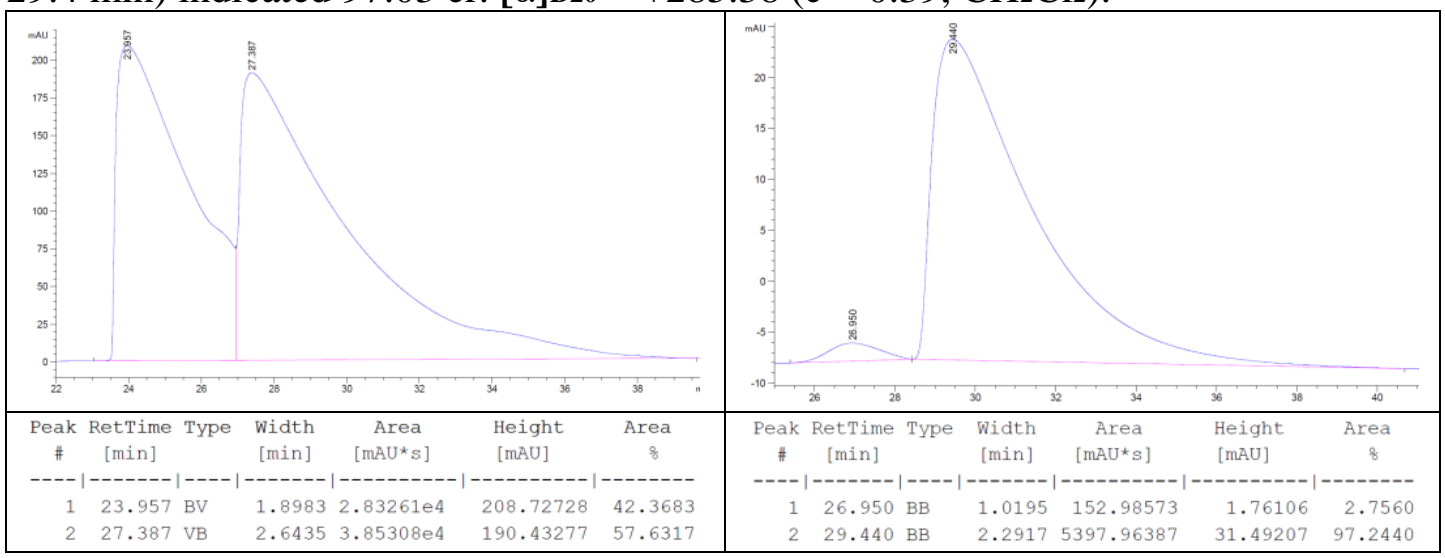

\section{1,2-Dimethoxy-4-(((1S,2S)-2-methyl-2-phenylcyclopropyl)buta-1,3-diyn- 1-yl)benzene (2x)}

Alkynylated cyclopropane $\mathbf{2} \mathbf{x}$ was prepared according to the general procedure. After standard work-up and purification, $\mathbf{2 x}$ was obtained as a colorless oil $(36.0 \mathrm{mg}, 57 \%$ yield).

1H NMR (400 MHz, CDCl3) $\delta$ 7.24-7.10 (m, 5H), 7.03 (d, J = 8.3 Hz, 1H), $6.90(\mathrm{~s}, 1 \mathrm{H})$, $6.71(\mathrm{~d}, J=8.3 \mathrm{~Hz}, 1 \mathrm{H}), 3.80(\mathrm{~s}, 3 \mathrm{H}), 3.78(\mathrm{~s}, 3 \mathrm{H}), 1.69(\mathrm{dd}, J=5.7,8.9 \mathrm{~Hz}, 1 \mathrm{H}), 1.52(\mathrm{~s}$, $3 \mathrm{H}), 1.41(\mathrm{dd}, J=4.6,8.9 \mathrm{~Hz}, 1 \mathrm{H}), 0.99(\mathrm{dd}, J=4.8,4.8 \mathrm{~Hz}, 1 \mathrm{H}) .13 \mathrm{C}$ NMR (100 MHz, 
CDCl3) $\delta 150.0,148.5,144.9,128.4,127.2,126.5,126.1,114.9,114.1,111.0,84.3,74.9$, 73.3, 63.8, 55.8, 29.0, 23.5, 22.6, 16.0. HRMS (TOF-MS ES+): [M+H]+, calculated for $\mathrm{C}_{22} \mathrm{H}_{21} \mathrm{O}_{2}: 317.1536$; found 317.1548.

Chiral HPLC (CHIRALCEL ADH, 99\% Hexane $/ 1 \%$ IPA, $1.0 \mathrm{~mL} / \mathrm{min}, 254 \mathrm{~nm}$, tR1 = $10.8 \mathrm{~min}, \mathrm{tR2}=19.4 \mathrm{~min})$ indicated 97:03 er. $[\alpha]_{\mathbf{D} 20}=+293.62\left(\mathrm{c}=0.35, \mathrm{CH}_{2} \mathrm{Cl}_{2}\right)$.

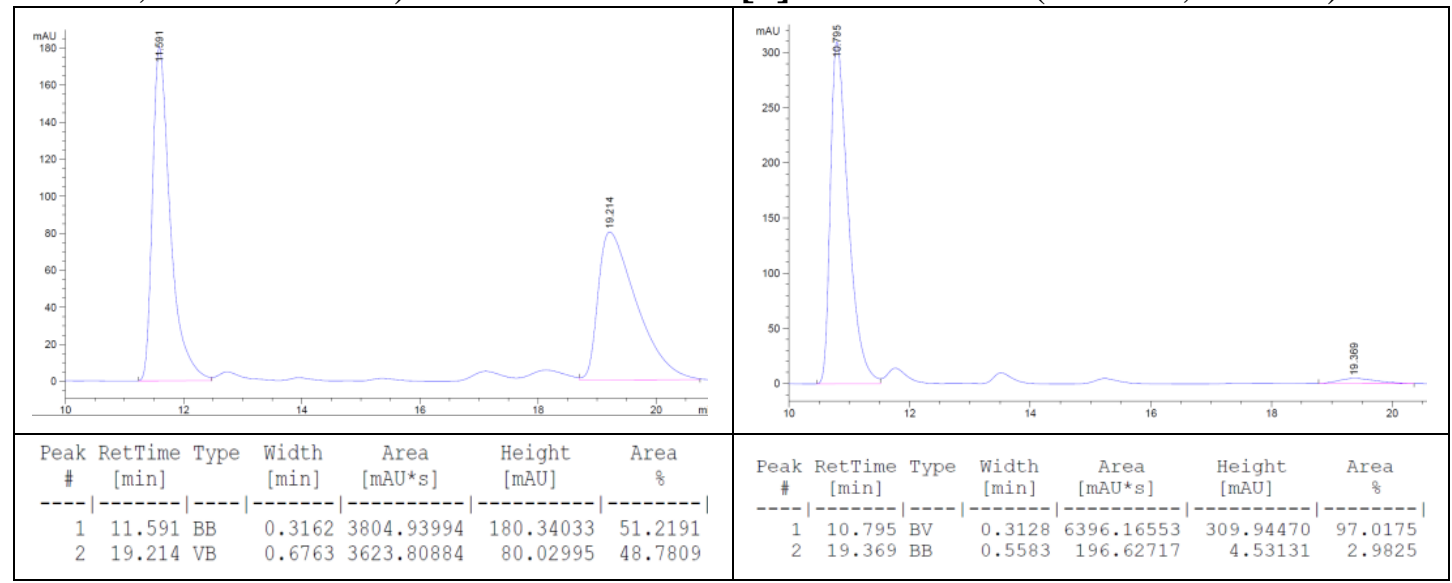

\section{$((1 R, 2 R)-1-M e t h y l-2-((E)-4-p h e n y l b u t-3-e n-1-y n-1-$ yl)cyclopropyl)benzene (3a)}

Alkynylated cyclopropane 3a was prepared according to the general procedure. After standard work-up and purification, 3a was obtained as a colorless oil $(29.5 \mathrm{mg}, 57 \%$ yield).

$1 \mathbf{H}$ NMR $\left(600 \mathrm{MHz}, \mathrm{CDCl}_{3}\right) \delta 7.41(\mathrm{~d}, J=7.3 \mathrm{~Hz}, 2 \mathrm{H}), 7.37-7.32(\mathrm{~m}, 6 \mathrm{H}), 7.31-7.29$ $(\mathrm{m}, 1 \mathrm{H}), 7.26-7.21(\mathrm{~m}, 1 \mathrm{H}), 6.94(\mathrm{~d}, J=16.2 \mathrm{~Hz}, 1 \mathrm{H}), 6.24(\mathrm{dd}, J=16.2,2.0 \mathrm{~Hz}, 1 \mathrm{H})$, 1.82 (ddd, $J=8.9,5.6,2.0 \mathrm{~Hz}, 1 \mathrm{H}), 1.62(\mathrm{~s}, 3 \mathrm{H}), 1.51(\mathrm{dd}, J=9.0,4.4 \mathrm{~Hz}, 1 \mathrm{H}), 1.04(\mathrm{dd}$, $J=5.7,4.5 \mathrm{~Hz}, 1 \mathrm{H}) .13 \mathrm{C} \mathbf{N M R}\left(150 \mathrm{MHz}, \mathrm{CDCl}_{3}\right) \delta 145.6,140.1,136.5,128.6,128.4$, 128.2, 127.3, 126.3, 126.0, 108.7, 93.0, 78.7, 28.2, 23.4, 22.6, 16.3. HRMS (TOF-MS

$\left.\mathbf{E S}_{+}\right):[\mathbf{M + H}]_{+}$, calculated for $\mathrm{C}_{20} \mathrm{H}_{19}$ : 259.1481; found 259.1466 .

Chiral HPLC (CHIRALCEL IA, 100\% hexane, $1.0 \mathrm{~mL} / \mathrm{min}, 254 \mathrm{~nm}$, tR $1=18.3 \mathrm{~min}$, tR2 $=25.3 \mathrm{~min})$ indicated $94: 06 \mathrm{er} .[\alpha] \mathbf{D 2 0}=+282.15\left(\mathrm{c}=0.40, \mathrm{CH}_{2} \mathrm{Cl}_{2}\right)$.

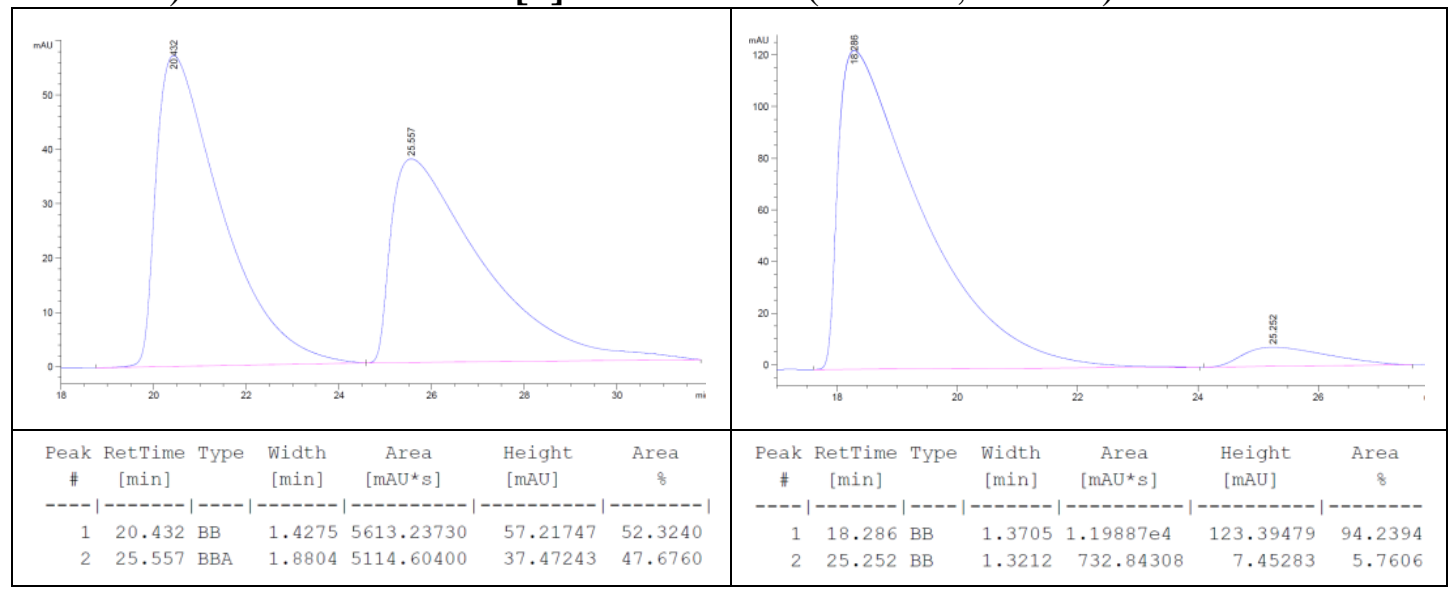




\section{1,2-Dimethoxy-4-((E)-4-((1R,2R)-2-methyl-2-phenylcyclopropyl)but-1- en-3-yn-1-yl)benzene (3b)}

Alkynylated cyclopropane $\mathbf{3 b}$ was prepared according to the general procedure. After standard work-up and purification, $3 \mathbf{b}$ was obtained as a colorless oil $(29.3 \mathrm{mg}, 46 \%$ yield).

1H NMR (600 MHz, CDCl 3$) \delta 7.35-7.30$ (m, 4H), $7.23(\mathrm{ddd}, J=5.5,4.1,2.5 \mathrm{~Hz}, 1 \mathrm{H})$, $6.95(\mathrm{~d}, J=8.2 \mathrm{~Hz}, 2 \mathrm{H}), 6.91-6.82(\mathrm{~m}, 2 \mathrm{H}), 6.10(\mathrm{dd}, J=16.2,2.0 \mathrm{~Hz}, 1 \mathrm{H}), 3.92(\mathrm{~s}$, $3 \mathrm{H}), 3.91(\mathrm{~s}, 3 \mathrm{H}), 1.82(\mathrm{ddd}, J=9.0,5.7,2.0 \mathrm{~Hz}, 1 \mathrm{H}), 1.62(\mathrm{~s}, 3 \mathrm{H}), 1.50(\mathrm{dd}, J=8.9,4.5$ $\mathrm{Hz}, 1 \mathrm{H}), 1.02$ (dd, $J=5.7,4.5 \mathrm{~Hz}, 1 \mathrm{H}) .13 \mathrm{C} \mathrm{NMR}\left(150 \mathrm{MHz}, \mathrm{CDCl}_{3}\right) \delta 149.4,149.0$, $145.6,139.9,129.7,128.4,127.2,126.2,119.5,111.0,108.1,106.7,92.3,78.7,55.9$, 55.8, 28.1, 23.4, 22.6, 16.3.

HRMS (TOF-MS ES+): [M+H]+, calculated for $\mathrm{C}_{22} \mathrm{H}_{23} \mathrm{O}_{2}: 319.1693$; found 319.1677 .

Chiral HPLC (CHIRALCEL IA, 98\% hexane/2\% IPA, $1.0 \mathrm{~mL} / \mathrm{min}, 254 \mathrm{~nm}$, tR1 = 13.9 $\left.\min , \mathrm{tR}_{2}=19.5 \mathrm{~min}\right)$ indicated 94:06 er. $[\alpha]_{\mathbf{D} 20}=+275.63\left(\mathrm{c}=0.28, \mathrm{CH}_{2} \mathrm{Cl}_{2}\right)$.

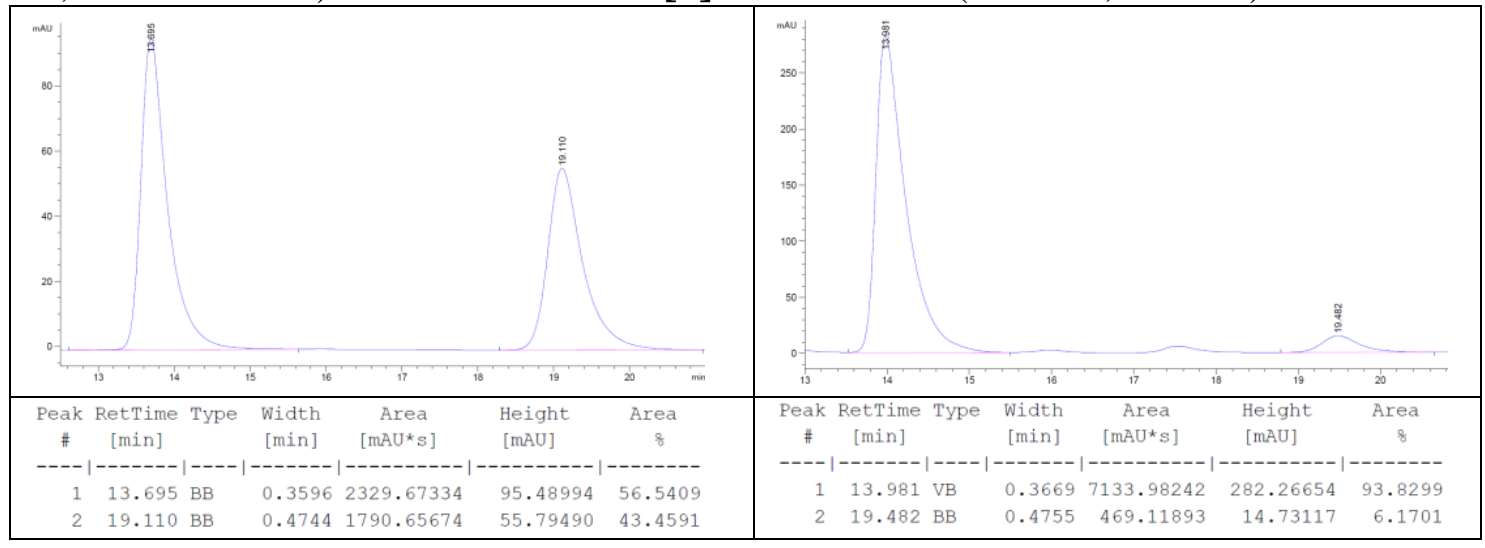

\section{1-Methoxy-4-((E)-4-((1R,2R)-2-methyl-2-phenylcyclopropyl)but-1-en-3- yn-1-yl)benzene (3c)}

Alkynylated cyclopropane 3c was prepared according to the general procedure. After standard work-up and purification, 3c was obtained as a colorless oil (24.8 mg, 43\% yield).

1 H NMR $\left(400 \mathrm{MHz}, \mathrm{CDCl}_{3}\right) \delta 7.26-7.18(\mathrm{~m}, 6 \mathrm{H}), 7.12(\mathrm{dd}, J=8.9,4.6 \mathrm{~Hz}, 1 \mathrm{H}), 6.82-$ $6.74(\mathrm{~m}, 3 \mathrm{H}), 5.99$ (dd, $J=16.2,2.0 \mathrm{~Hz}, 1 \mathrm{H}), 3.73(\mathrm{~s}, 3 \mathrm{H}), 1.74-1.69(\mathrm{~m}, 1 \mathrm{H}), 1.51$ (s, $3 \mathrm{H}), 1.38(\mathrm{dd}, J=8.9,4.5 \mathrm{~Hz}, 1 \mathrm{H}), 0.92(\mathrm{dd}, J=5.6,4.6 \mathrm{~Hz}, 1 \mathrm{H}) .13 \mathrm{C}$ NMR $(100 \mathrm{MHz}$, $\left.\mathrm{CDCl}_{3}\right) \delta 159.8,145.7,139.7,129.4,128.4,127.3,127.2,126.2,114.1,106.4,92.1,78.9$, 55.3, 28.0, 23.3, 22.6, 16.3 .

HRMS (TOF-MS ES+): [M+H] $]_{+}$, calculated for $\mathrm{C}_{21} \mathrm{H}_{21} \mathrm{O}$ : 289.1587; found 289.1575.

Chiral HPLC (CHIRALCEL IC, 99.8\% Hexane/0.2\% IPA, $1.0 \mathrm{~mL} / \mathrm{min}, 254 \mathrm{~nm}$, tR1 = $14.1 \mathrm{~min}, \mathrm{tR} 2=16.5 \mathrm{~min})$ indicated 95:05 er. $[\alpha] \mathbf{D 2 0}=+265.31\left(\mathrm{c}=0.39, \mathrm{CH}_{2} \mathrm{Cl}_{2}\right)$. 


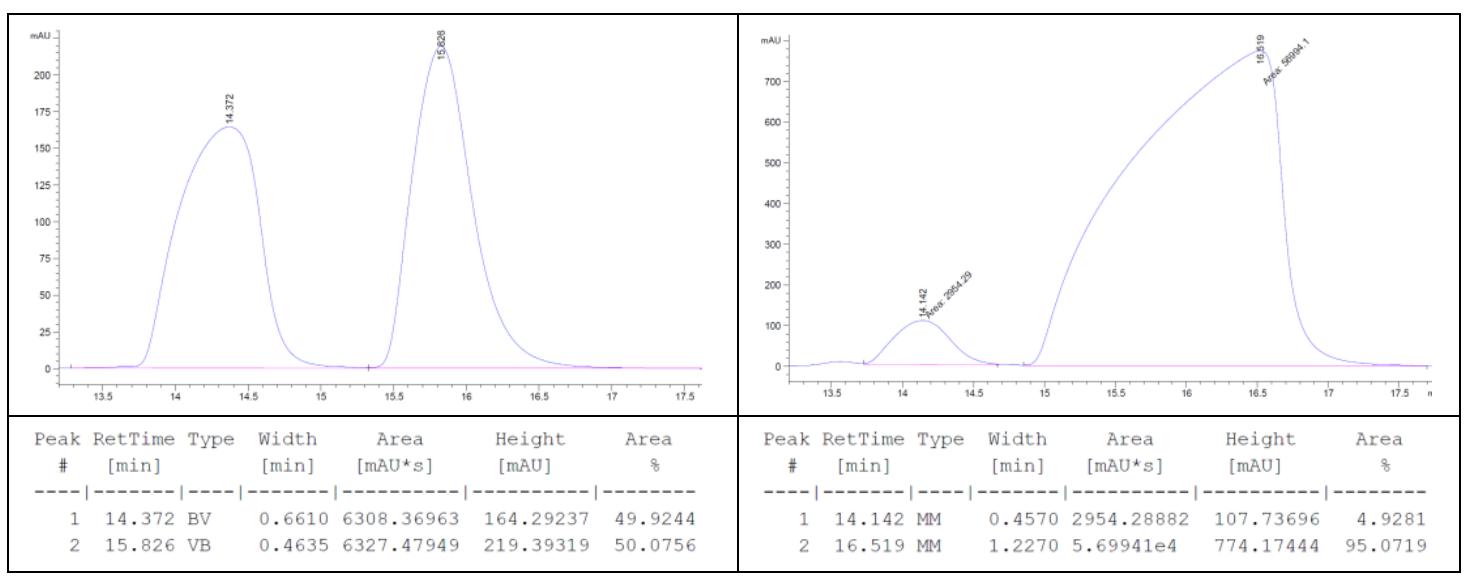

\section{1-Chloro-4-((E)-4-((1R,2R)-2-methyl-2-phenylcyclopropyl)but-1-en-3- yn-1-yl)benzene (3d)}

Alkynylated cyclopropane 3d was prepared according to the general procedure. After standard work-up and purification, 3d was obtained as a colorless oil $(27.5 \mathrm{mg}, 47 \%$ yield).

1H NMR (400 MHz, CDCl3) $\delta$ 7.25-7.10 (m, 8H), 7.15-7.12 (m, 1H), 6.77 (d, $J=16.2$ $\mathrm{Hz}, 1 \mathrm{H}), 6.10(\mathrm{dd}, J=2.0,16.2 \mathrm{~Hz}, 1 \mathrm{H}), 1.74-1.69(\mathrm{~m}, 1 \mathrm{H}), 1.51(\mathrm{~s}, 3 \mathrm{H}), 1.41$ (dd, $J=$ 4.5, 8.9 Hz, 1H), $0.93(\mathrm{dd}, J=4.6,5.6 \mathrm{~Hz}, 1 \mathrm{H})$. 13C NMR (100 MHz, CDCl3) $\delta 145.5$, 138.8, 135.1, 133.9, 128.9, 128.4, 127.2, 127.2, 126.3, 109.5, 93.7, 78.4, 28.2, 23.4, 22.6, 16.2. HRMS (TOF-MS ES+): [M+H]+, calculated for $\mathrm{C}_{20} \mathrm{H}_{18} \mathrm{Cl}$ : 293.1092; found 293.1070.

Chiral HPLC (CHIRALCEL IC, 99.8\% Hexane/0.2\% IPA, $1.0 \mathrm{~mL} / \mathrm{min}, 254 \mathrm{~nm}$, tR1 = $11.7 \mathrm{~min}, \mathrm{tR} 2=13.8 \mathrm{~min})$ indicated 98:02 er. $[\alpha]_{\mathbf{D} 20}=+243.68\left(\mathrm{c}=0.23, \mathrm{CH}_{2} \mathrm{Cl}_{2}\right)$.

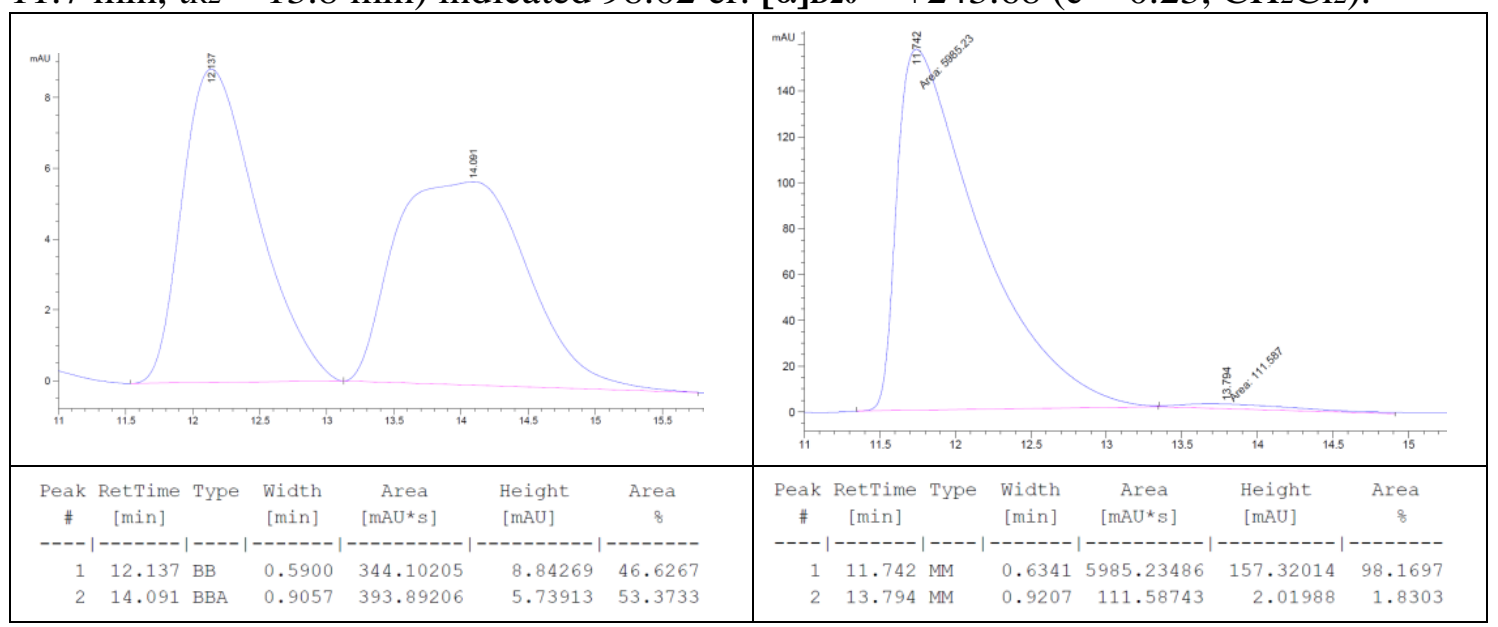

\section{1-Bromo-4-((E)-4-((1R,2R)-2-methyl-2-phenylcyclopropyl)but-1-en-3- yn-1-yl)benzene (3e)}

Alkynylated cyclopropane 3e was prepared according to the general procedure. After standard work-up and purification, $3 \mathbf{e}$ was obtained as a colorless oil $(33.7 \mathrm{mg}, 50 \%$ yield). 
1H NMR (400 MHz, CDCls) $\delta 7.35(\mathrm{~d}, J=8.4 \mathrm{~Hz}, 2 \mathrm{H}), 7.22-7.19(\mathrm{~m}, 4 \mathrm{H}), 7.16-7.10$ $(\mathrm{m}, 3 \mathrm{H}), 6.74(\mathrm{~d}, J=16.2 \mathrm{~Hz}, 1 \mathrm{H}), 6.11(\mathrm{dd}, J=2.0,16.2 \mathrm{~Hz}, 1 \mathrm{H}), 1.70(\mathrm{~m}, 1 \mathrm{H}), 1.51(\mathrm{~s}$, $3 \mathrm{H}), 1.40(\mathrm{dd}, J=4.5,8.9 \mathrm{~Hz}, 1 \mathrm{H}), 0.92(\mathrm{dd}, J=4.6,5.6 \mathrm{~Hz}, 1 \mathrm{H}) .13 \mathrm{C}$ NMR (100 MHz, CDCl3) $\delta 145.5,138.8,135.5,131.8,128.4,127.4,127.2,126.3,122.0,109.6,93.8,78.4$, 28.2, 23.4, 22.6, 16.3. HRMS (TOF-MS $\mathbf{E S} \mathbf{S}_{+}$: $[\mathbf{M}+\mathbf{H}]_{+}$, calculated for $\mathrm{C}_{20} \mathrm{H}_{18} \mathrm{Br}$ : 337.0586; found 337.0563.

Chiral HPLC (CHIRALCEL IC, 99.8\% Hexane/0.2\% IPA, $1.0 \mathrm{~mL} / \mathrm{min}, 254 \mathrm{~nm}$, tR1 = $17.1 \mathrm{~min}, \mathrm{tR} 2=18.7 \mathrm{~min})$ indicated 98:02 er. $[\alpha] \mathbf{D} 20=+337.99\left(\mathrm{c}=1.13, \mathrm{CH}_{2} \mathrm{Cl}_{2}\right)$.

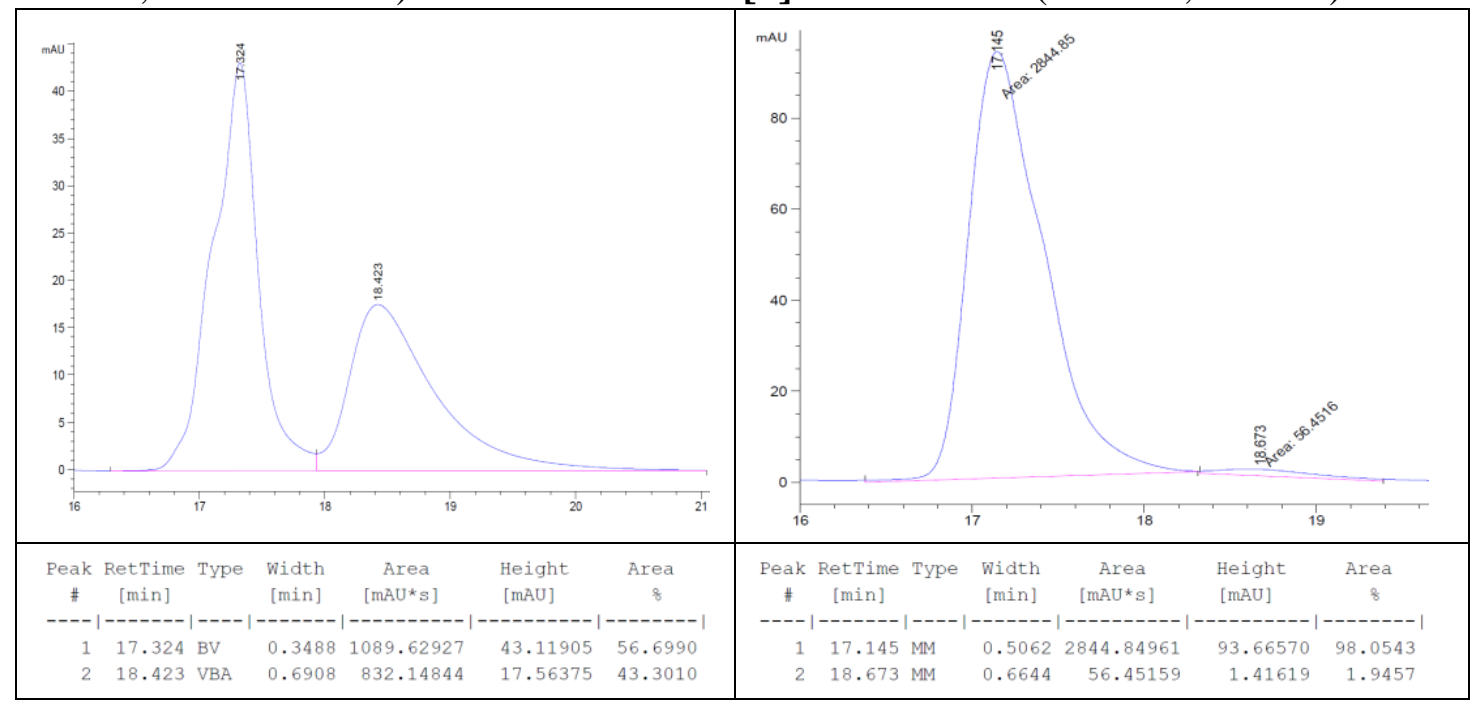

\section{1-((E)-4-((1R,2R)-2-Methyl-2-phenylcyclopropyl)but-1-en-3-yn-1- yl)naphthalene (3f)}

Alkynylated cyclopropane 3f was prepared according to the general procedure. After standard work-up and purification, 3f was obtained as a colorless oil $(29.0 \mathrm{mg}, 47 \%$ yield).

1H NMR (400 MHz, CDCl3) 7.73-7.60 (m, 4H), 7.48 (dd, $J=1.7,8.6 \mathrm{~Hz}, 1 \mathrm{H}), 7.40-$ $7.33(\mathrm{~m}, 2 \mathrm{H}), 7.22(\mathrm{~d}, J=4.0 \mathrm{~Hz}, 4 \mathrm{H}), 7.15-7.10(\mathrm{~m}, 1 \mathrm{H}), 6.98(\mathrm{~d}, J=16.2 \mathrm{~Hz}, 1 \mathrm{H}), 6.25$ (dd, $J=2.0,16.2 \mathrm{~Hz}, 1 \mathrm{H}), 1.76-1.72(\mathrm{~m}, 1 \mathrm{H}), 1.53(\mathrm{~s}, 3 \mathrm{H}), 1.41(\mathrm{dd}, J=4.6,9.0 \mathrm{~Hz}, 1 \mathrm{H})$, 0.95 (dd, $J=4.6,5.6 \mathrm{~Hz}, 1 \mathrm{H}) .13 \mathrm{C}$ NMR $(\mathbf{1 0 0} \mathbf{~ M H z}, \mathbf{C D C l} 3) \delta 145.6,140.2,134.1$, 133.5, 133.3, 128.4, 128.3, 127.7, 127.2, 126.4, 126.3, 126.1, 122.7, 109.1, 93.5, 78.9, 28.2, 23.4, 22.6, 16.4. HRMS (TOF-MS ES+): $[\mathbf{M + H}]_{+}$, calculated for $\mathrm{C}_{24} \mathrm{H}_{21}$ : 309.1638; found 309.1607.

Chiral HPLC (CHIRALCEL IC, 99.8\% Hexane/0.2\% IPA, $1.0 \mathrm{~mL} / \mathrm{min}, 254 \mathrm{~nm}$, tR1 $=$ $18.1 \mathrm{~min}, \mathrm{tR2}=20.0 \mathrm{~min})$ indicated 90:10 er. $[\alpha] \mathbf{D 2 0}=+294.46\left(\mathrm{c}=0.90, \mathrm{CH}_{2} \mathrm{Cl}_{2}\right)$. 


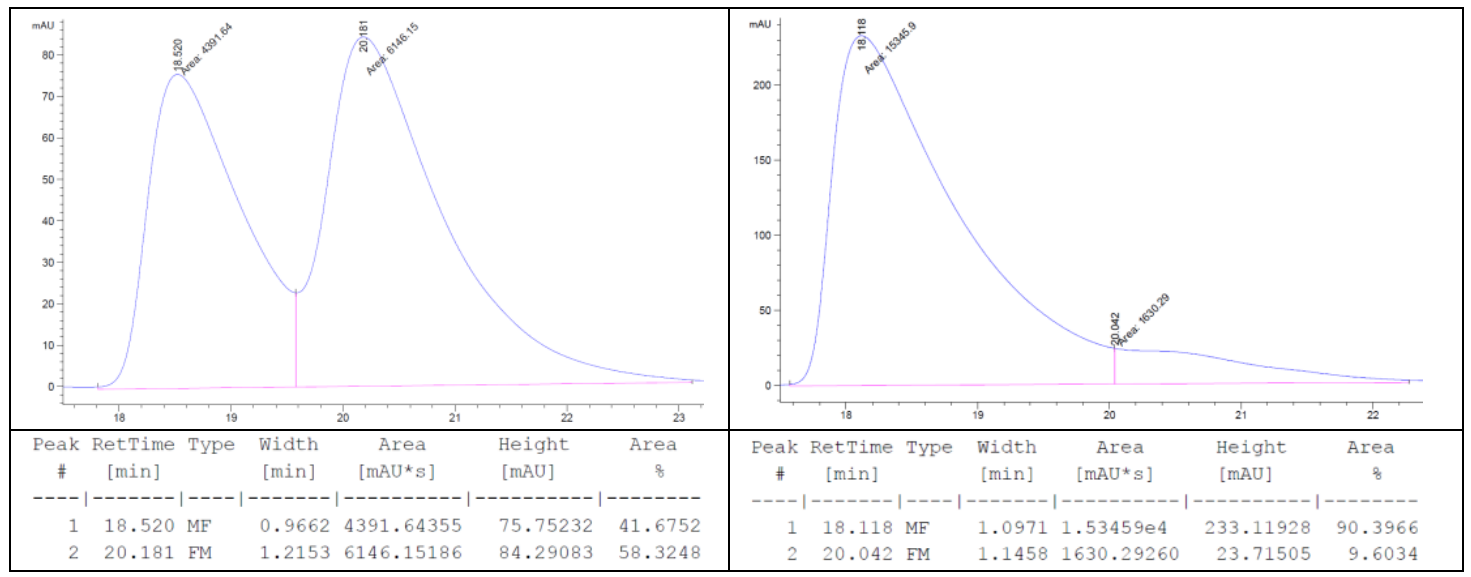

\section{4-((E)-4-((1R,2R)-2-Methyl-2-phenylcyclopropyl)but-1-en-3-yn-1-yl)- 1,1'-biphenyl (3g)}

Alkynylated cyclopropane $\mathbf{3 g}$ was prepared according to the general procedure. After standard work-up and purification, $\mathbf{3 g}$ was obtained as a colorless oil (36.8 mg, 55\% yield).

1H NMR (400 MHz, CDCl3) $\delta$ 7.55-7.45 (m, 4H), 7.40-7.30 (m, 4H), 7.28-7.20 (m, 5H), $7.16-7.13(\mathrm{~m}, 1 \mathrm{H}), 6.87(\mathrm{~d}, J=16.2 \mathrm{~Hz}, 1 \mathrm{H}), 6.17(\mathrm{dd}, J=2.0,16.2 \mathrm{~Hz}, 1 \mathrm{H}), 1.76-1.70$ $(\mathrm{m}, 1 \mathrm{H}), 1.53(\mathrm{~s}, 3 \mathrm{H}), 1.41(\mathrm{dd}, J=4.5,8.9 \mathrm{~Hz}, 1 \mathrm{H}), 0.95(\mathrm{dd}, J=4.6,5.6 \mathrm{~Hz}, 1 \mathrm{H}) .{ }_{13} \mathrm{C}$ NMR (100 MHz, CDCl3) $\delta 145.6,141.0,140.5,139.7,135.6,128.8,128.4,127.4,127.3$, $127.3,126.9,126.5,126.3,108.8,93.3,78.8,28.2,23.4,22.6,16.3$.

HRMS (TOF-MS ES+): [M+H]+, calculated for $\mathrm{C}_{26} \mathrm{H}_{23}$ : 335.1794; found 335.1781.

Chiral HPLC (CHIRALCEL IC, 99.8\% hexane/0.2\% IPA, $1.0 \mathrm{~mL} / \mathrm{min}, 254 \mathrm{~nm}$, tR1 = $20.2 \mathrm{~min}, \mathrm{tR} 2=30.9 \mathrm{~min})$ indicated 99:01 er. $[\alpha]_{\mathbf{D} 20}=+218.17\left(\mathrm{c}=0.33, \mathrm{CH}_{2} \mathrm{Cl}_{2}\right)$.

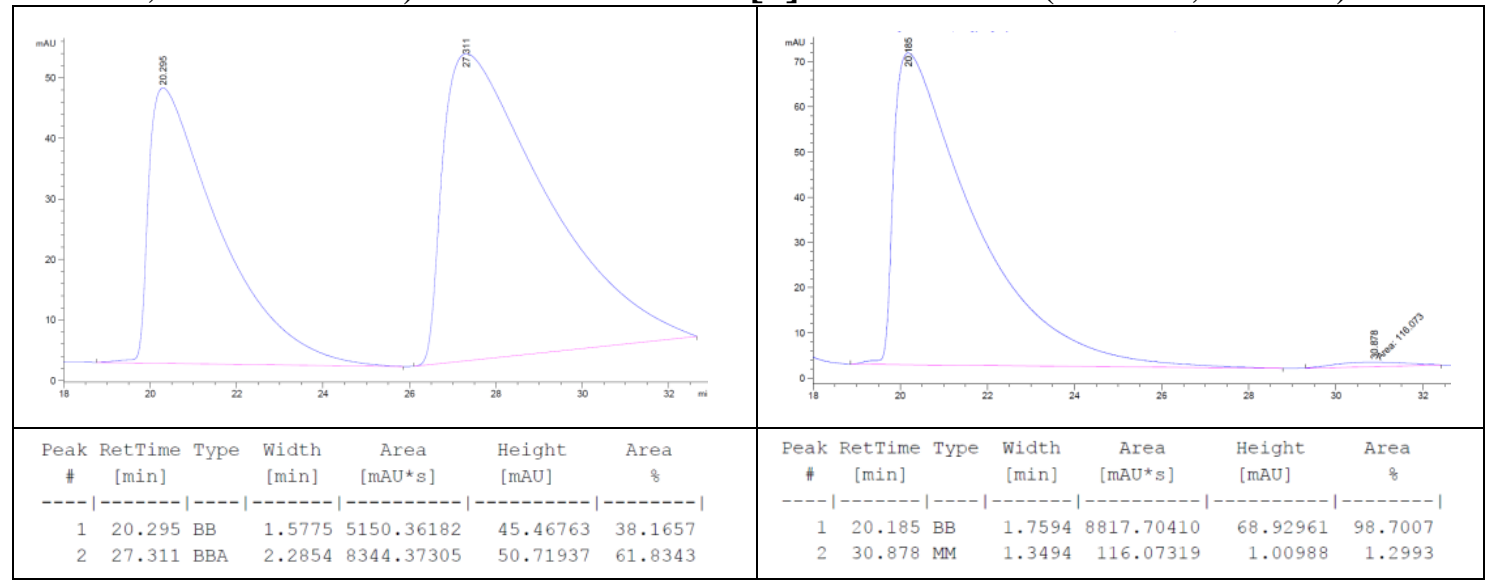

\section{1-Bromo-4-((E)-4-((1R,2R)-2-methyl-2-(p-tolyl)cyclopropyl)but-1-en-3- yn-1-yl)benzene (3h)}

Alkynylated cyclopropane $\mathbf{3 h}$ was prepared according to the general procedure. After standard work-up and purification, 3h was obtained as a colorless oil (40.6 mg, 58\% yield). 
1H NMR (400 MHz, CDCl3) $\delta 7.36(\mathrm{~d}, J=8.4 \mathrm{~Hz}, 2 \mathrm{H}), 7.16-7.10(\mathrm{~m}, 4 \mathrm{H}), 7.03(\mathrm{~d}, J=$ $8.0 \mathrm{~Hz}, 2 \mathrm{H}), 6.74(\mathrm{~d}, J=16.2 \mathrm{~Hz}, 1 \mathrm{H}), 6.12(\mathrm{dd}, J=2.0,16.2 \mathrm{~Hz}, 1 \mathrm{H}), 2.25(\mathrm{~s}, 3 \mathrm{H}), 1.70-$ $1.65(\mathrm{~m}, 1 \mathrm{H}), 1.50(\mathrm{~s}, 3 \mathrm{H}), 1.38(\mathrm{dd}, J=4.2,8.7 \mathrm{~Hz}, 1 \mathrm{H}), 0.90(\mathrm{dd}, J=4.6,5.5 \mathrm{~Hz}, 1 \mathrm{H})$. 13C NMR (100 MHz, CDCl3) $\delta 142.6,138.7,135.9,135.5,131.8,129.1,127.4,127.2$, 122.0, 109.6, 94.0, 78.3, 28.0, 23.4, 22.7, 21.0, 16.1 .

HRMS (TOF-MS ES+): [M+H]+, calculated for $\mathrm{C}_{21} \mathrm{H}_{20} \mathrm{Br}$ : 351.0743 ; found 351.0722. Chiral HPLC (CHIRALCEL IA, 99.8\% hexane $/ 0.2 \%$ IPA, $1.0 \mathrm{~mL} / \mathrm{min}, 254 \mathrm{~nm}$, tR $1=$ $13.7 \mathrm{~min}, \mathrm{tR} 2=18.8 \mathrm{~min})$ indicated 98:02 er. $[\alpha] \mathbf{D} 20=+342.44\left(\mathrm{c}=0.40, \mathrm{CH}_{2} \mathrm{Cl}_{2}\right)$.

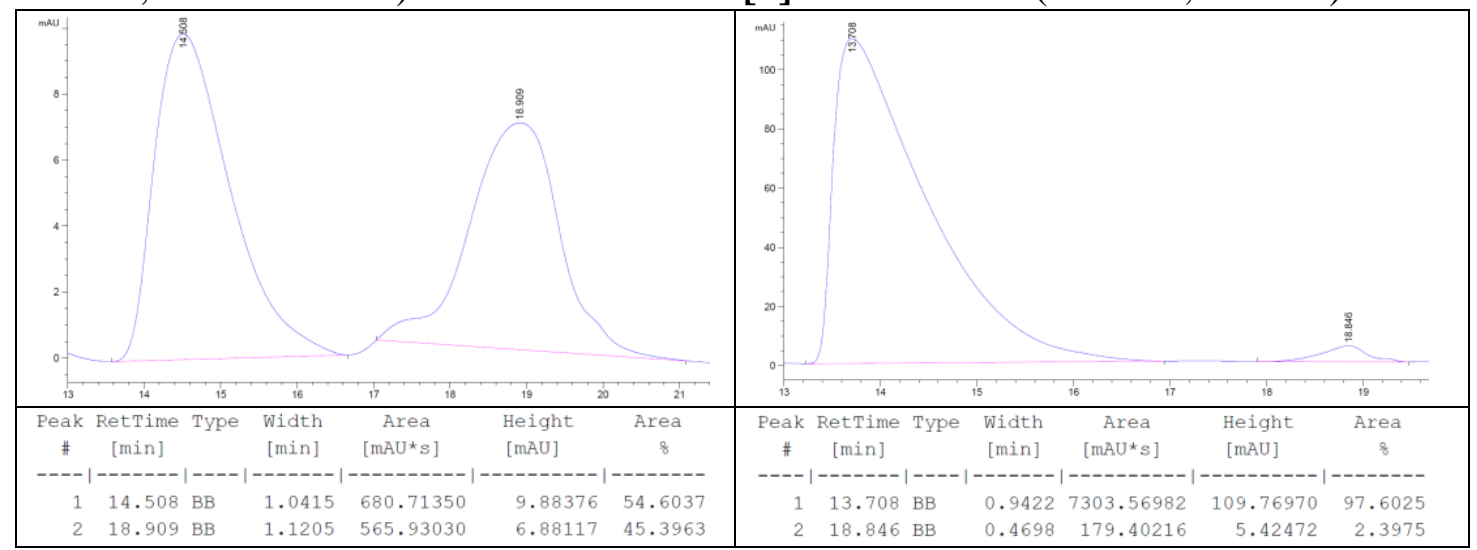

\section{1-Bromo-4-((E)-4-((1R,2R)-2-(4-chlorophenyl)-2-methylcyclopropyl)but- 1-en-3-yn-1-yl)benzene (3i)}

Alkynylated cyclopropane 3i was prepared according to the general procedure. After standard work-up and purification, $\mathbf{3 i}$ was obtained as a colorless oil $(43.1 \mathrm{mg}, 58 \%$ yield).

1H NMR (400 MHz, CDCl3) $\delta 7.49(\mathrm{~d}, J=8.5 \mathrm{~Hz}, 2 \mathrm{H}), 7.33-7.25(\mathrm{~m}, 6 \mathrm{H}), 6.88(\mathrm{~d}, J=$ $16.2 \mathrm{~Hz}, 1 \mathrm{H}), 6.24(\mathrm{dd}, J=2.0,16.2 \mathrm{~Hz}, 1 \mathrm{H}), 1.82-1.78(\mathrm{~m}, 1 \mathrm{H}), 1.61$ (s, 3H), 1.49 (dd, $J$ $=4.6,8.9 \mathrm{~Hz}, 1 \mathrm{H}), 1.07(\mathrm{dd}, J=4.7,5.6 \mathrm{~Hz}, 1 \mathrm{H}) .13 \mathrm{C} \mathbf{~ N M R}(\mathbf{1 0 0} \mathbf{~ M H z}, \mathbf{C D C l}) \delta 144.0$, 138.9, 135.4, 132.0, 131.8, 128.6, 128.5, 127.4, 122.1, 109.4, 93.3, 78.7, 27.7, 23.3, 22.5, 16.4. HRMS (TOF-MS ES+): [M+H]+, calculated for $\mathrm{C}_{20} \mathrm{H}_{17} \mathrm{BrCl}$ : 371.0197; found 371.0166.

Chiral HPLC (CHIRALCEL IC, 99.8\% hexane $/ 0.2 \%$ IPA, $1.0 \mathrm{~mL} / \mathrm{min}, 254 \mathrm{~nm}$, tR1 = $8.0 \mathrm{~min}, \mathrm{tR} 2=8.3 \mathrm{~min})$ indicated $97: 03 \mathrm{er} .[\alpha]_{\mathrm{D} 20}=+269.24\left(\mathrm{c}=1.68, \mathrm{CH}_{2} \mathrm{Cl}_{2}\right)$.

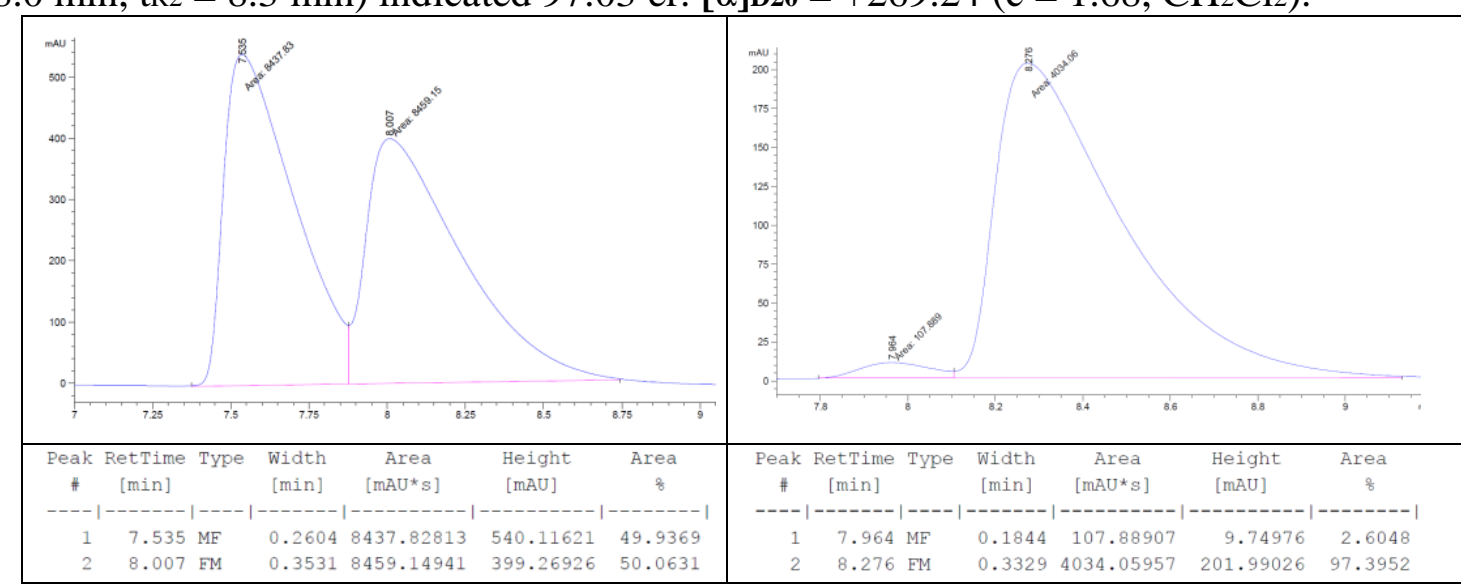




\section{1-Bromo-4-((E)-4-((1R,2R)-2-(4-methoxyphenyl)-2- methylcyclopropyl)but-1-en-3-yn-1-yl)benzene (3j)}

Alkynylated cyclopropane $\mathbf{3 j}$ was prepared according to the general procedure. After standard work-up and purification, 3j was obtained as a colorless oil (36.5 mg, 50\% yield).

1H NMR (400 MHz, CDCl3) $\delta 7.48$ - 7.44 (m, 2H), 7.29 - 7.22 (m, 4H), 6.90 - 6.81 (m, $3 \mathrm{H}), 6.22(\mathrm{dd}, J=16.2,2.1 \mathrm{~Hz}, 1 \mathrm{H}), 3.82(\mathrm{~s}, 3 \mathrm{H}), 1.76(\mathrm{ddd}, J=8.9,5.6,2.0 \mathrm{~Hz}, 1 \mathrm{H})$, $1.58(\mathrm{~s}, 3 \mathrm{H}), 1.46(\mathrm{dd}, J=8.9,4.4 \mathrm{~Hz}, 1 \mathrm{H}), 1.00(\mathrm{dd}, J=5.6,4.4 \mathrm{~Hz}, 1 \mathrm{H}) .13 \mathrm{C} \mathrm{NMR}$ (100 MHz, CDCl3) $\delta 144.0,138.9,135.4,132.0,131.8,128.6,128.5,127.4,122.1$, 109.4, 93.3, 78.7, 27.7, 23.3, 22.5, 16.4. HRMS (TOF-MS ES+): [M+H]+, calculated for $\mathrm{C}_{21} \mathrm{H}_{19} \mathrm{BrO}: 366.0619$; found 366.0628.

Chiral HPLC (CHIRALCEL ADH, 99\% hexane/1\%IPA, $1.0 \mathrm{~mL} / \mathrm{min}, 254 \mathrm{~nm}$, tR1 = $\left.21.8 \mathrm{~min}, \mathrm{tR}_{2}=23.5 \mathrm{~min}\right)$ indicated 94:06 er. $[\alpha]_{\mathbf{D} 20}=+266.37\left(\mathrm{c}=0.20, \mathrm{CH}_{2} \mathrm{Cl}_{2}\right)$.

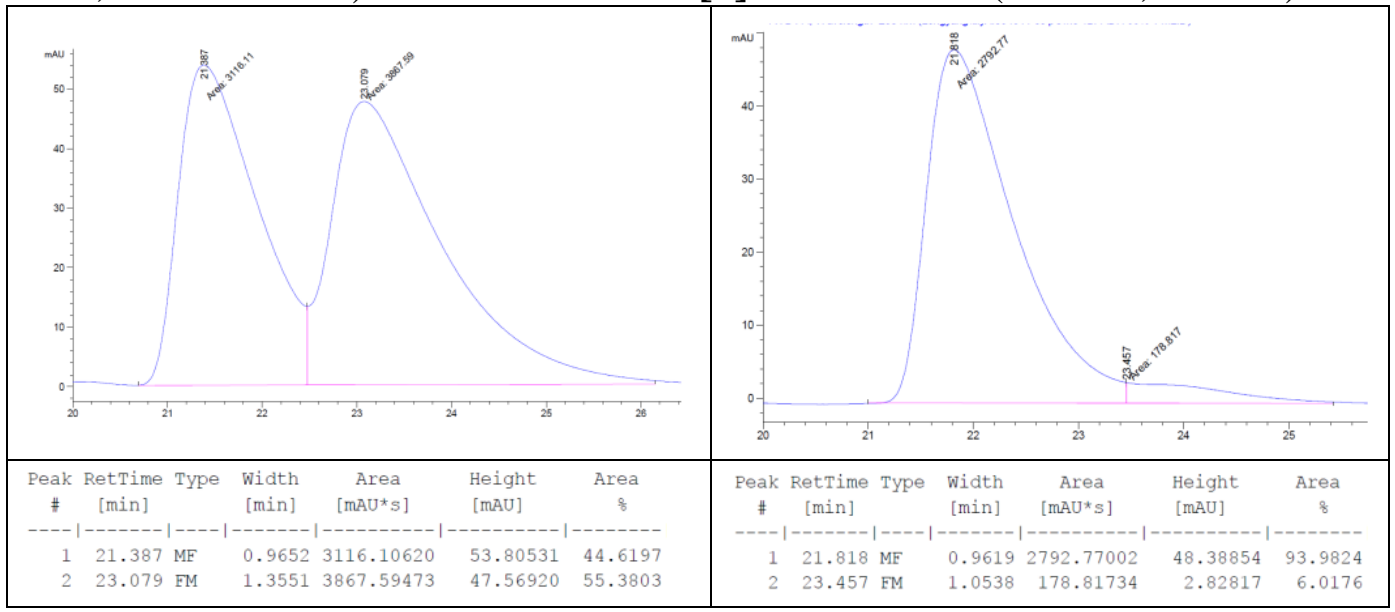

\section{1-((E)-4-((1R,2R)-2-(4-Chlorophenyl)-2-methylcyclopropyl)but-1-en-3-}

\section{yn-1-yl)naphthalene (3k)}

Alkynylated cyclopropane 3k was prepared according to the general procedure. After standard work-up and purification, 3k was obtained as a colorless oil (40.4 mg, 59\% yield).

1H NMR (400 MHz, CDCl3) $\delta$ 7.73-7.60 (m, 4H), 7.50-7.45 (m, 1H), 7.40-7.33 (m, 2H), 7.20-7.12 (m, 4H), $7.00(\mathrm{~d}, J=16.2 \mathrm{~Hz}, 1 \mathrm{H}), 6.23(\mathrm{dd}, J=2.0,16.2 \mathrm{~Hz}, 1 \mathrm{H}), 1.72-1.66$ $(\mathrm{m}, 1 \mathrm{H}), 1.50(\mathrm{~s}, 3 \mathrm{H}), 1.36(\mathrm{dd}, J=4.5,9.0 \mathrm{~Hz}, 1 \mathrm{H}), 0.95(\mathrm{dd}, J=4.8,5.5 \mathrm{~Hz}, 1 \mathrm{H}) .{ }_{13} \mathrm{C}$ NMR (100 MHz, CDCl3) $\delta 144.1,140.4,134.0,133.5,133.3,132.0,128.7,128.5,128.4$, 128.1, 127.7, 126.4, 126.4, 126.2, 122.7, 108.9, 92.9, 79.1, 27.7, 23.4, 22.5, 16.5. HRMS (TOF-MS ES+): [M+H]+, calculated for $\mathrm{C}_{24} \mathrm{H}_{20} \mathrm{Cl}$ : 343.1248 ; found 343.1277 .

Chiral HPLC (CHIRALCEL ADH, 99.8\% hexane/0.2\% IPA, $1.0 \mathrm{~mL} / \mathrm{min}, 254 \mathrm{~nm}$, tR1 = $39.9 \mathrm{~min}, \mathrm{tR} 2=49.1 \mathrm{~min})$ indicated $>99: 01 \mathrm{er} .[\alpha] \mathbf{D 2 0}=+256.85\left(\mathrm{c}=0.23, \mathrm{CH}_{2} \mathrm{Cl}_{2}\right)$. 


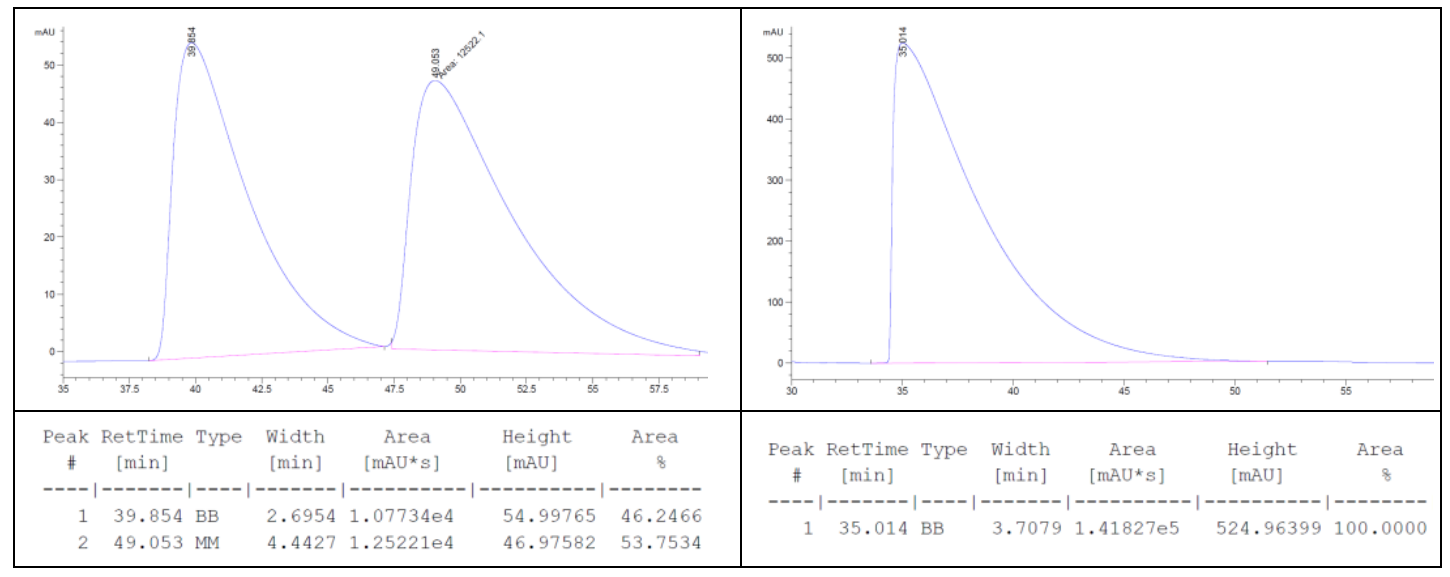

\section{Reference}

1. Tenaglia, A.; Jeune, K. L.; Giordano, L.; Buono, G. Palladium-Catalyzed Addition of Alkynes to Cyclopropenes: an Entry to Stereodefined Alkynylcyclopropanes. Org. Lett. 2011, 13, 636-639.

2. Teng, H.-L.; Ma, Y.; Zhan, G.; Nishiura, M.; Hou, Z. Asymmetric C(sp)-H Addition of Terminal Alkynes to Cyclopropenes by a Chiral Gadolinium Catalyst. ACS Catal. 2018, 8, 4705-4709. 


\section{NMR spectrum}

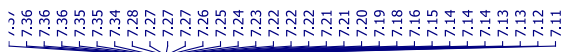
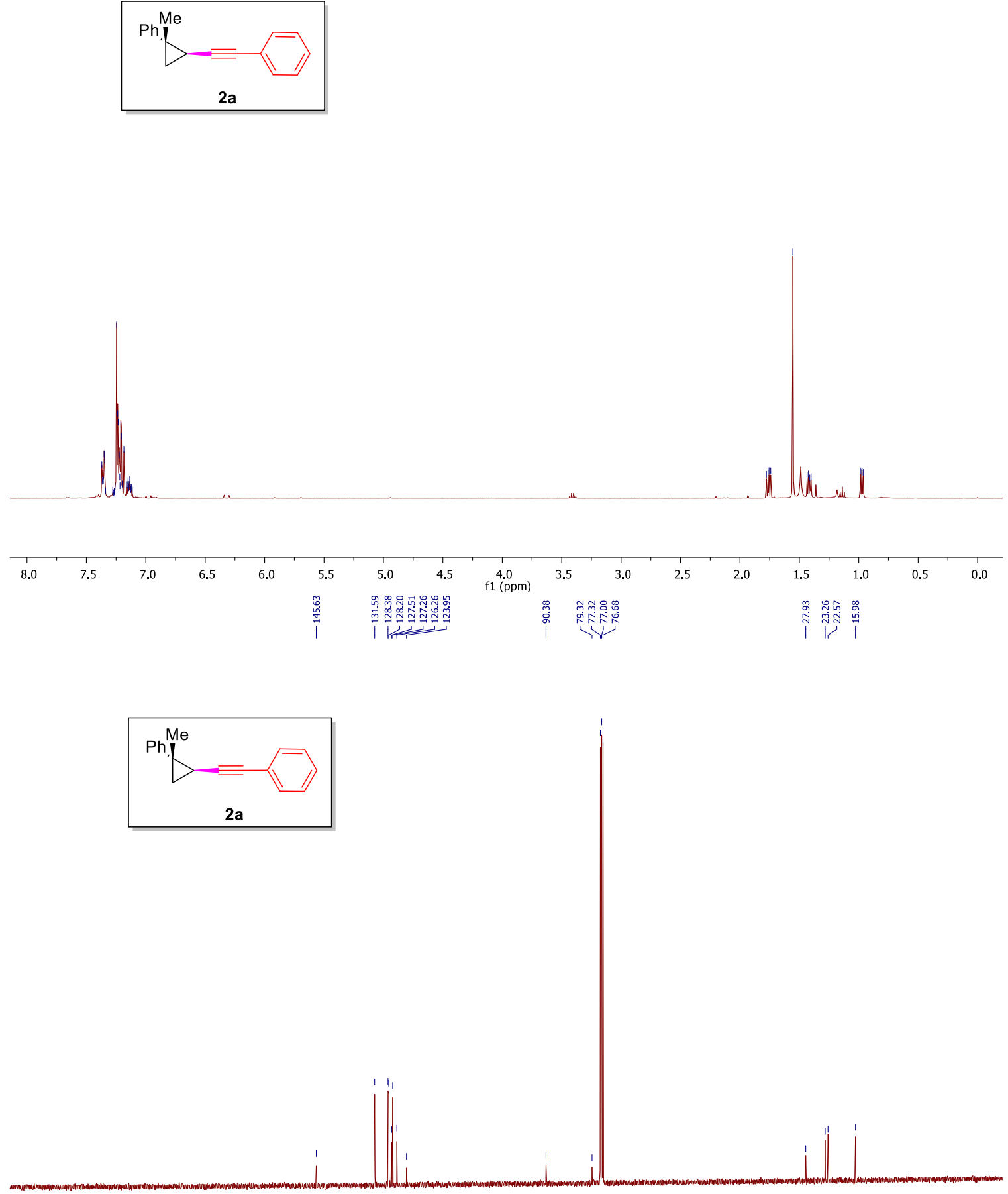
Supporting Information
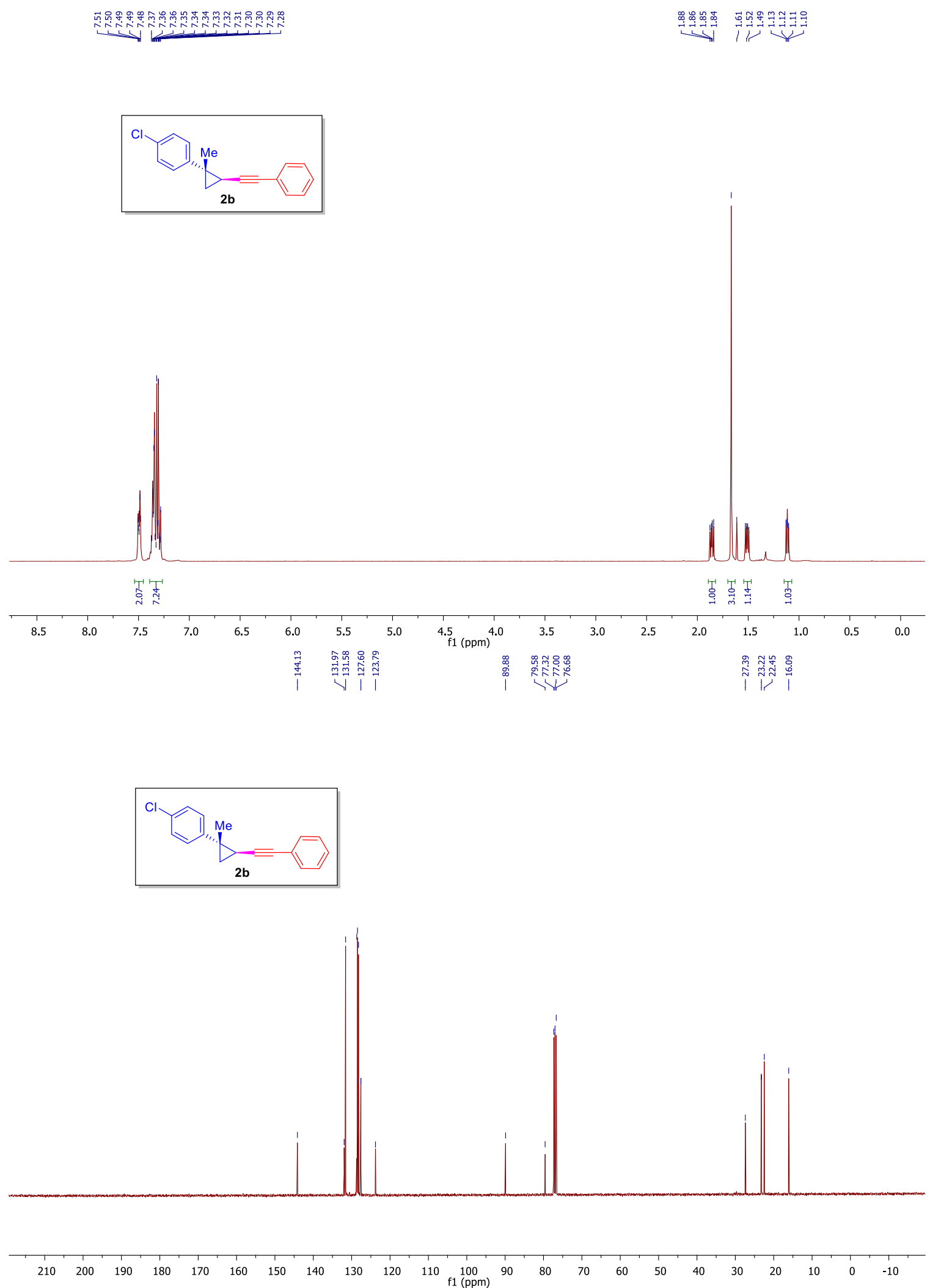
Supporting Information

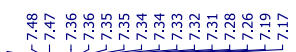

|
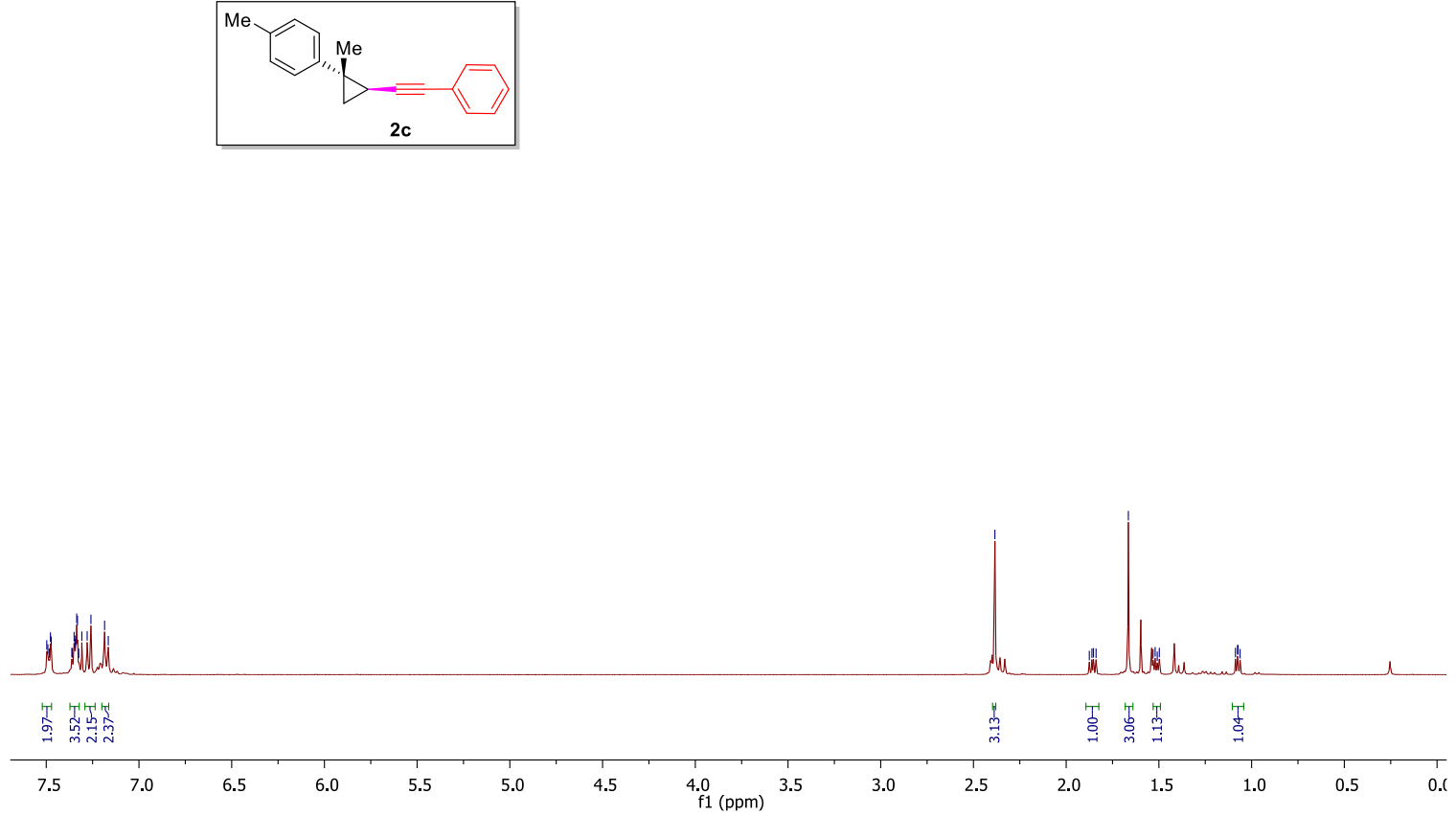

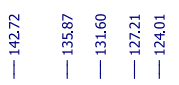

员 於

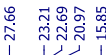
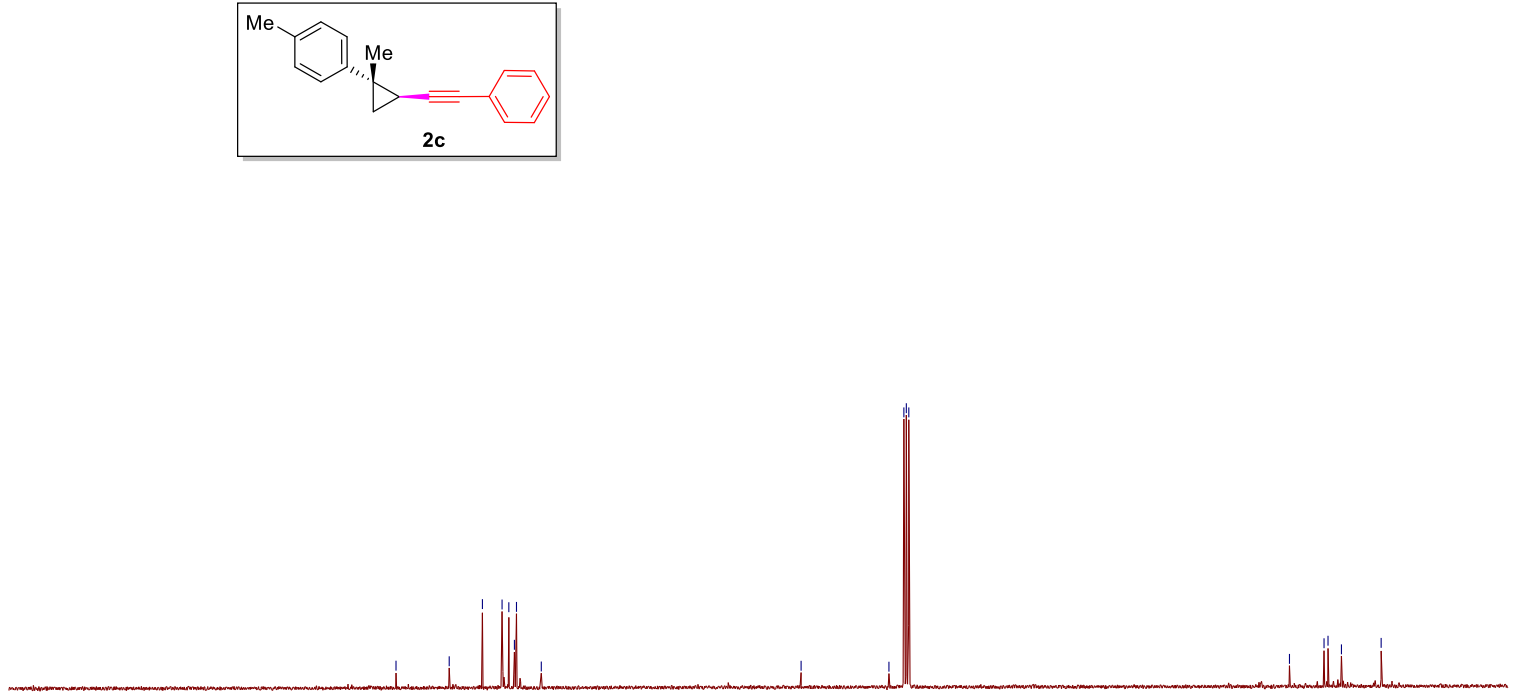
Supporting Information

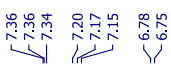

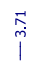

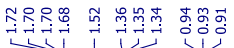
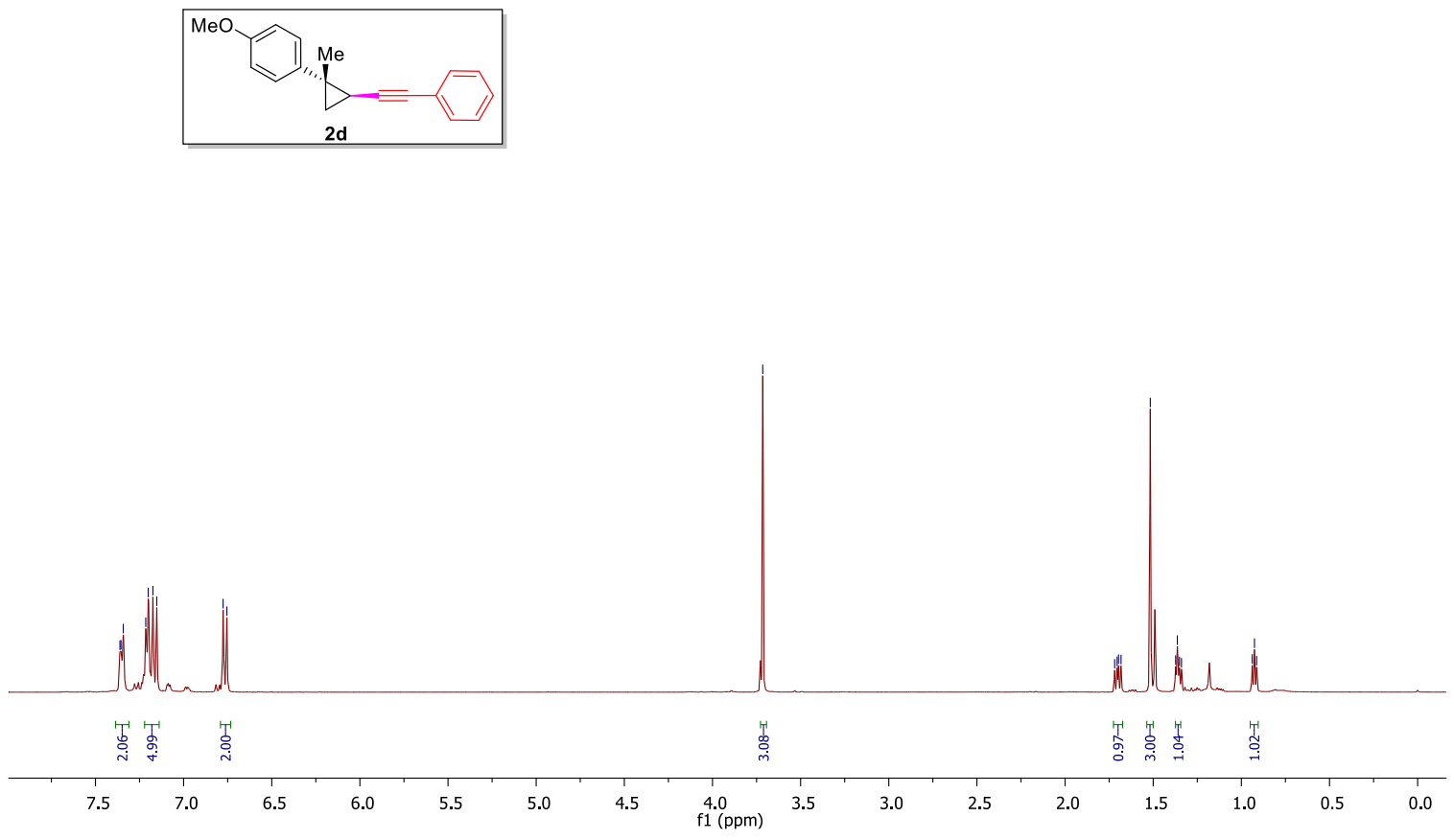

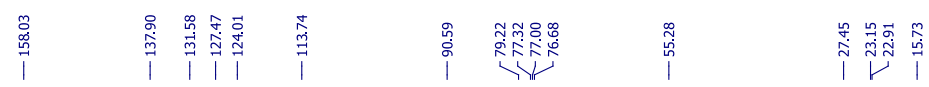
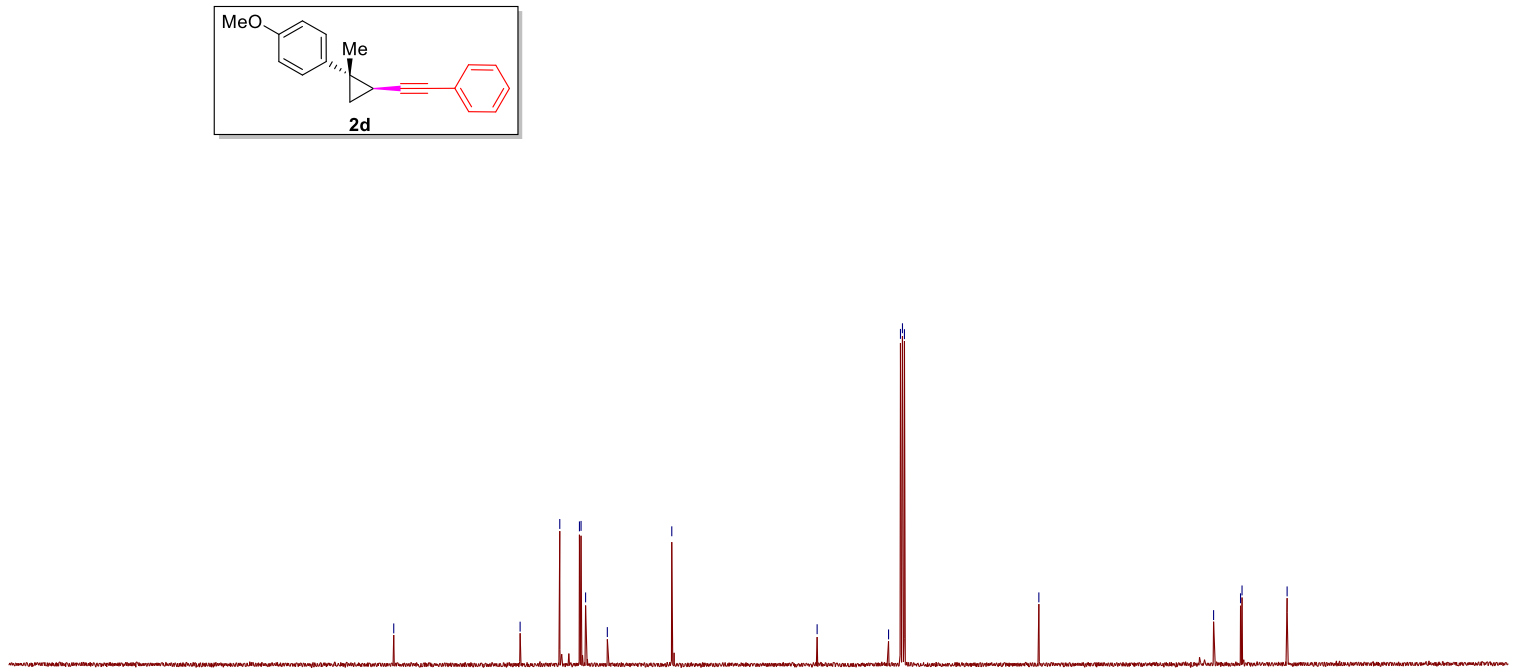
Supporting Information
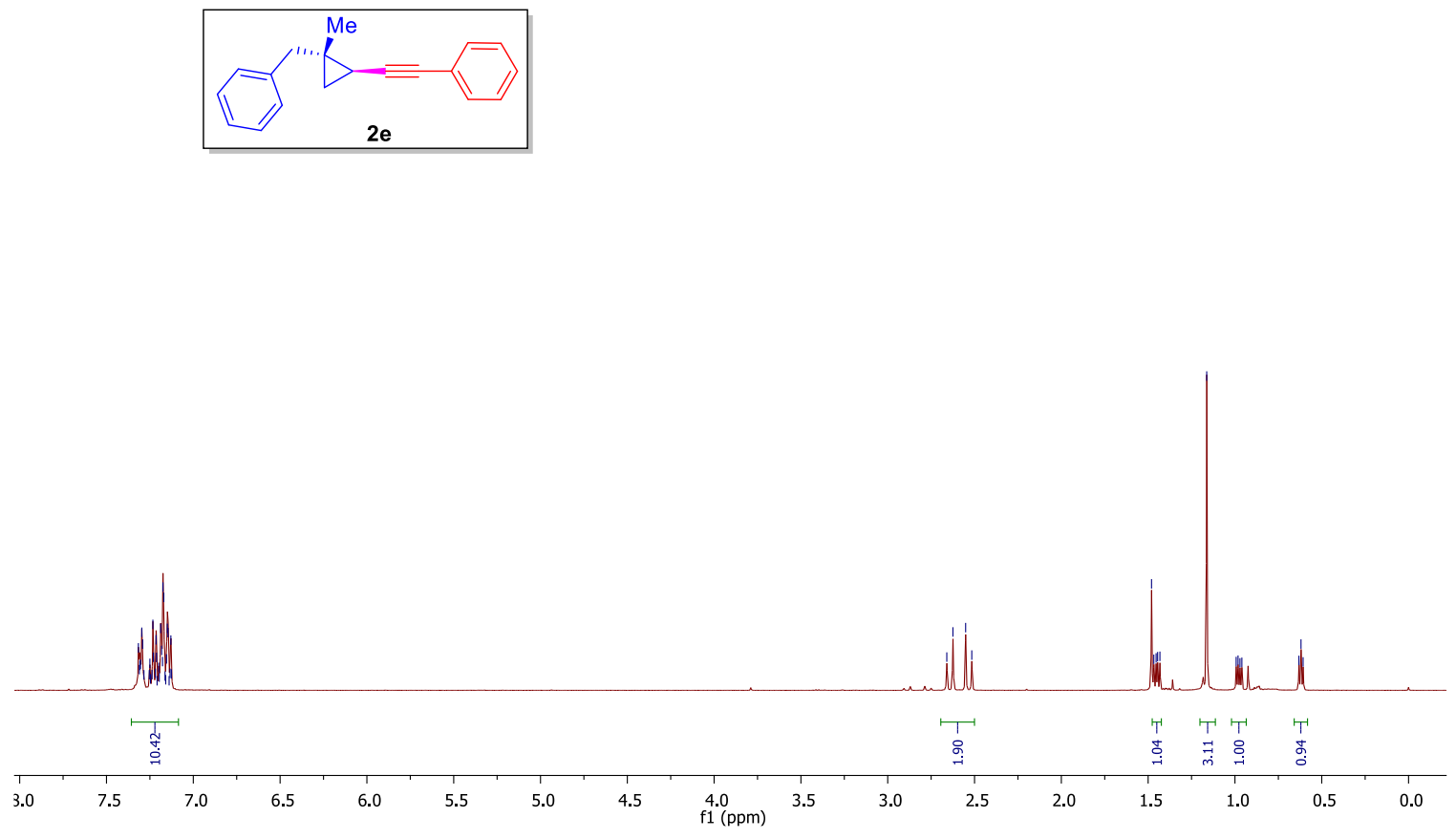

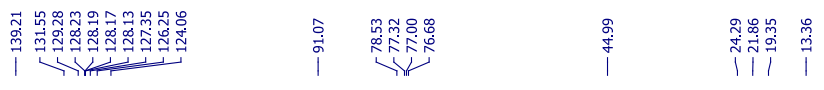
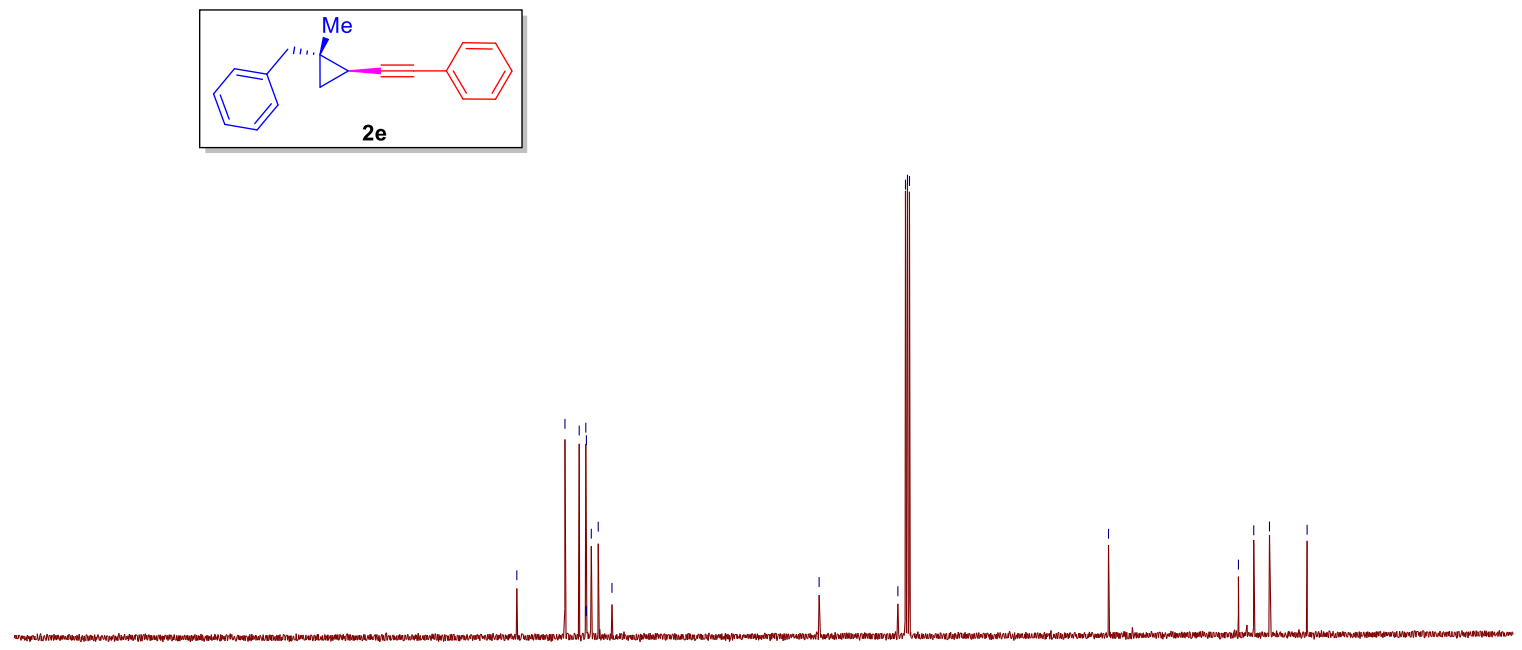
Supporting Information
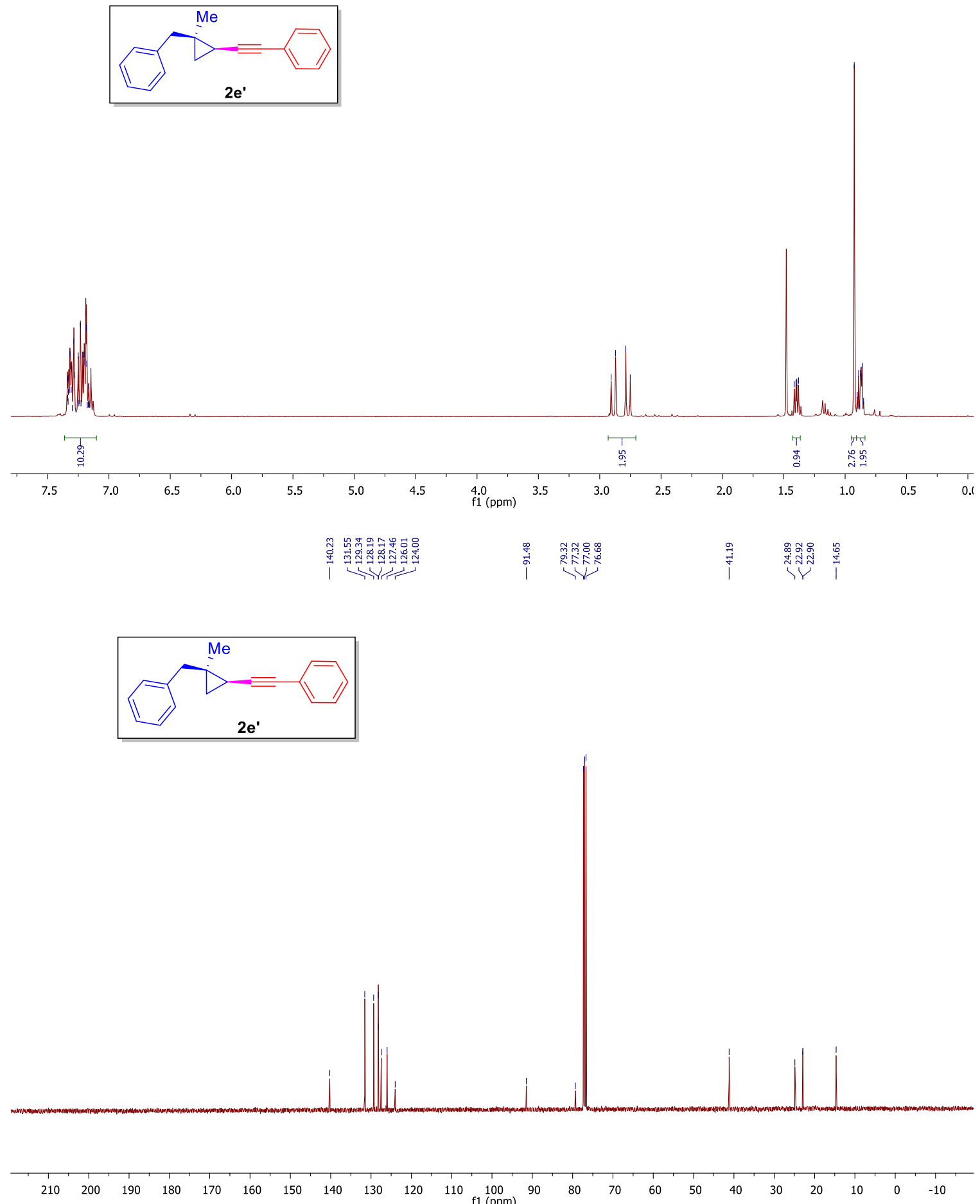
Supporting Information

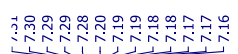

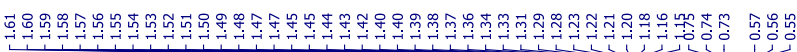
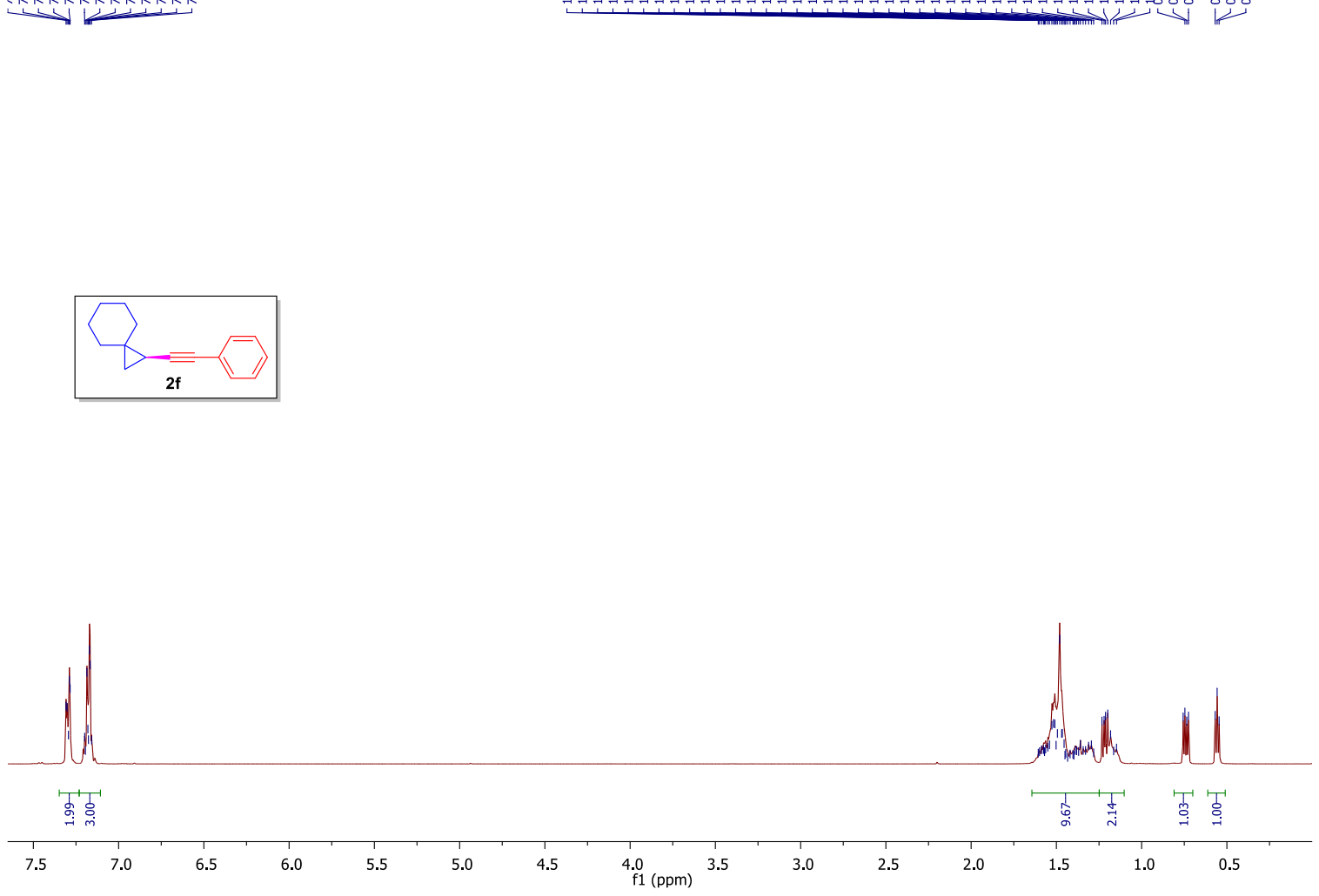

|

萡

芯唡
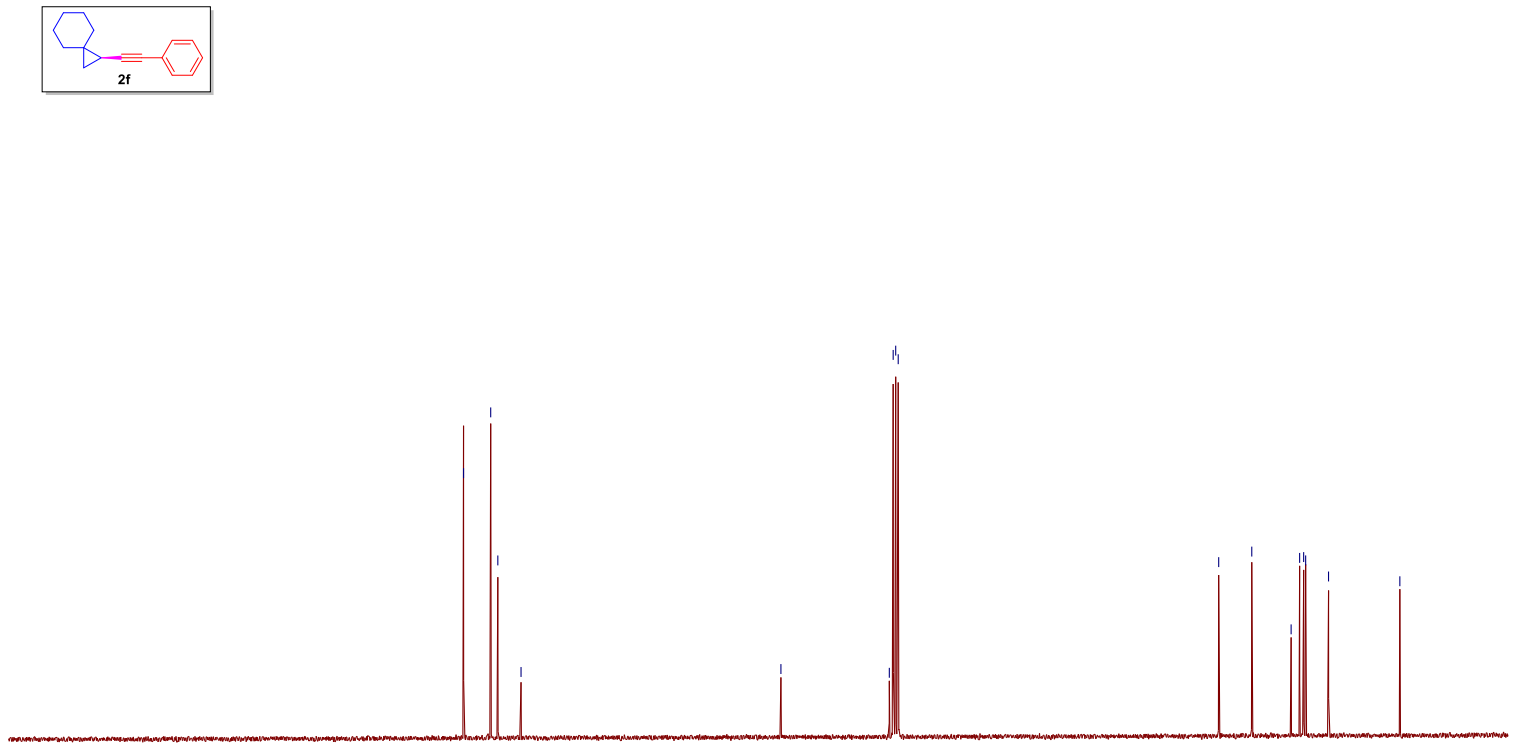
Supporting Information

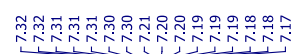

倠
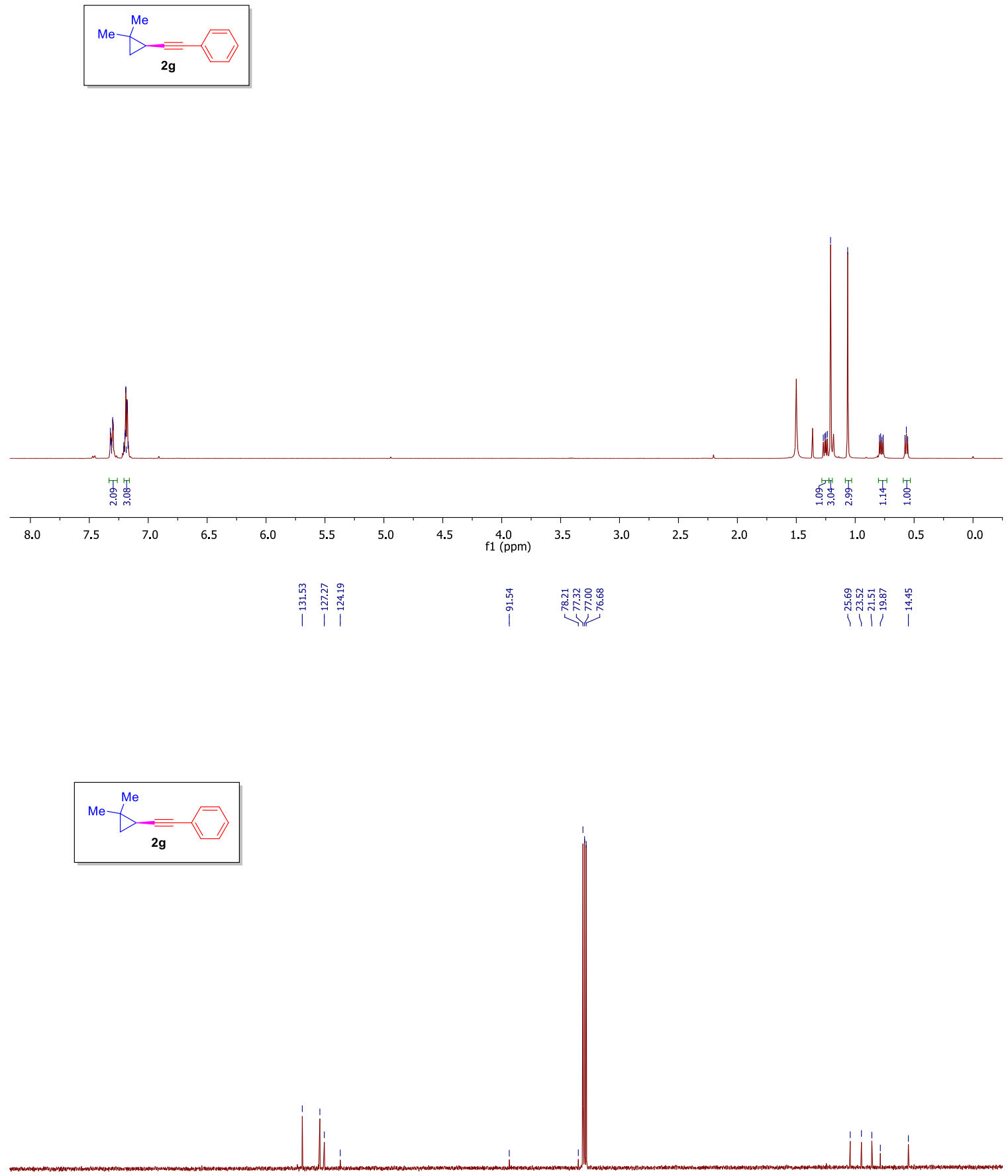
Supporting Information

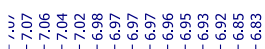

|
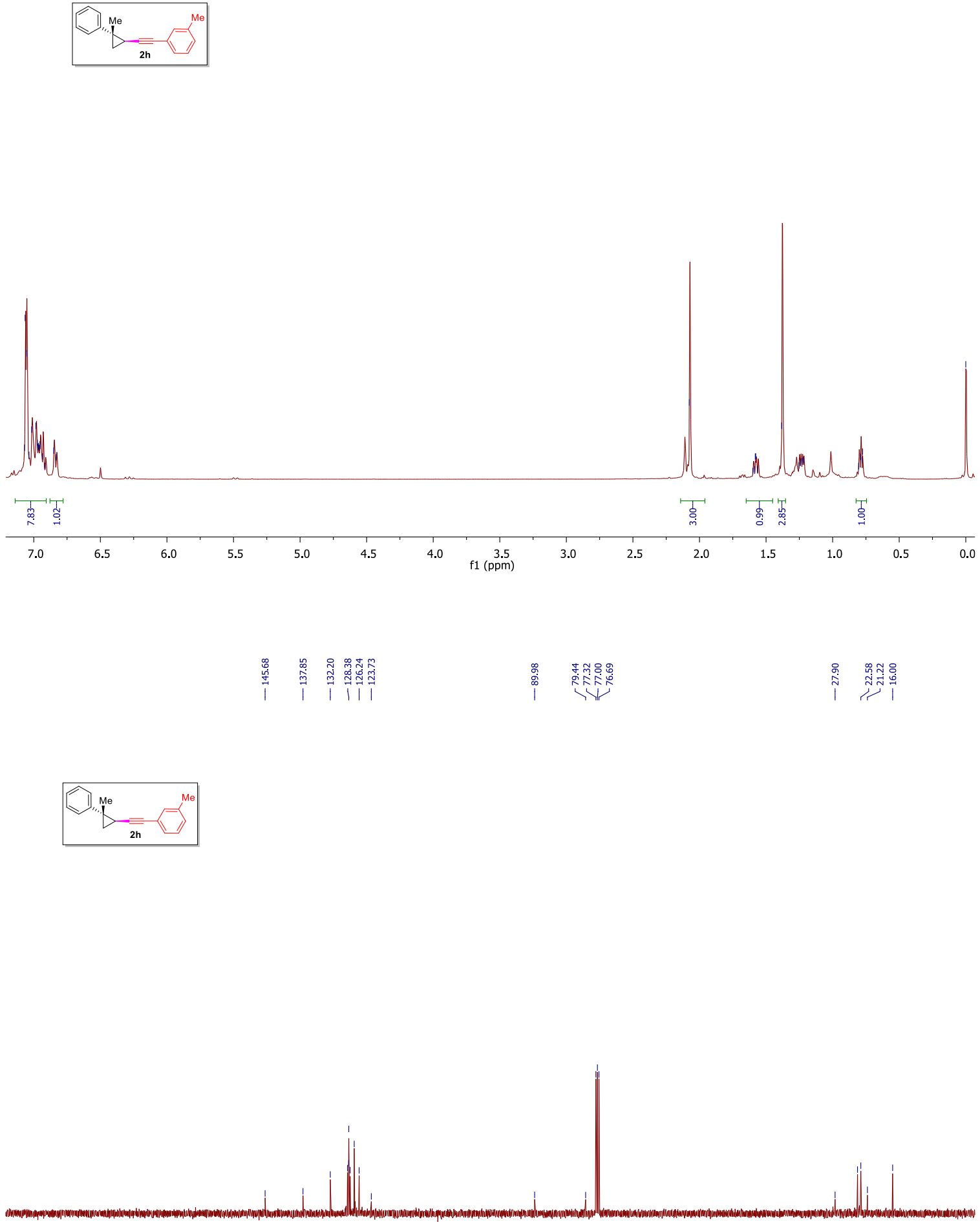

$190 \quad 180$

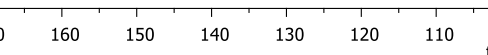

$100 \quad 90$

80


Supporting Information

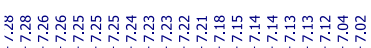

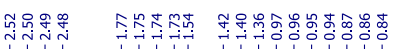
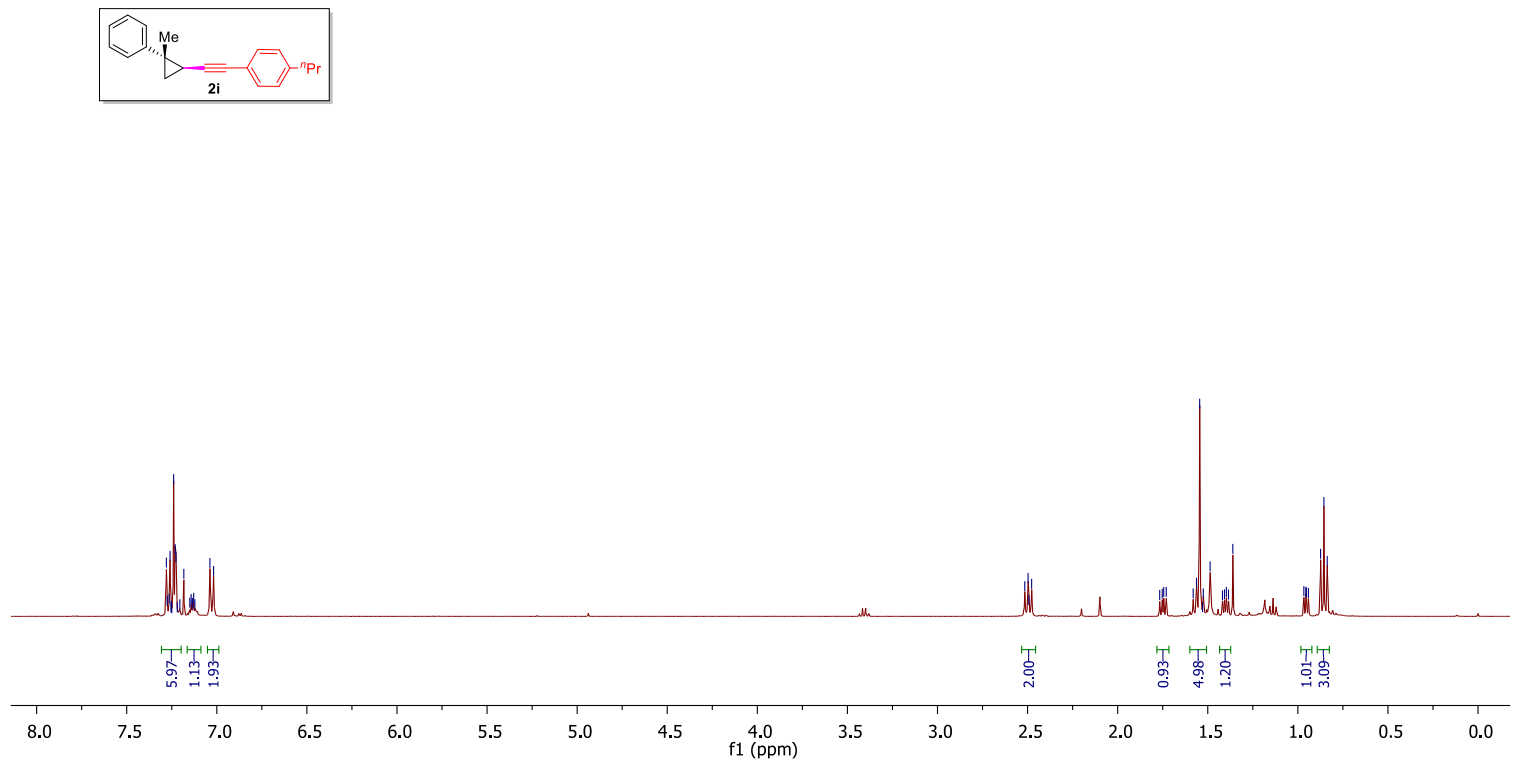

| |l|

पू

| |
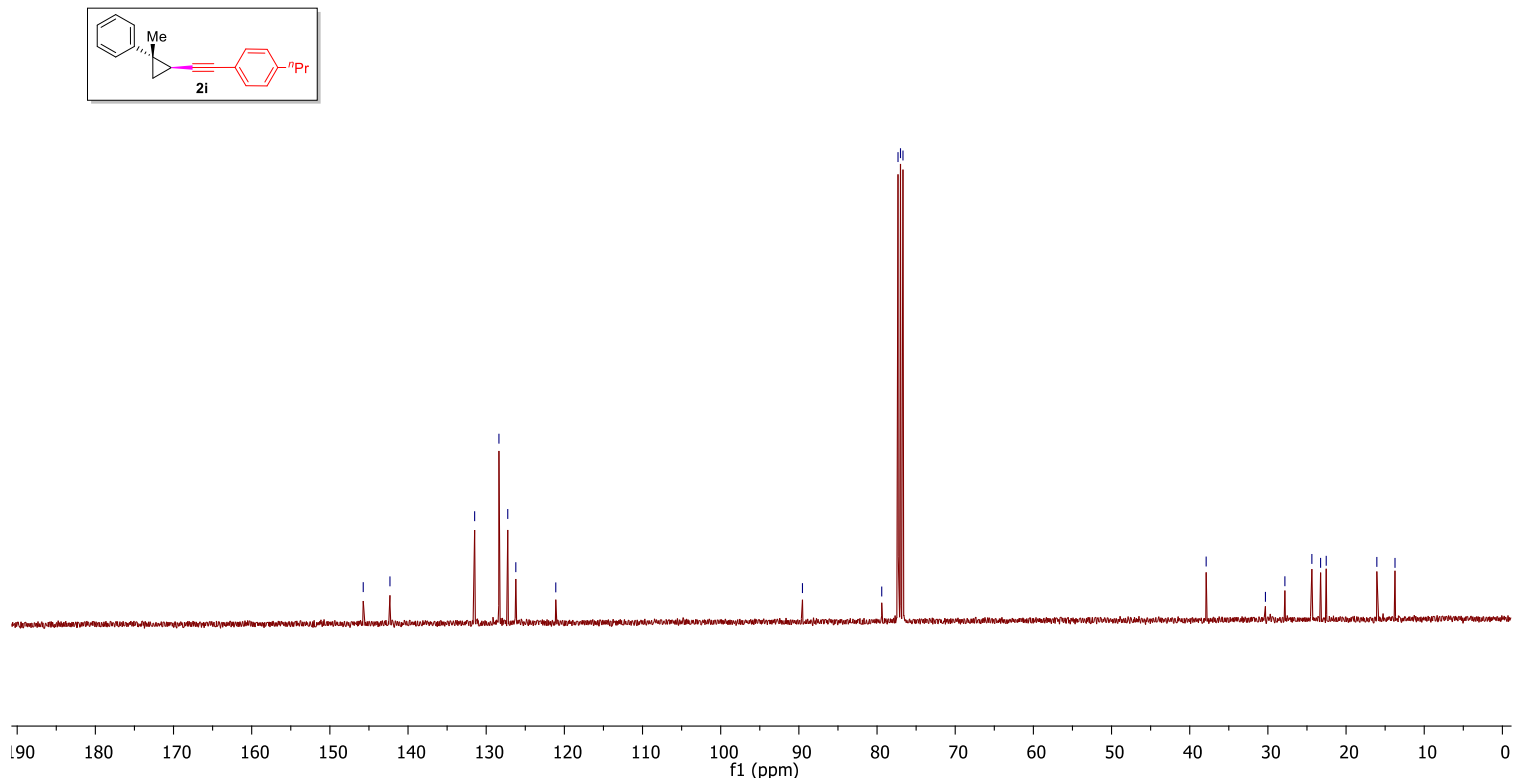
Supporting Information

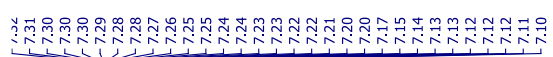

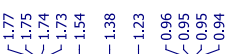

Q1, Me
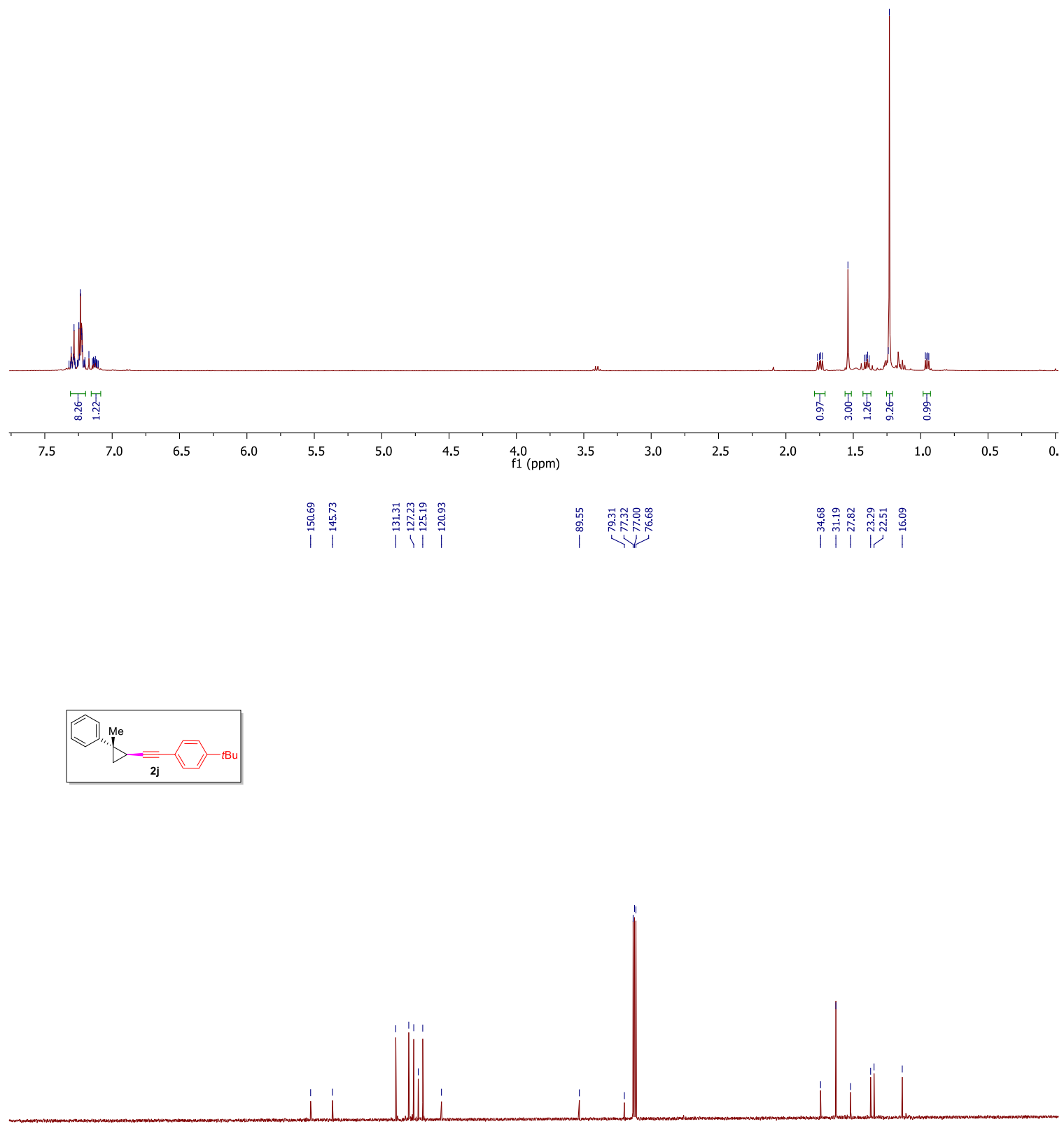
Supporting Information

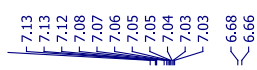
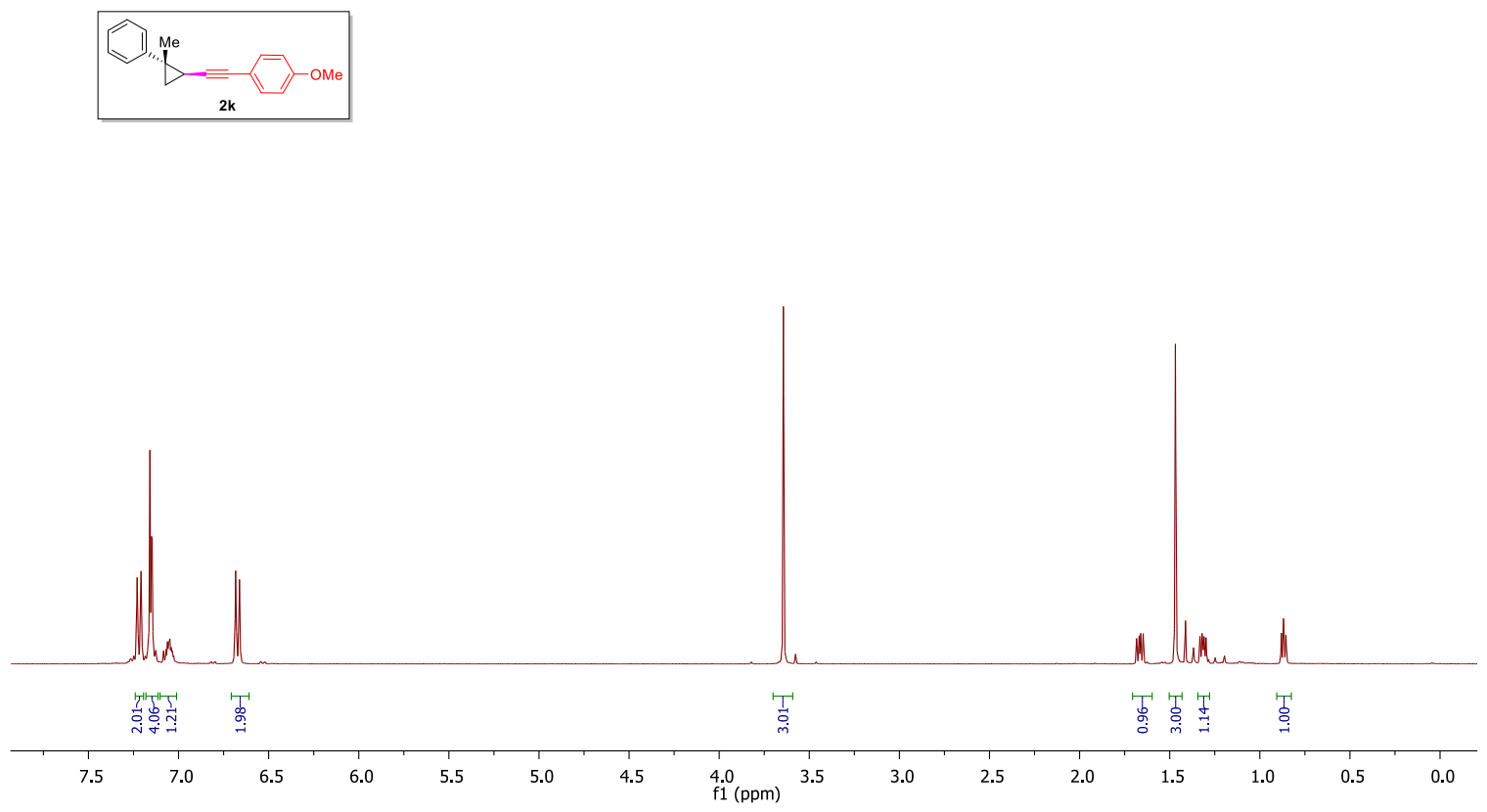

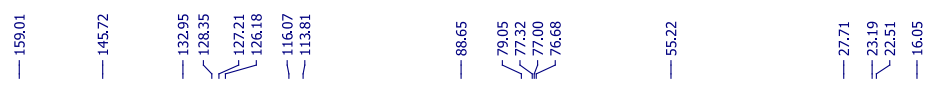
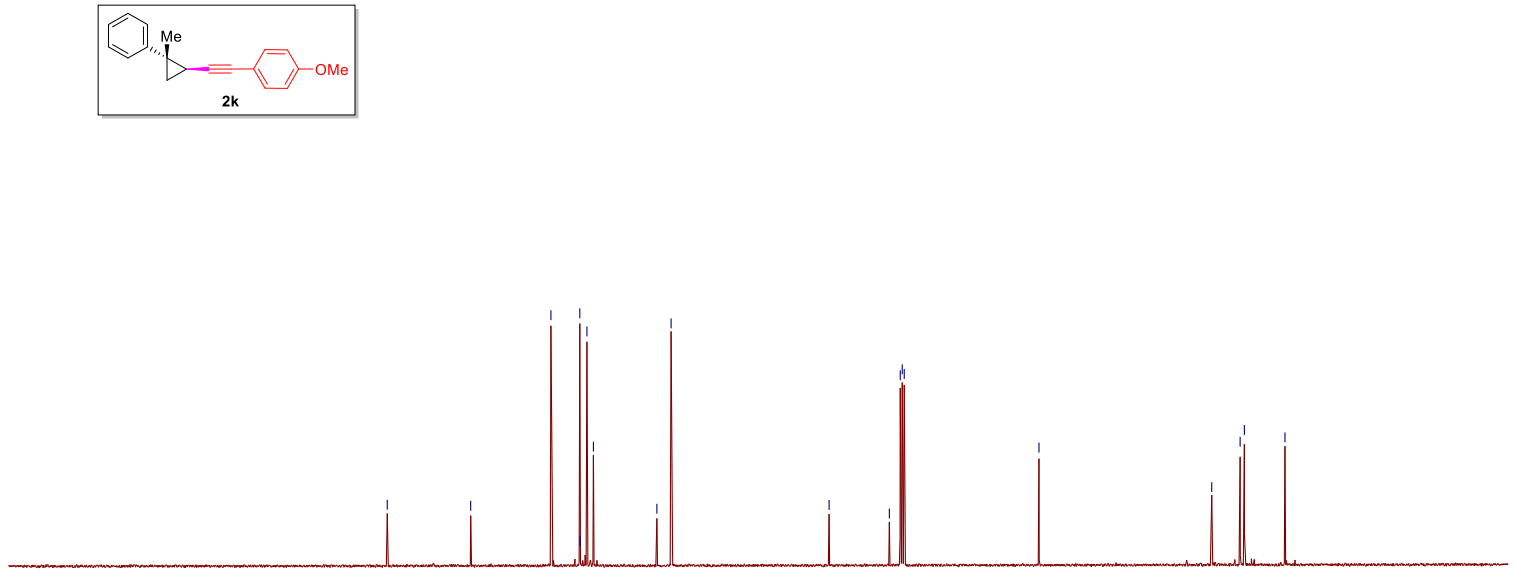
Supporting Information

\begin{tabular}{llll}
$n$ \\
\hline
\end{tabular}

$\stackrel{\infty}{\infty}$

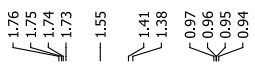
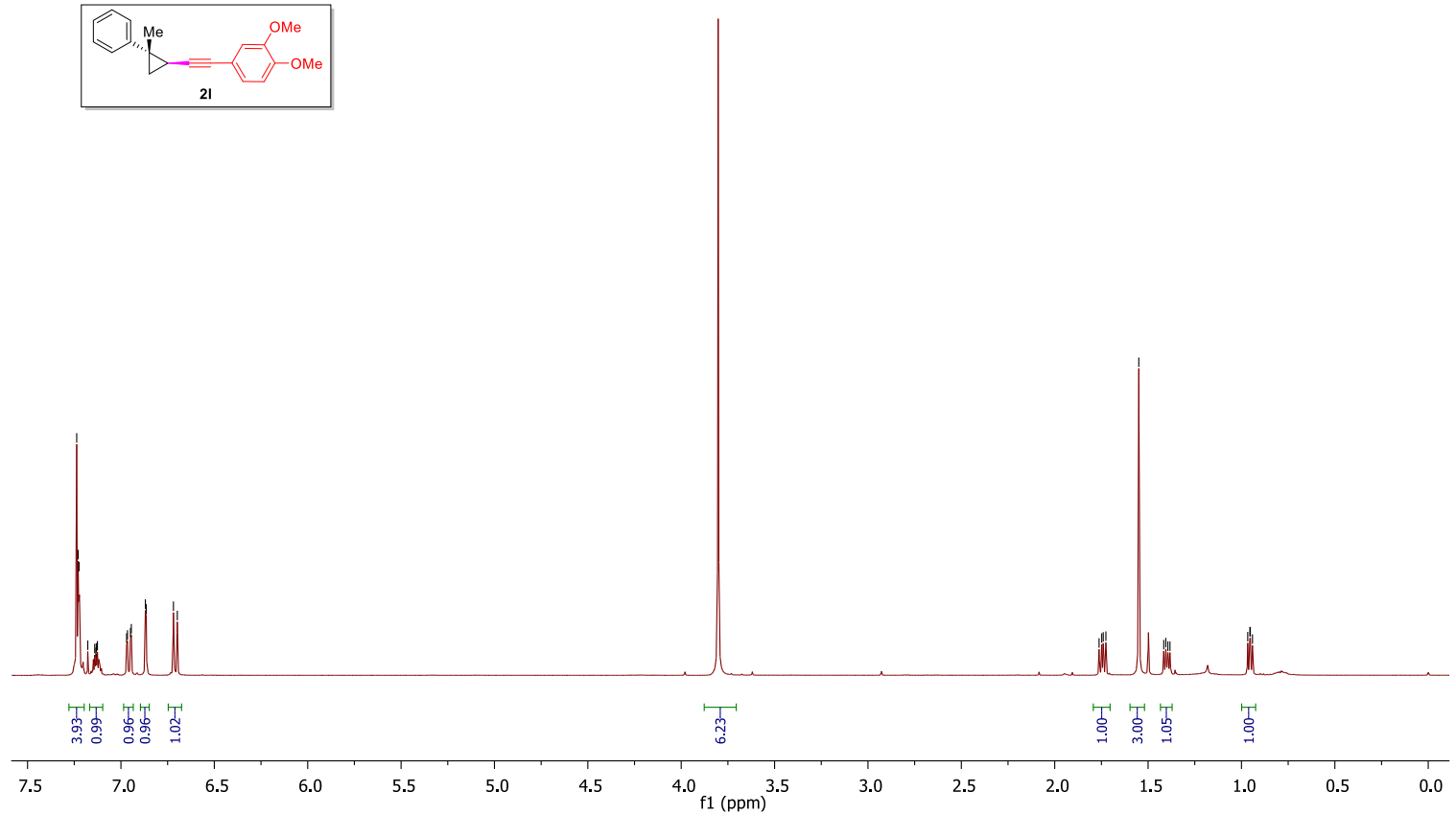

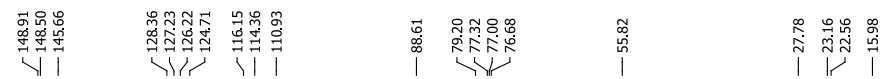
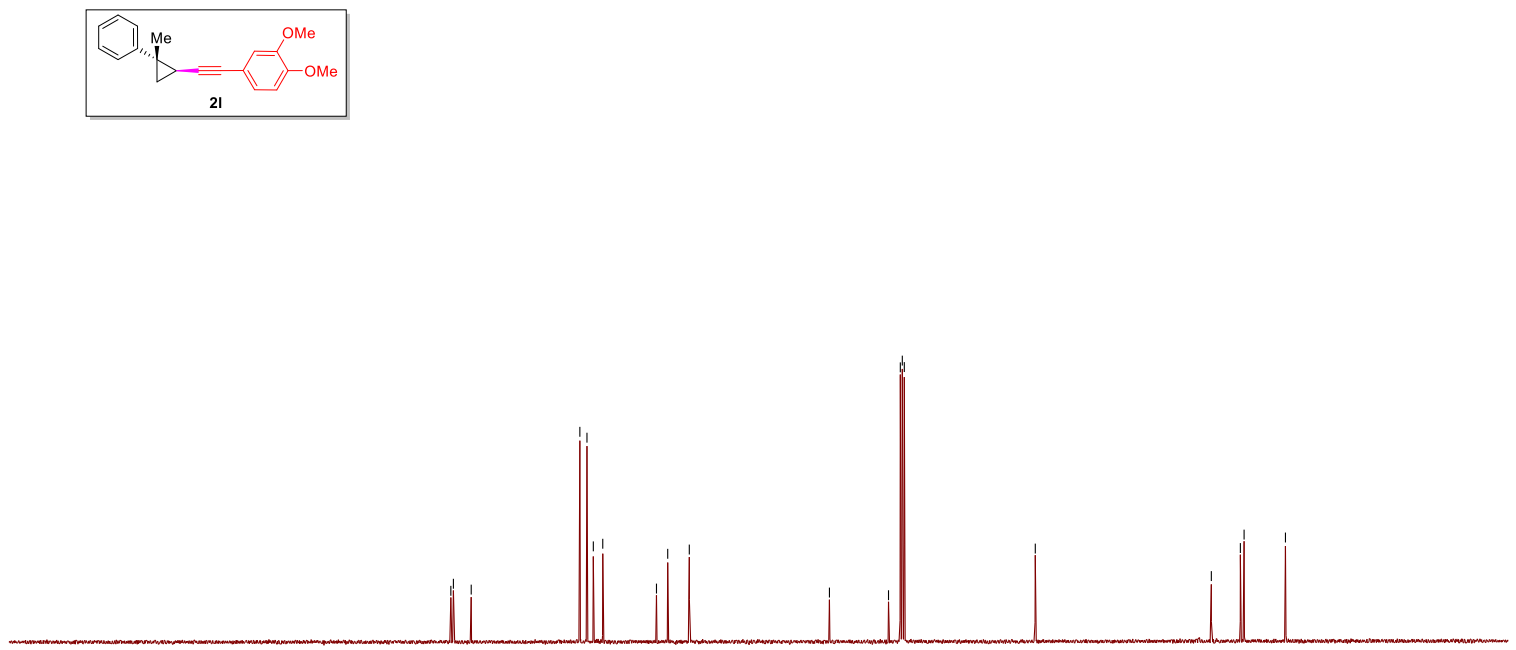
Supporting Information
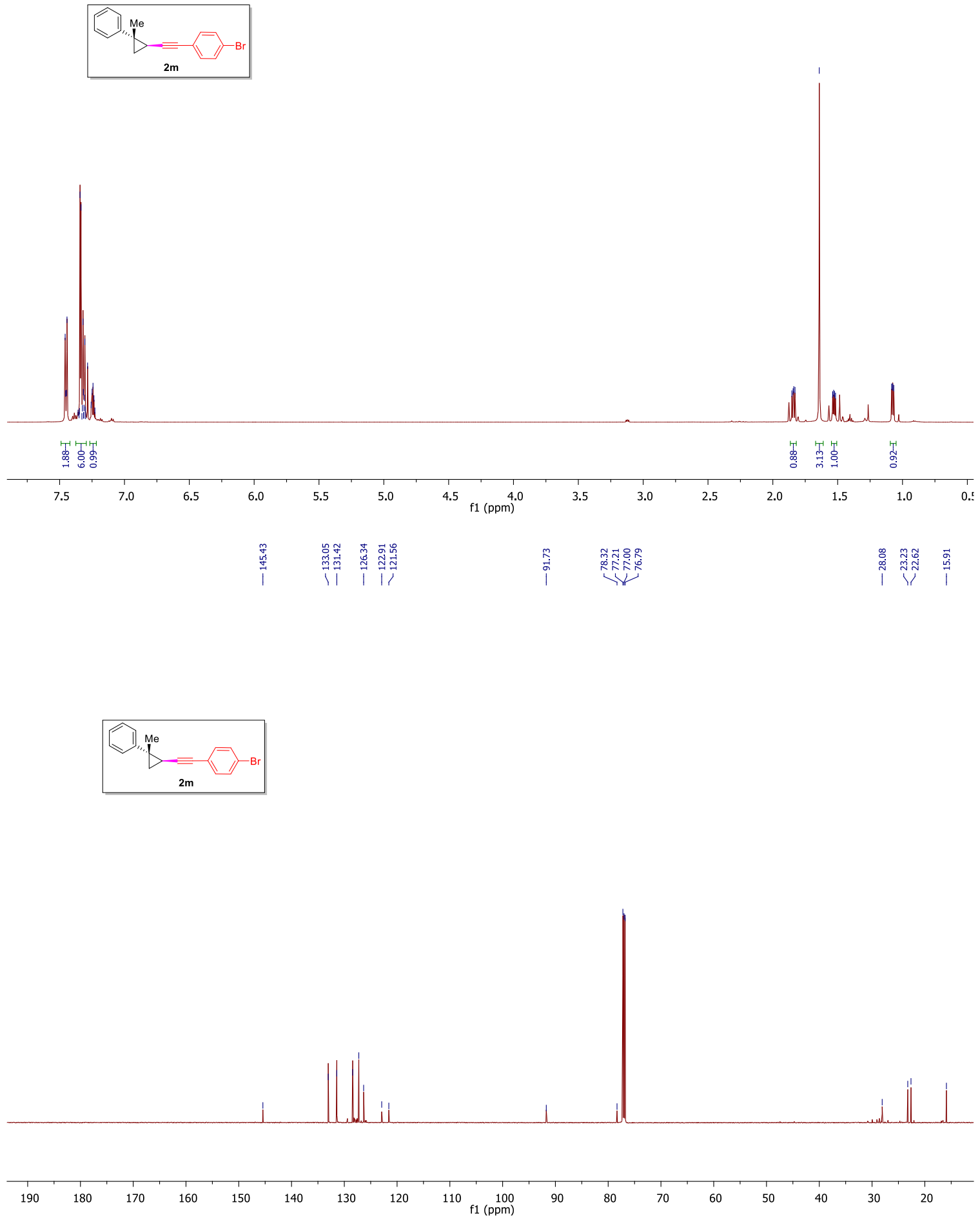
Supporting Information

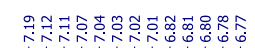
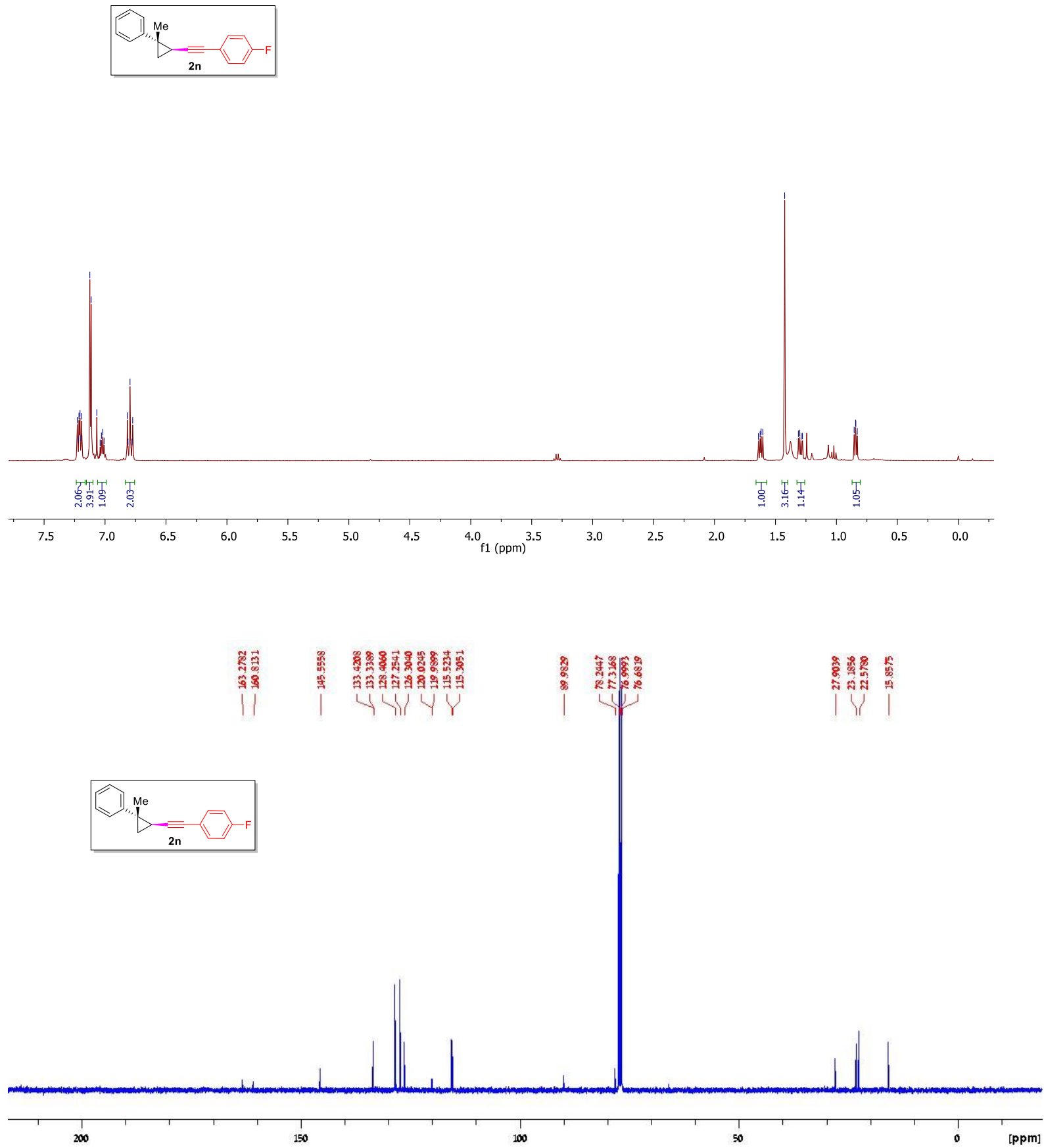

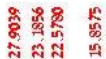

| | | 
Supporting Information

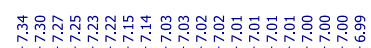

$\underbrace{2}$
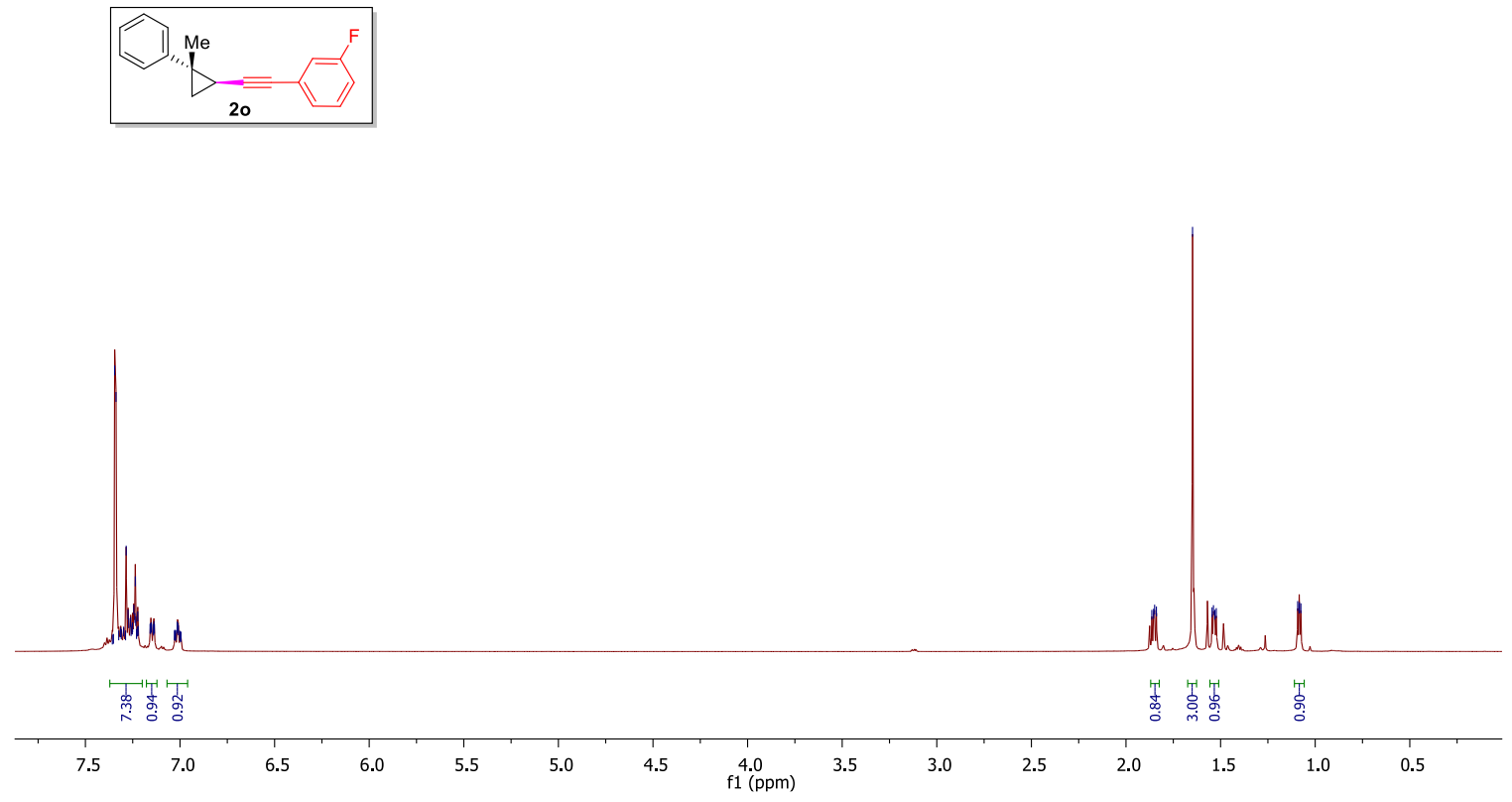

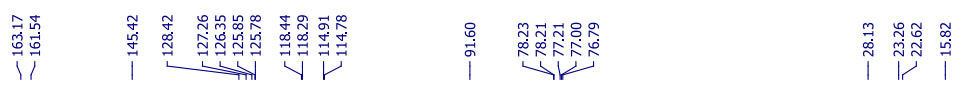
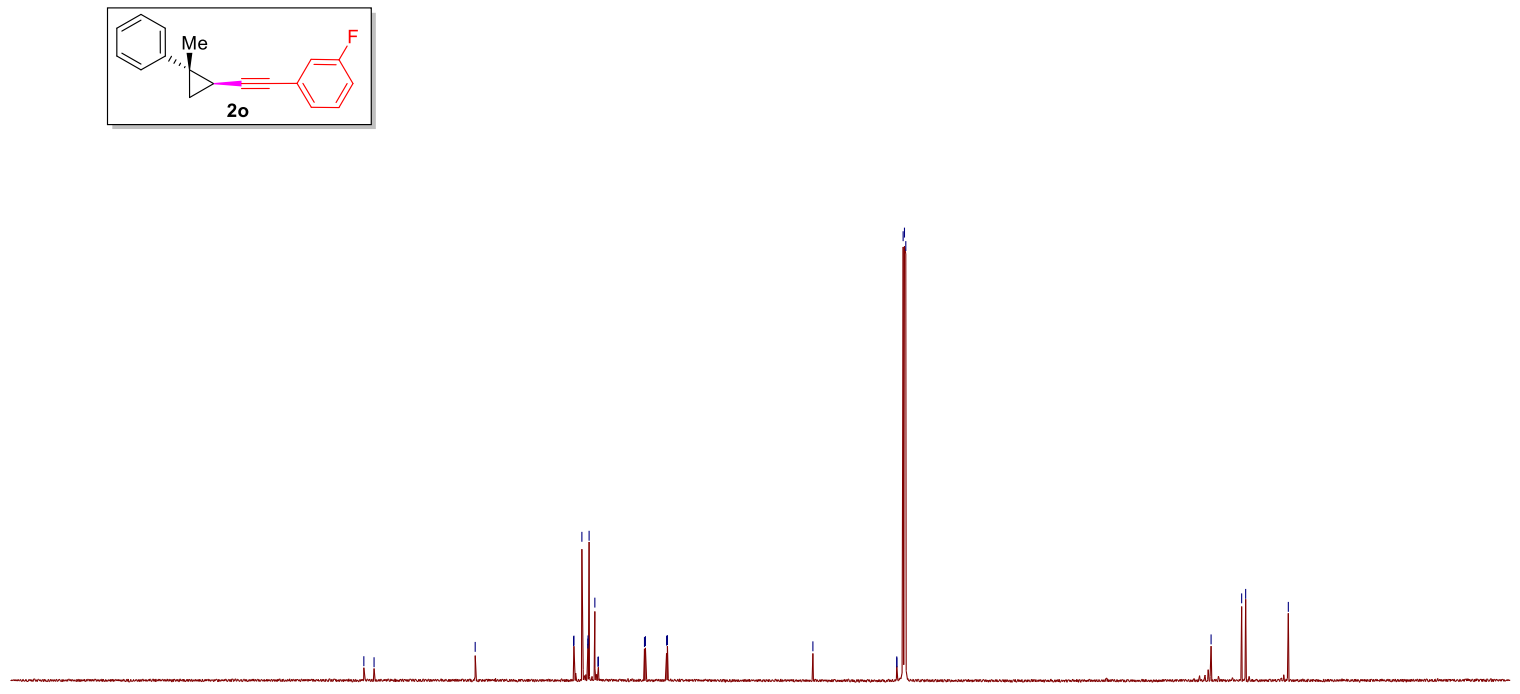
Supporting Information

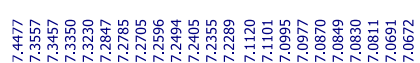

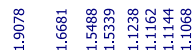

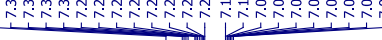

i)
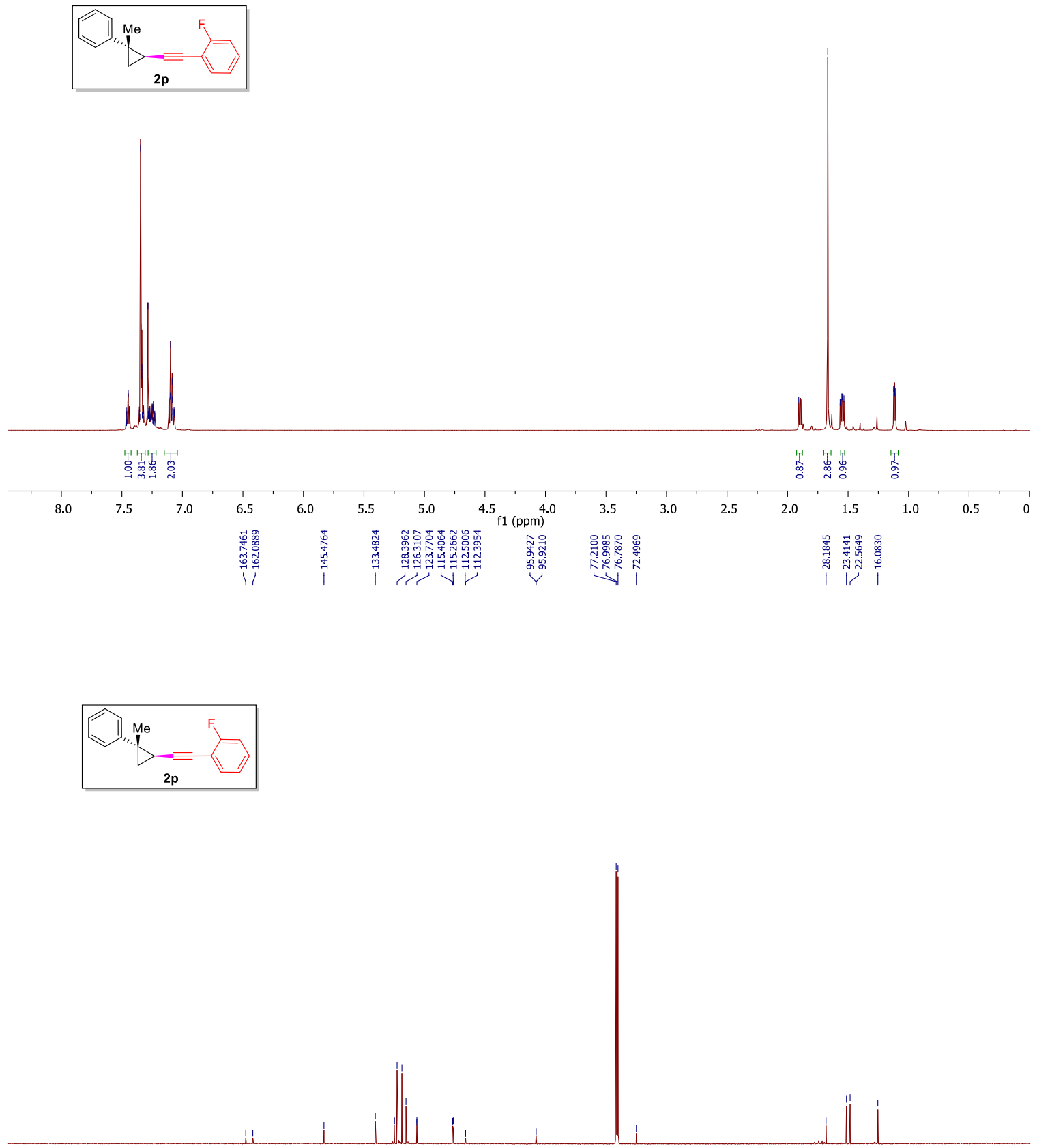

$\begin{array}{llllllllllllllllllllllllll} & 210 & 200 & 190 & 180 & 170 & 160 & 150 & 140 & 130 & 120 & 110 & 100 & 90 & 80 & 70 & 60 & 50 & 40 & 30 & 20 & 10 & 0 & -10\end{array}$ 
Supporting Information

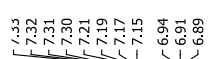

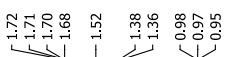
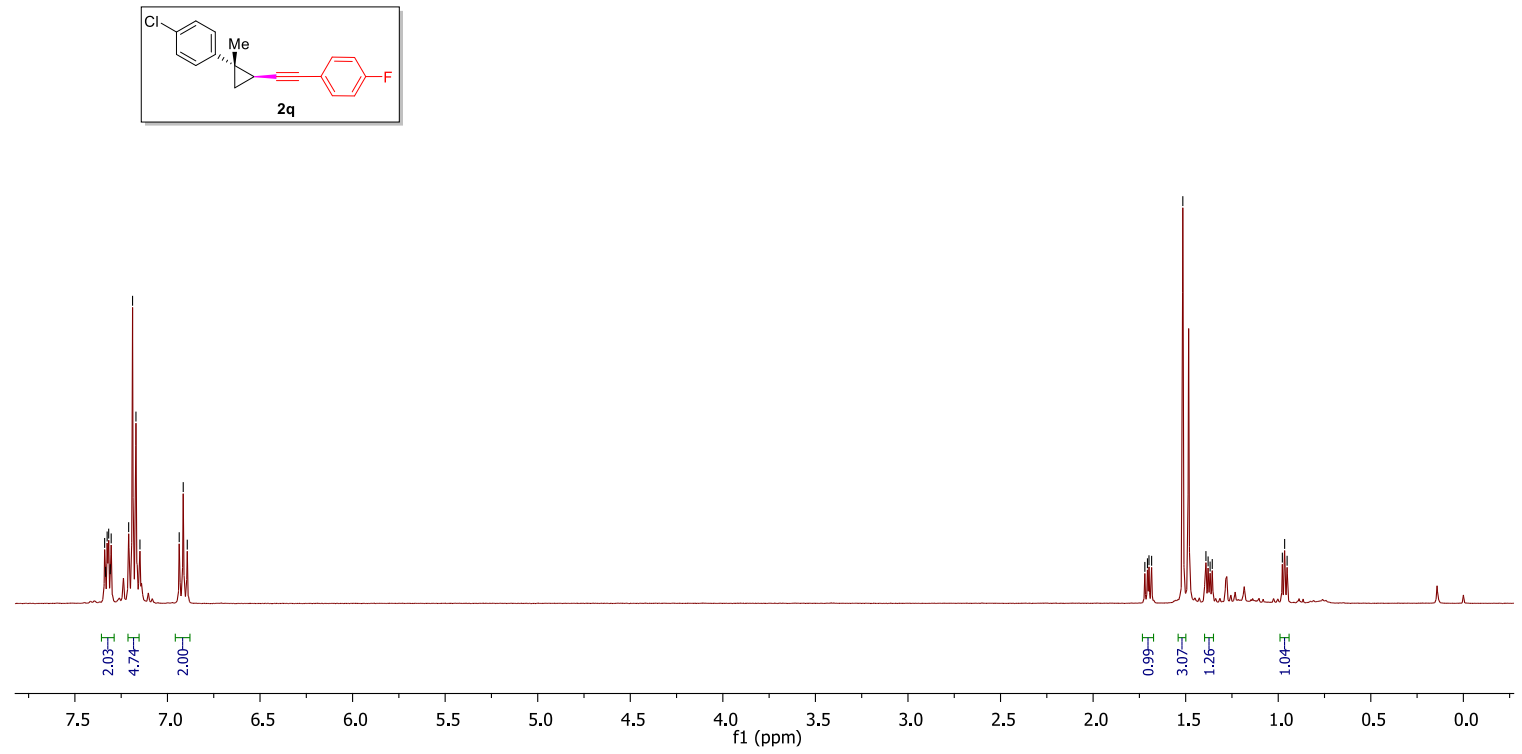

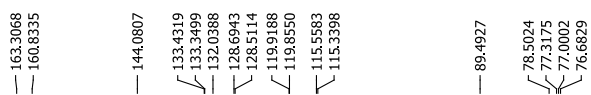

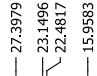
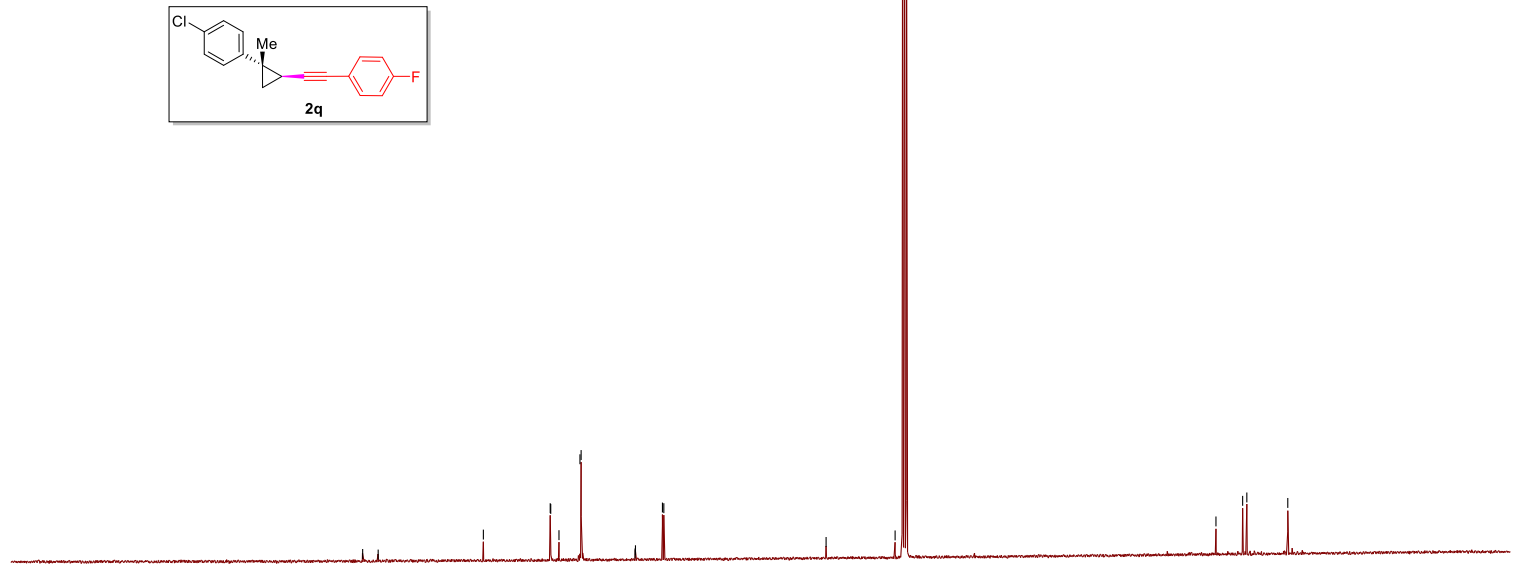
Supporting Information

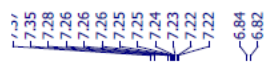

$\stackrel{\vec{m}}{\tilde{i}}$

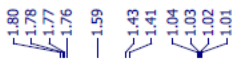
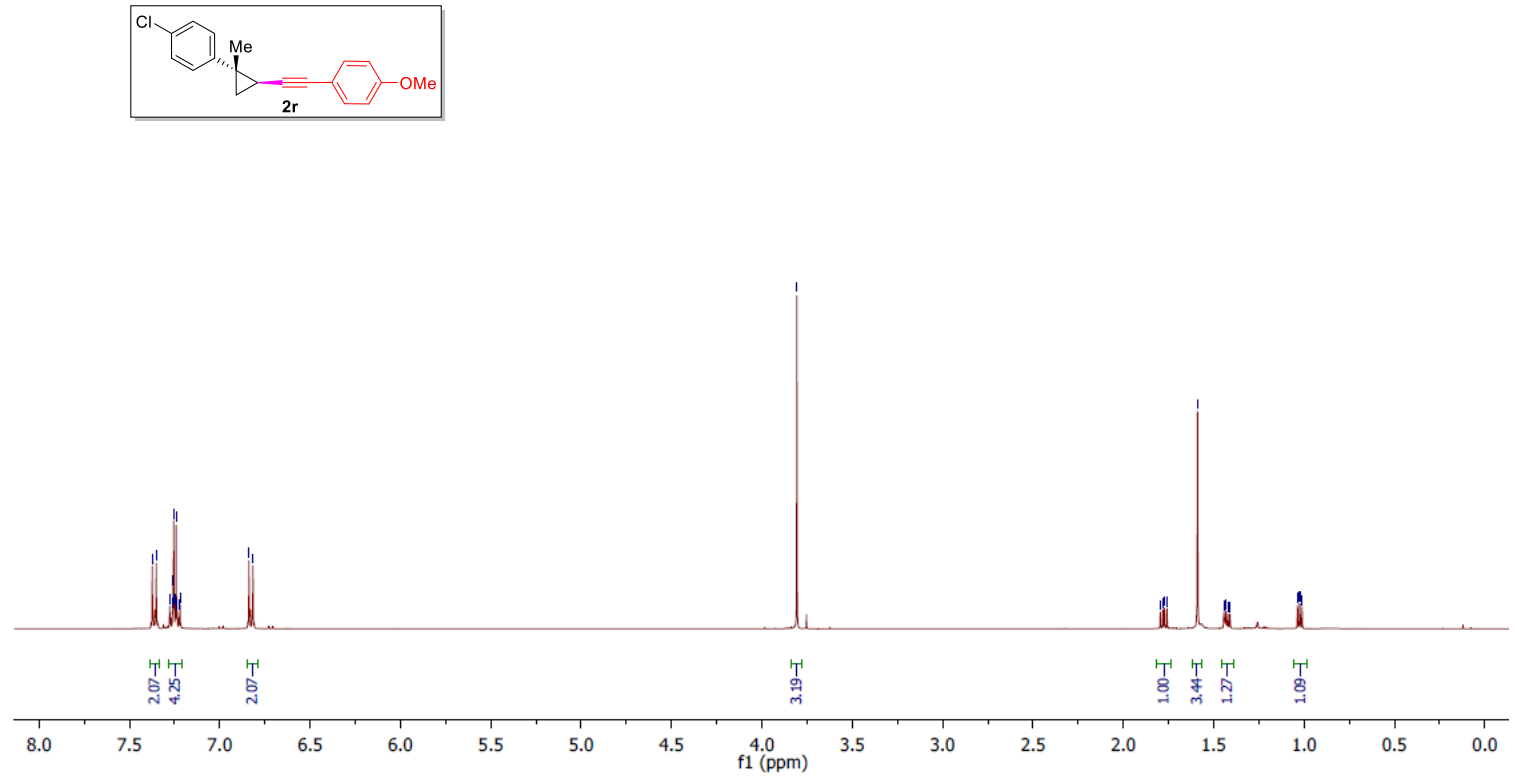

dly-X01703p

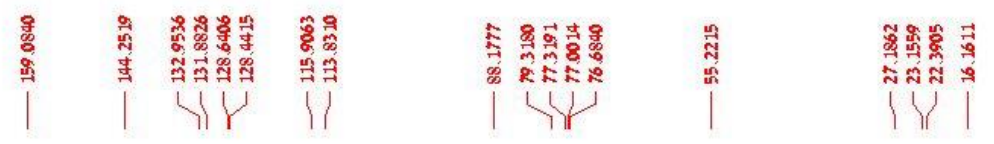

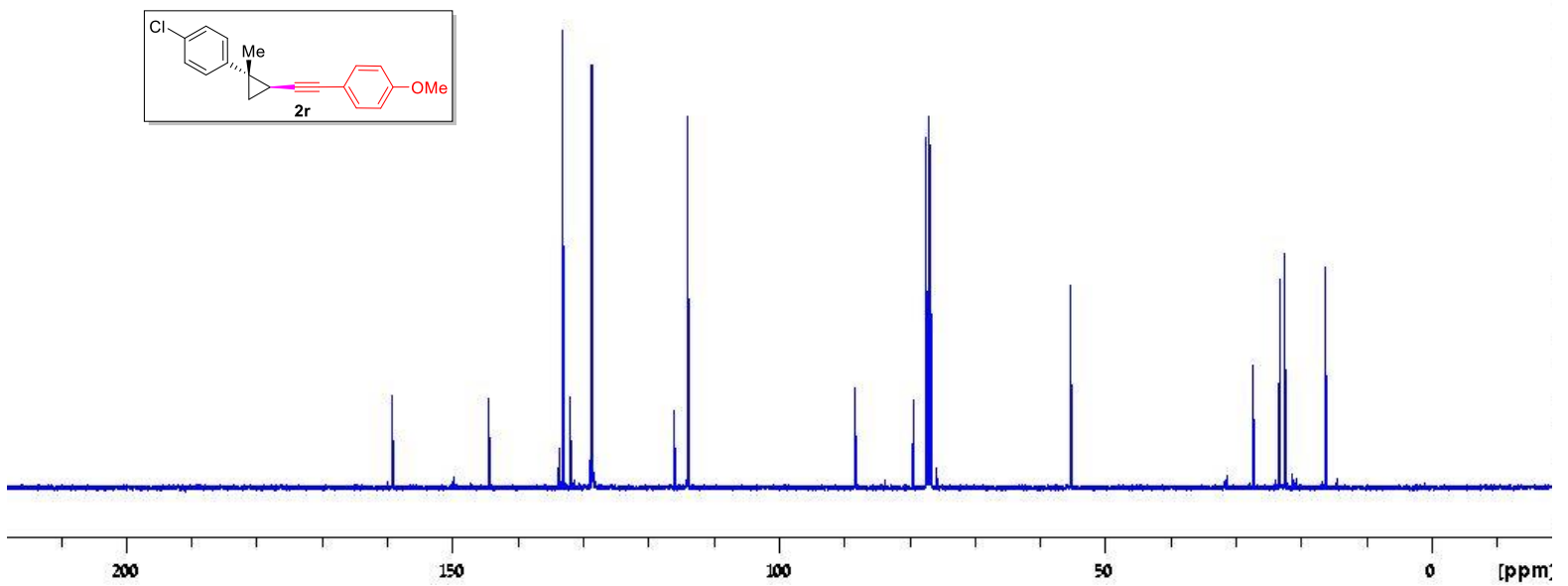


Supporting Information

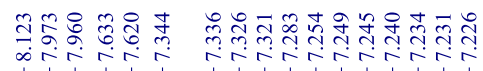

每
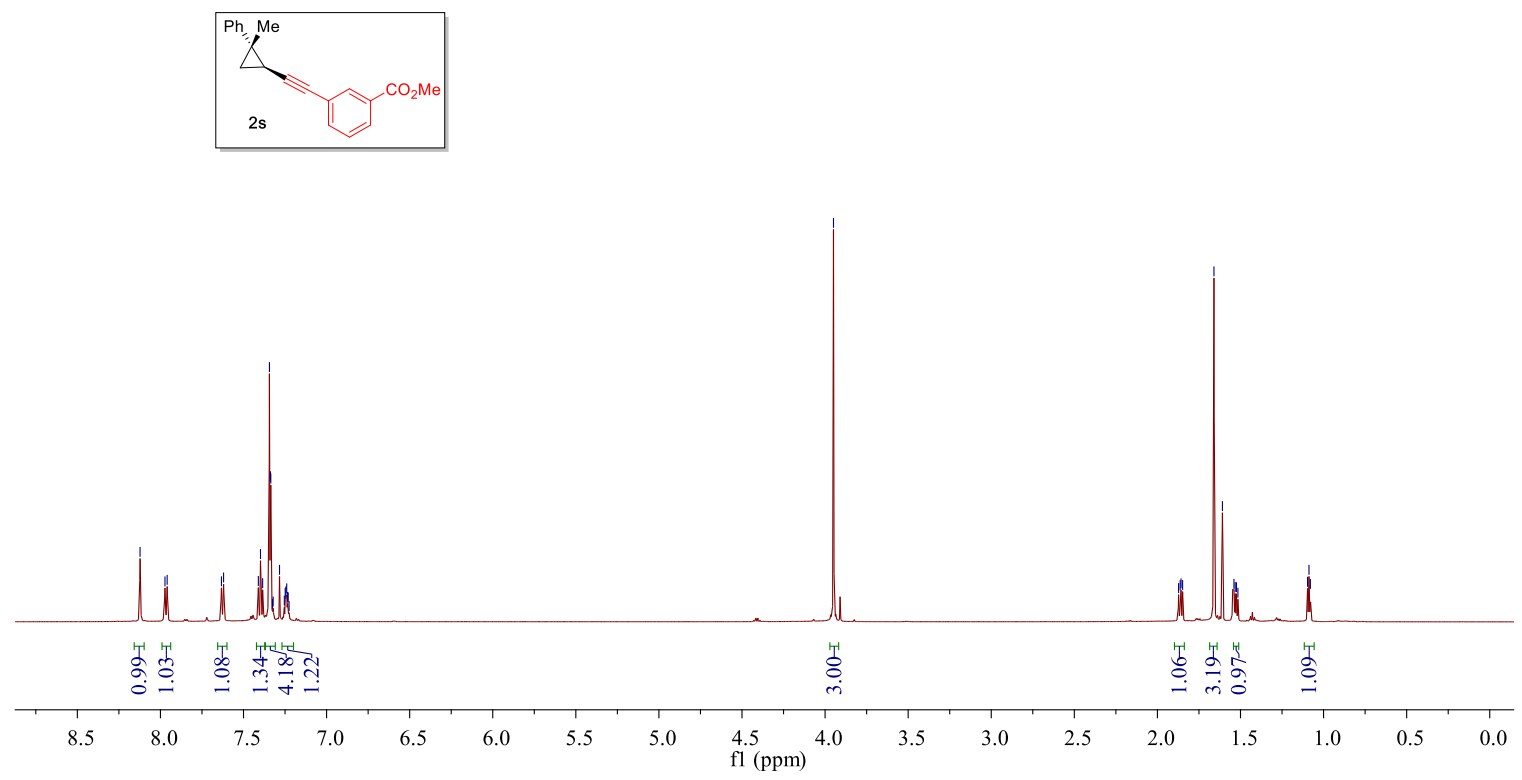

\begin{tabular}{|c|c|c|c|c|}
\hline 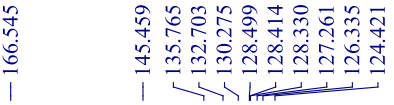 & 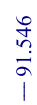 & 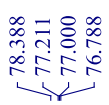 & 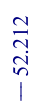 & 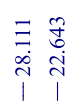 \\
\hline
\end{tabular}
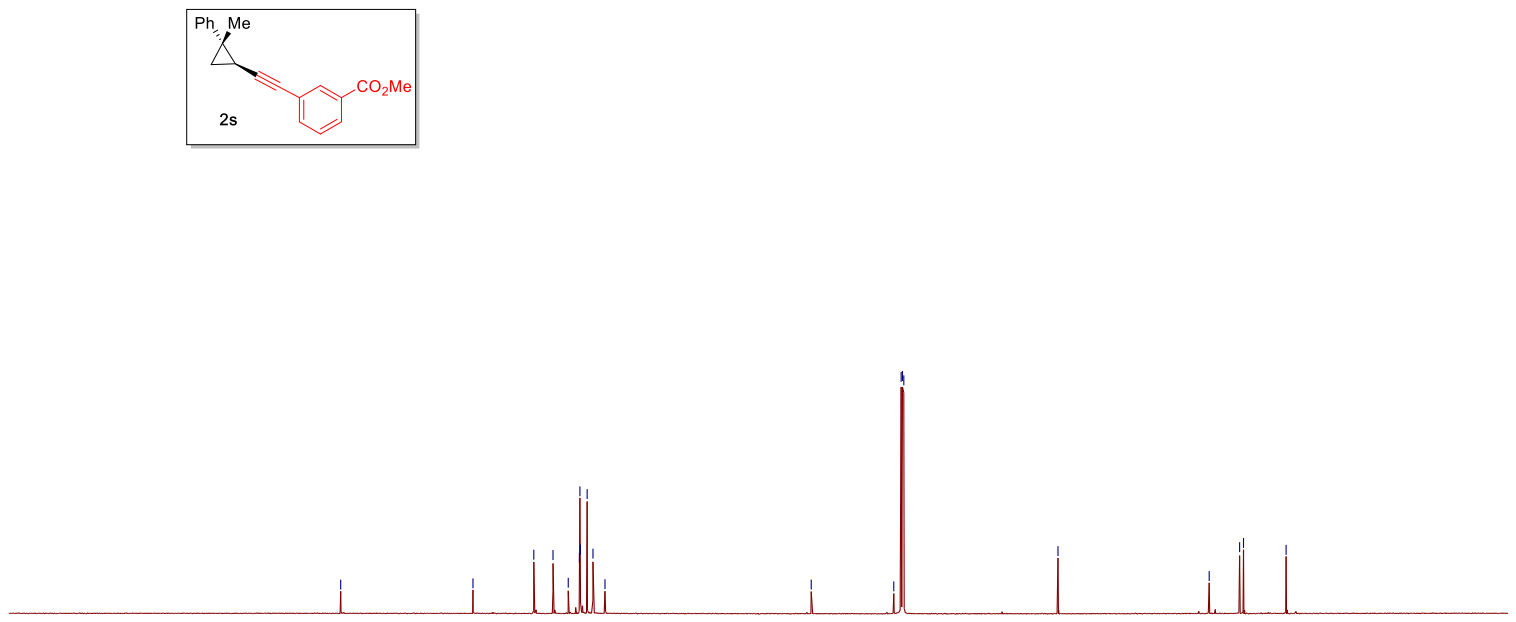

$\begin{array}{llllllllllllllllllllllllllll}210 & 200 & 190 & 180 & 170 & 160 & 150 & 140 & 130 & 120 & 110 & 100 & 90 & 80 & 70 & 60 & 50 & 40 & 30 & 20 & 10 & 0 & -10\end{array}$ 
Supporting Information

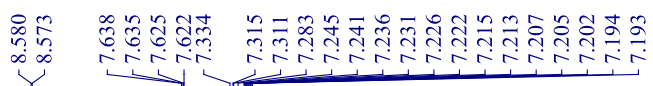

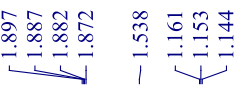
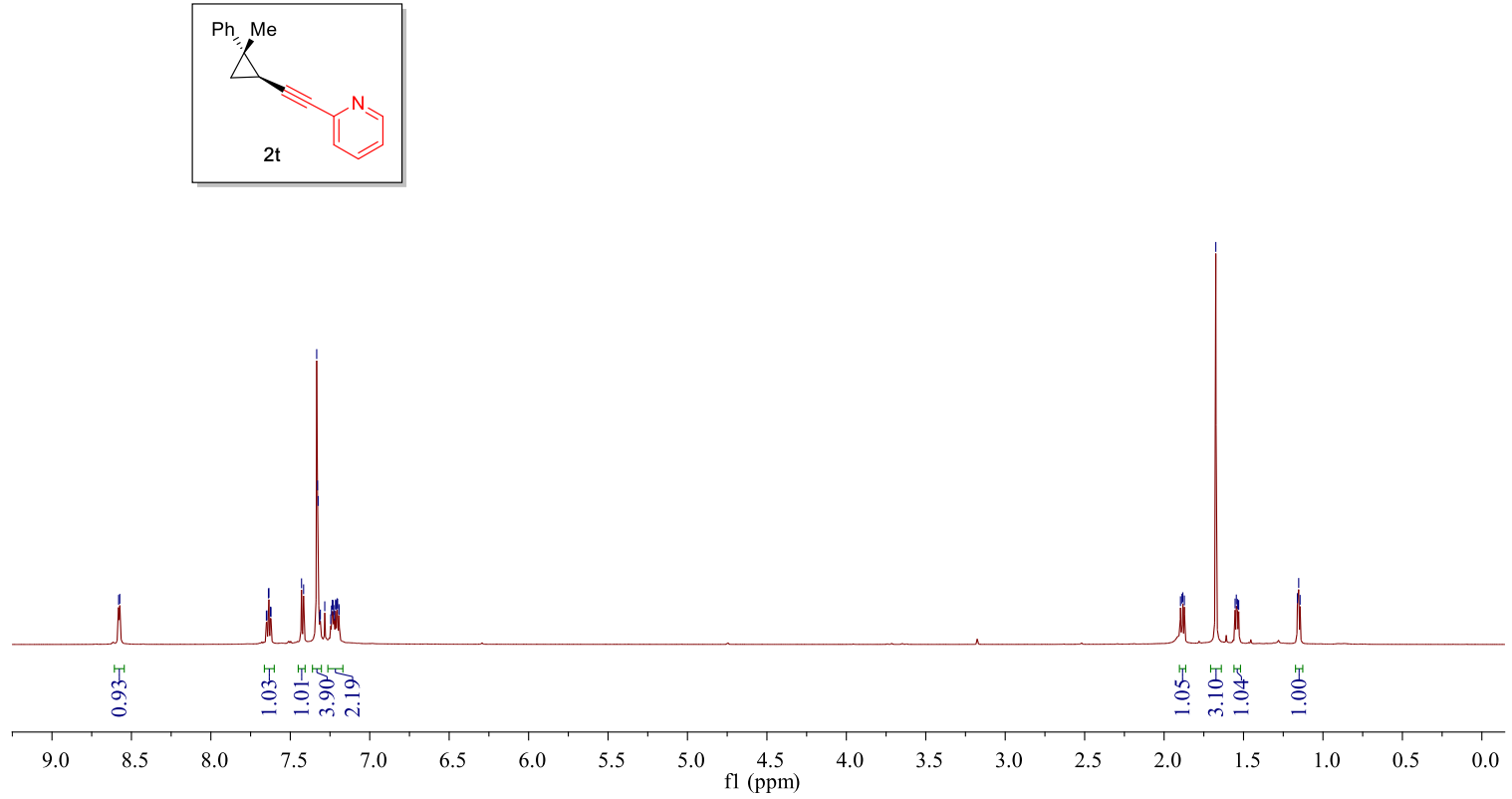

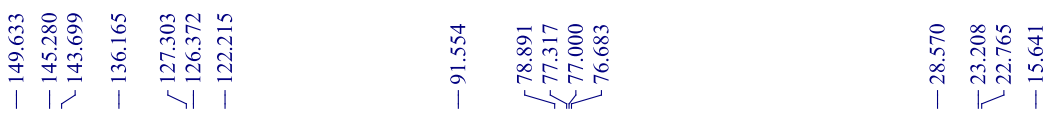
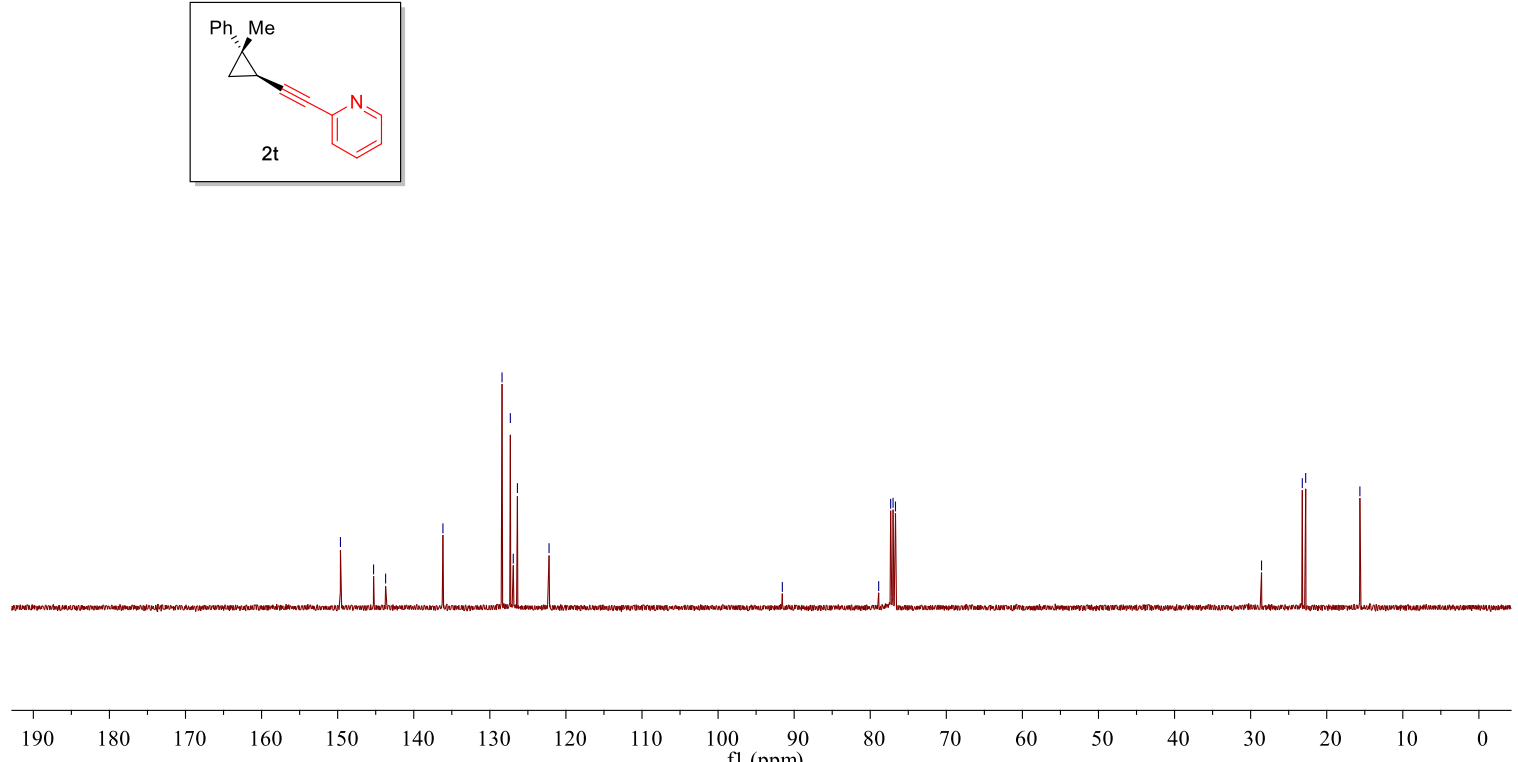
Supporting Information
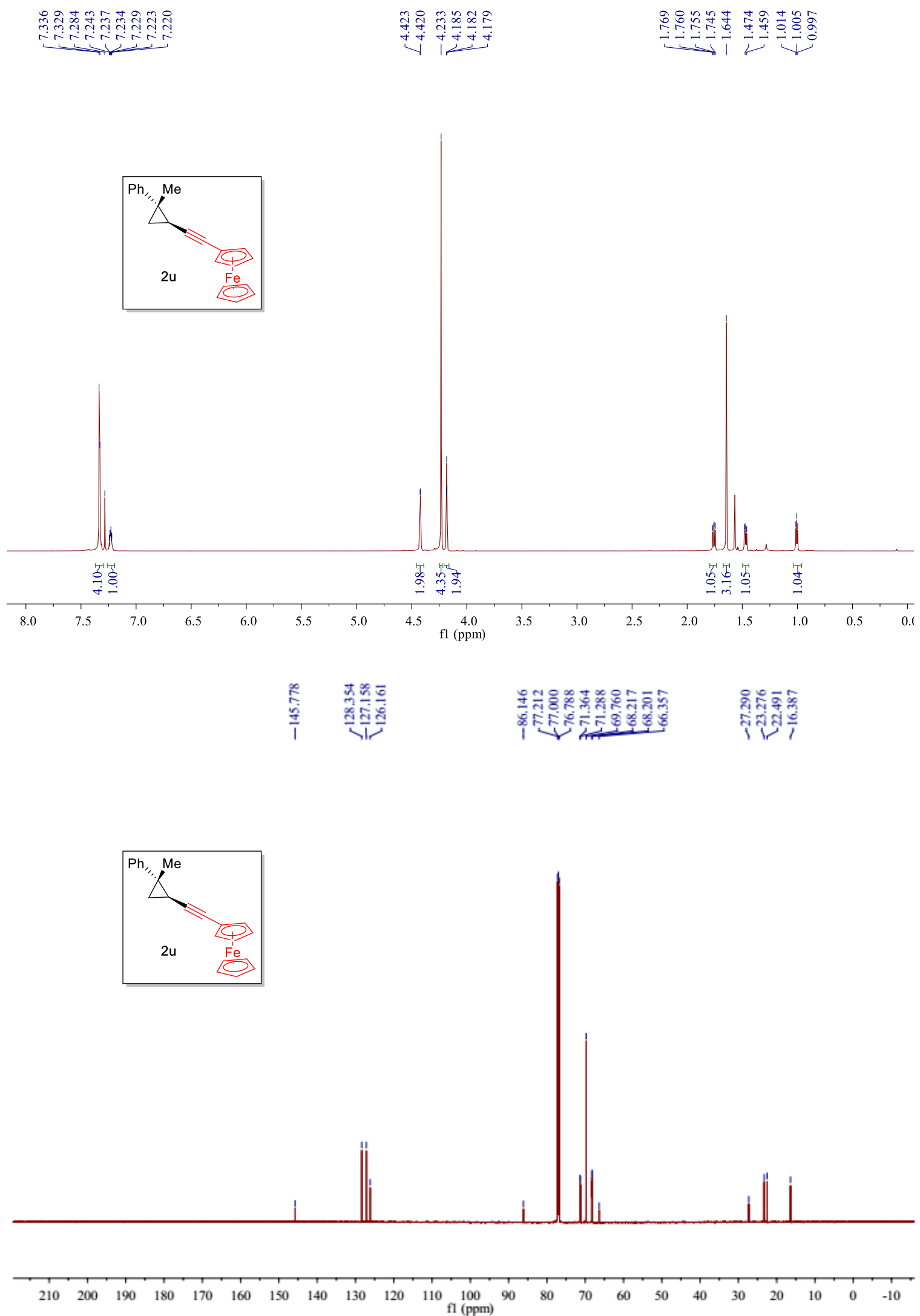
Supporting Information
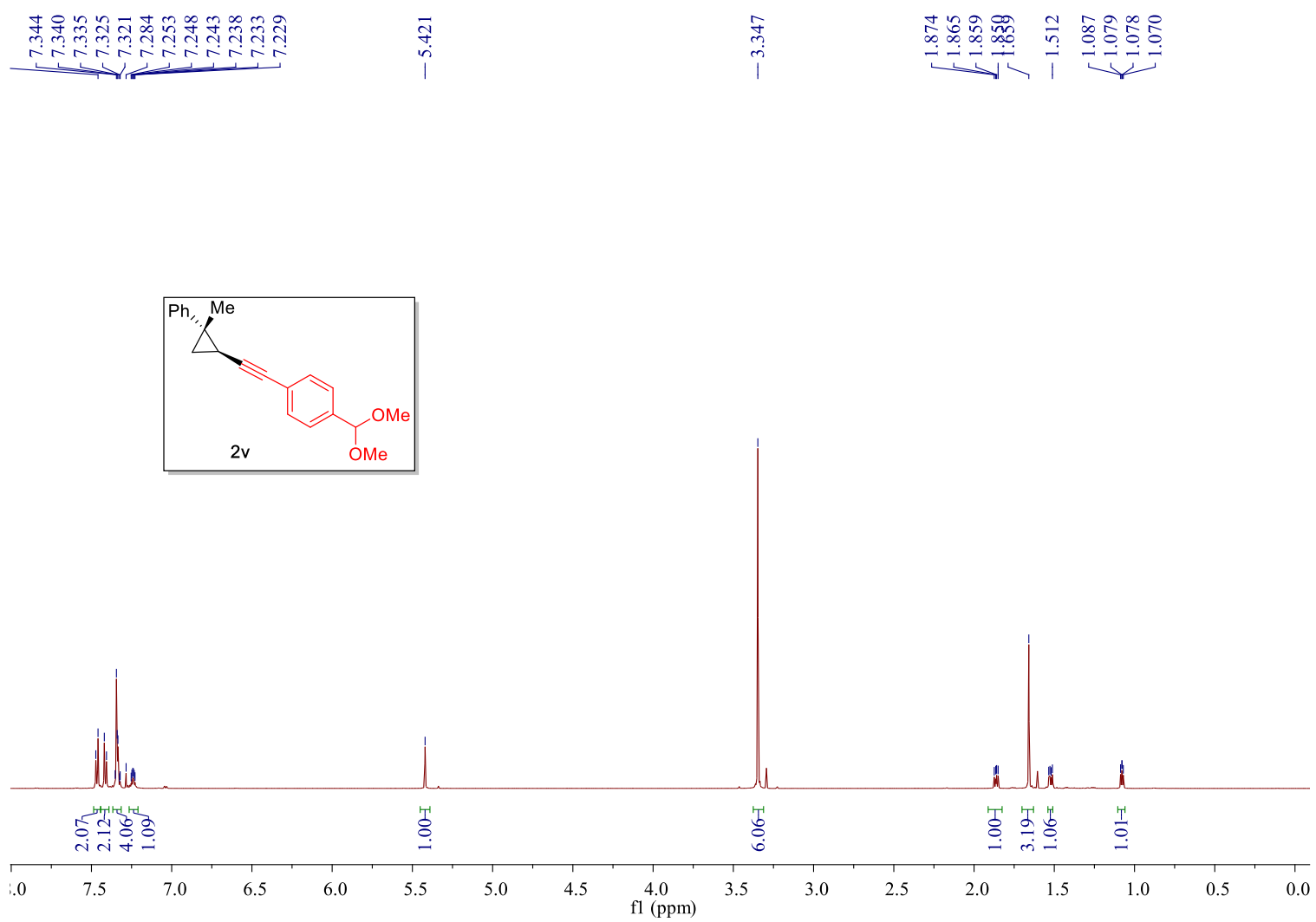

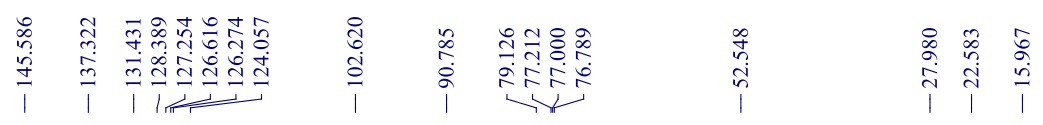
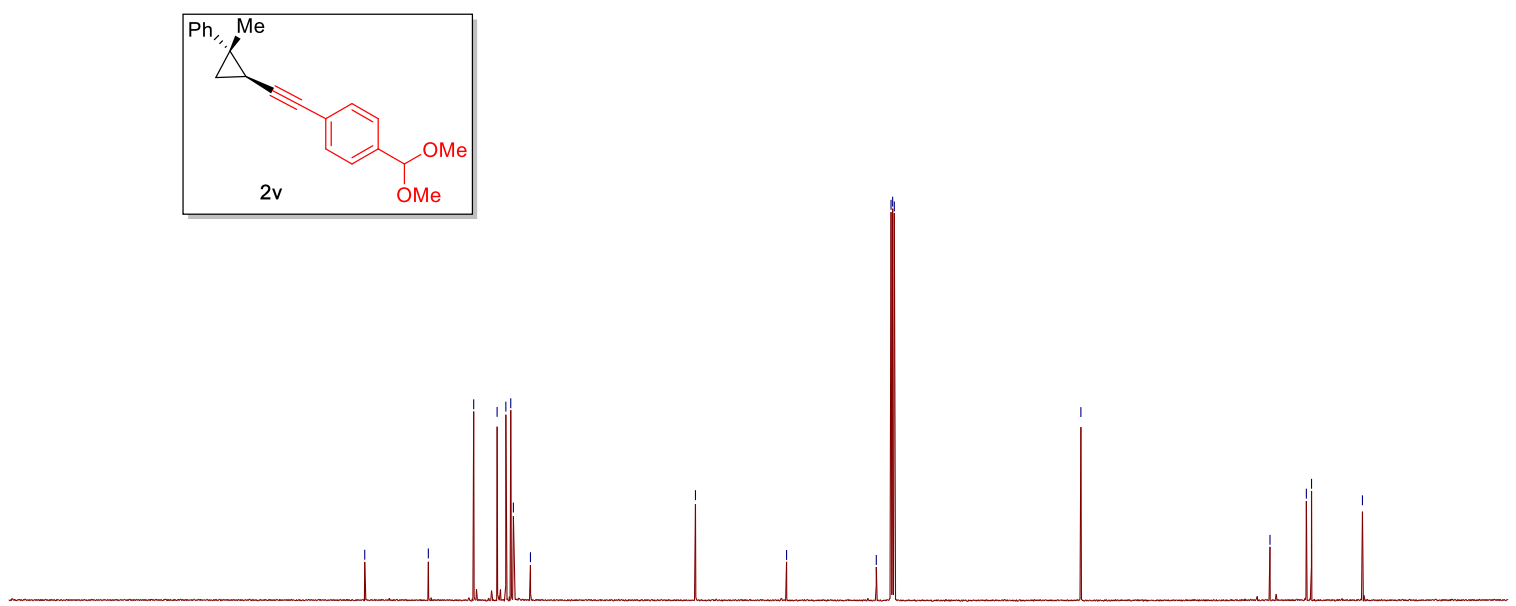

19

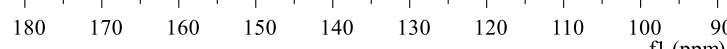

fl (ppm)

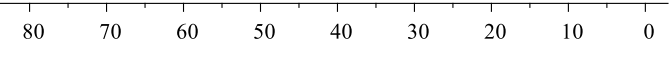



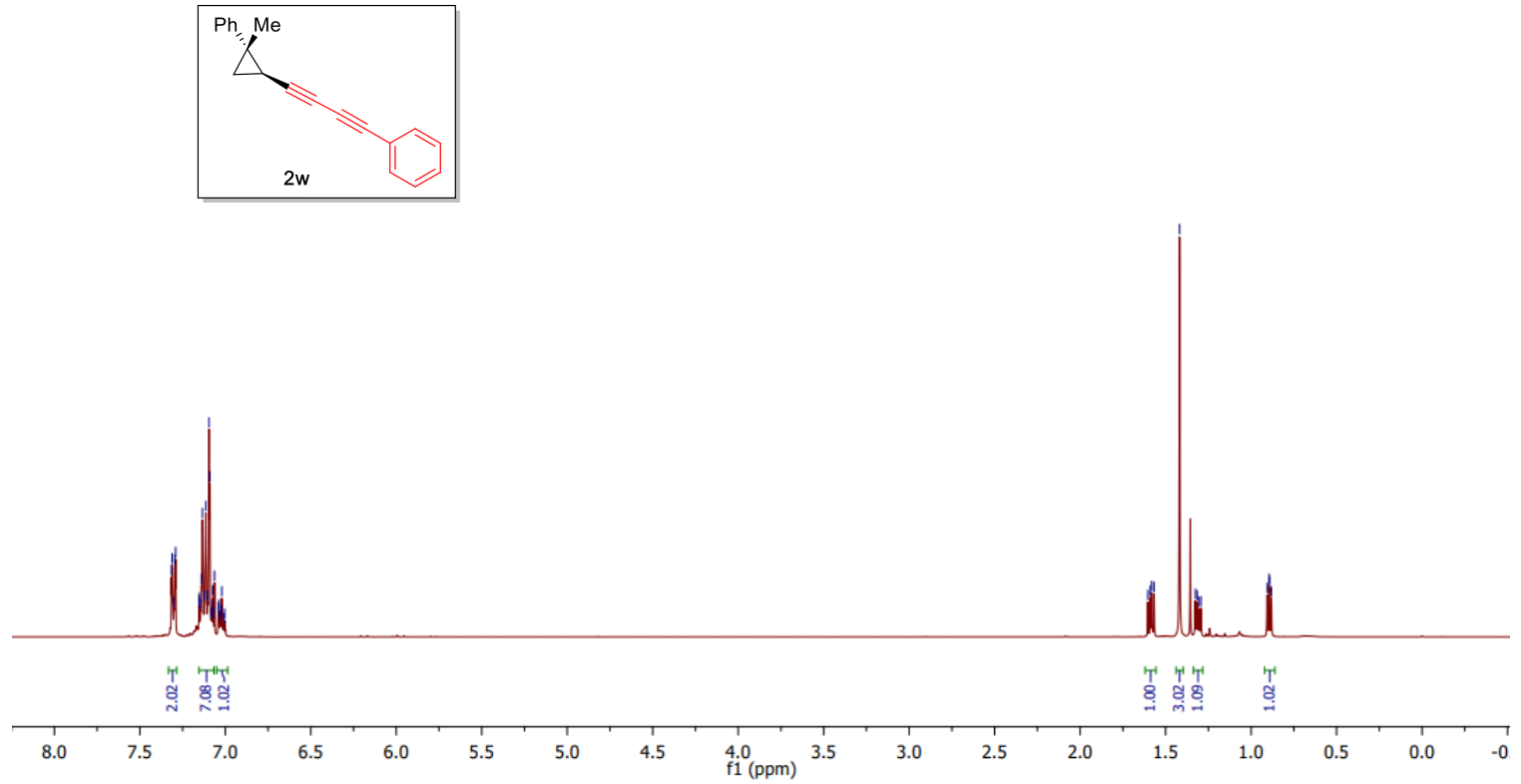

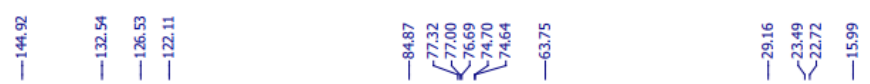
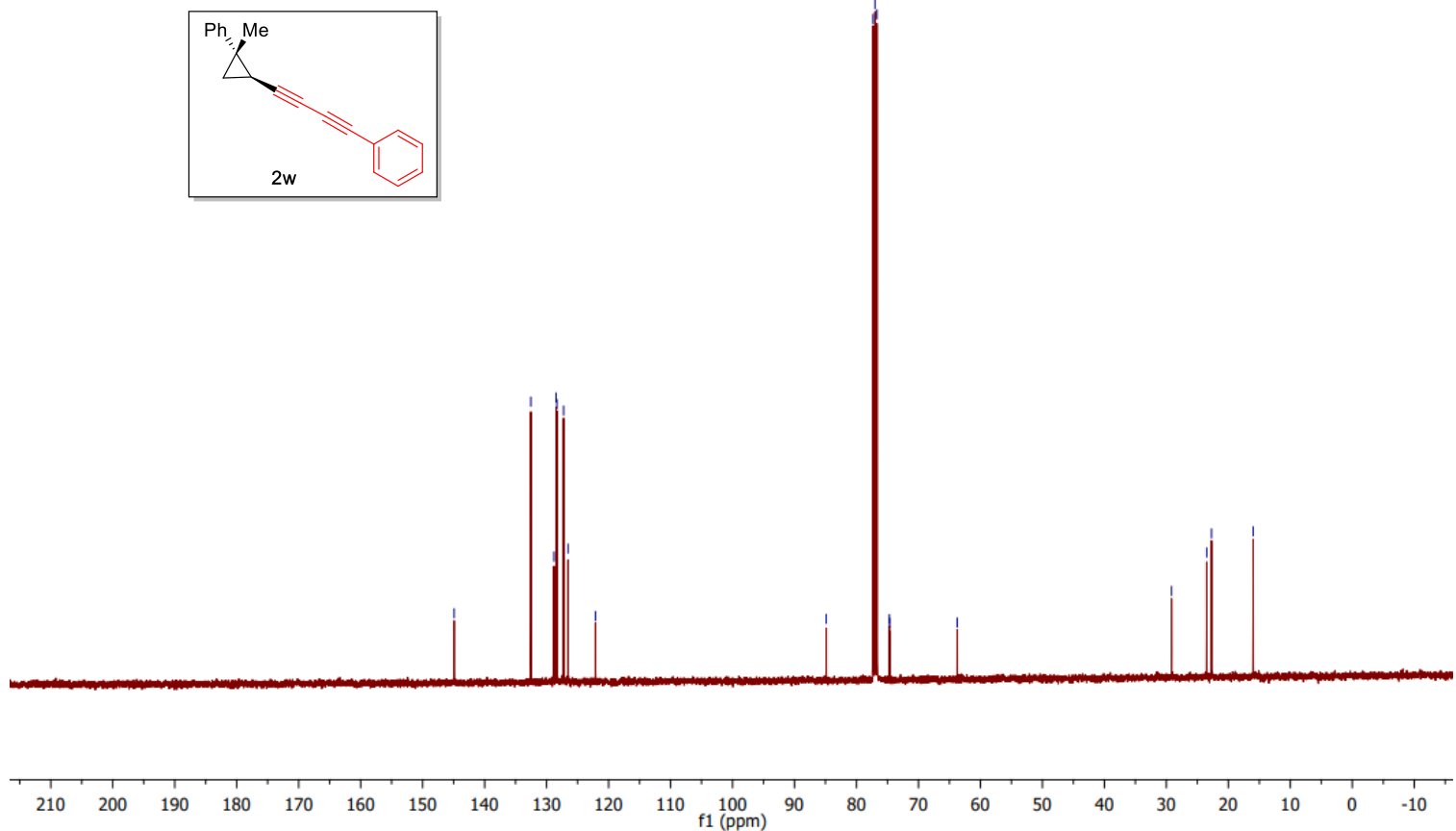
Supporting Information

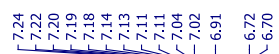

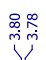

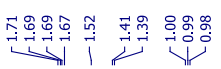
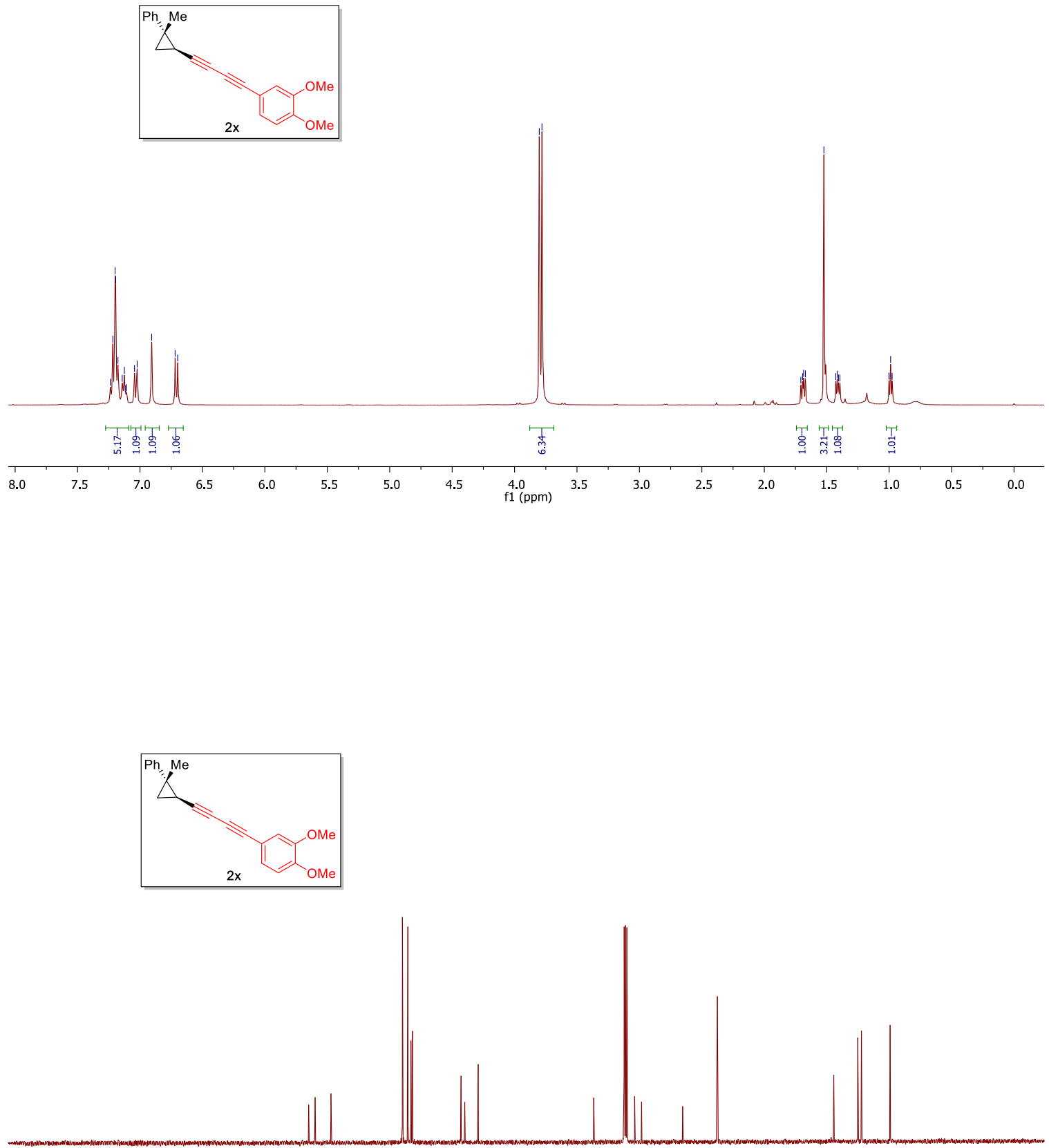

$\begin{array}{llllllllllllllllllllllllllllll}210 & 200 & 190 & 180 & 170 & 160 & 150 & 140 & 130 & 120 & 110 & \begin{array}{c}100 \\ \mathrm{f} 1(\mathrm{ppm})\end{array} & 90 & 80 & 70 & 60 & 50 & 40 & 30 & 20 & 10 & 0 & -10\end{array}$ 
Supporting Information

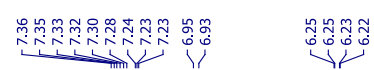

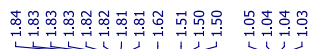
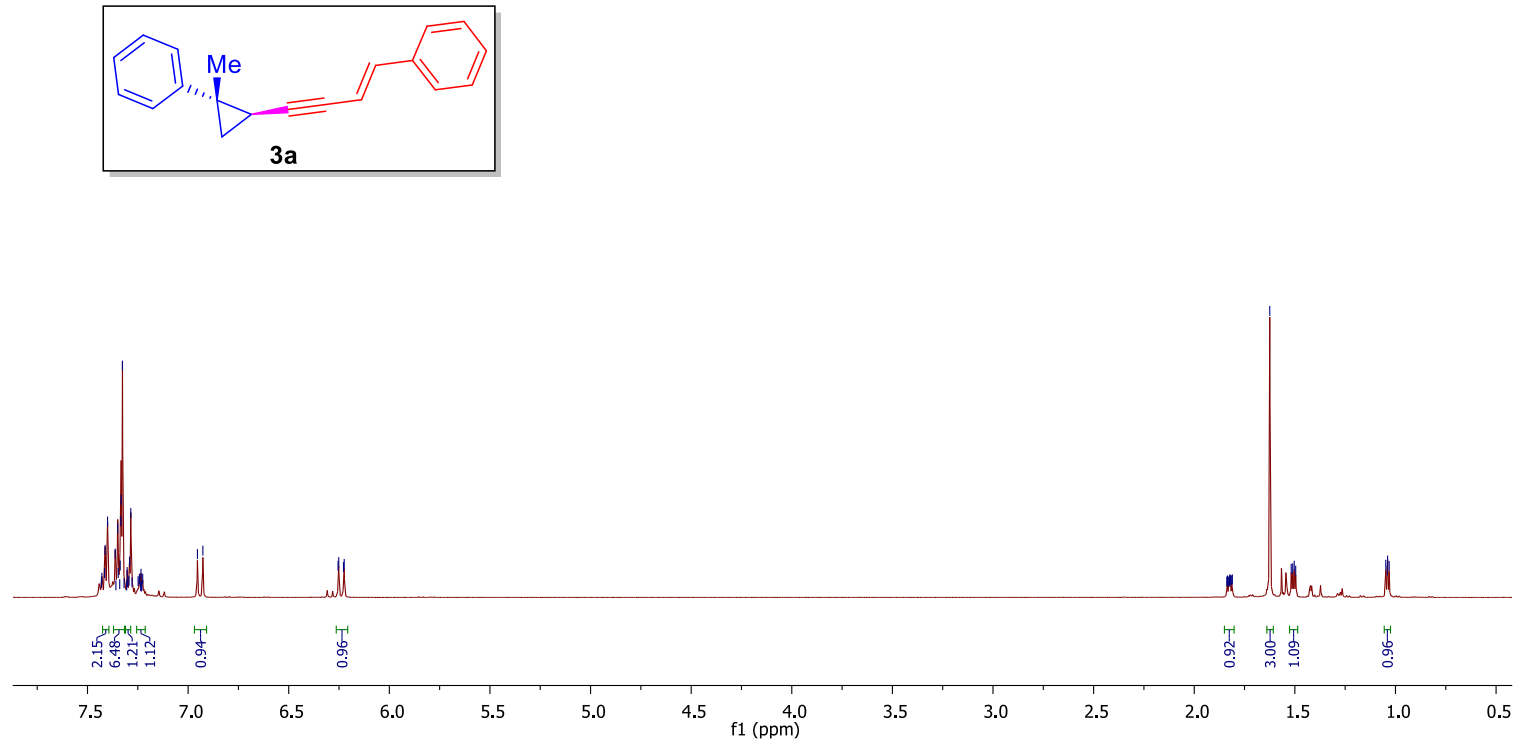

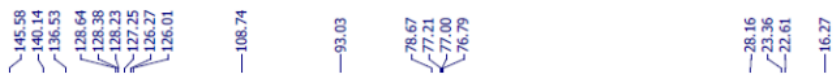
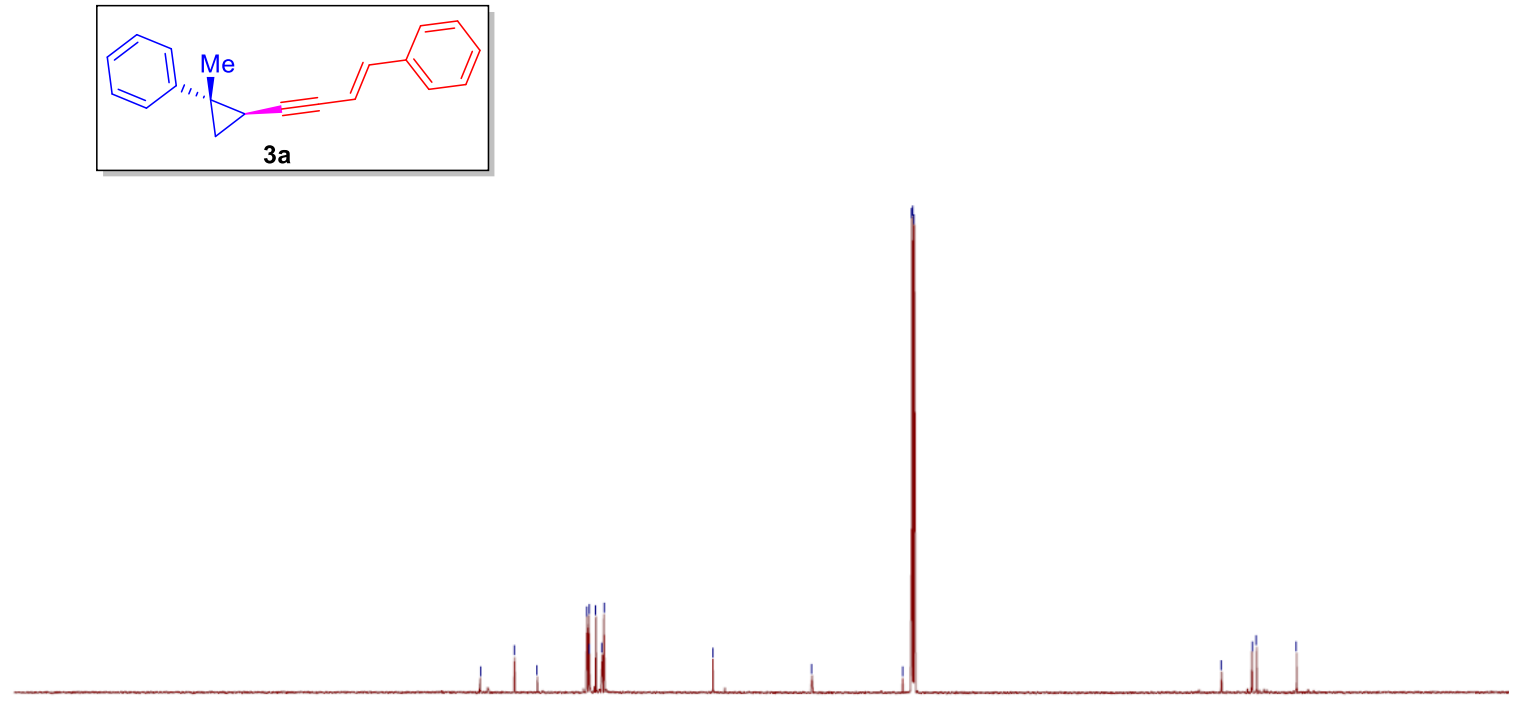

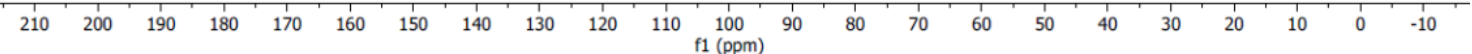


Supporting Information
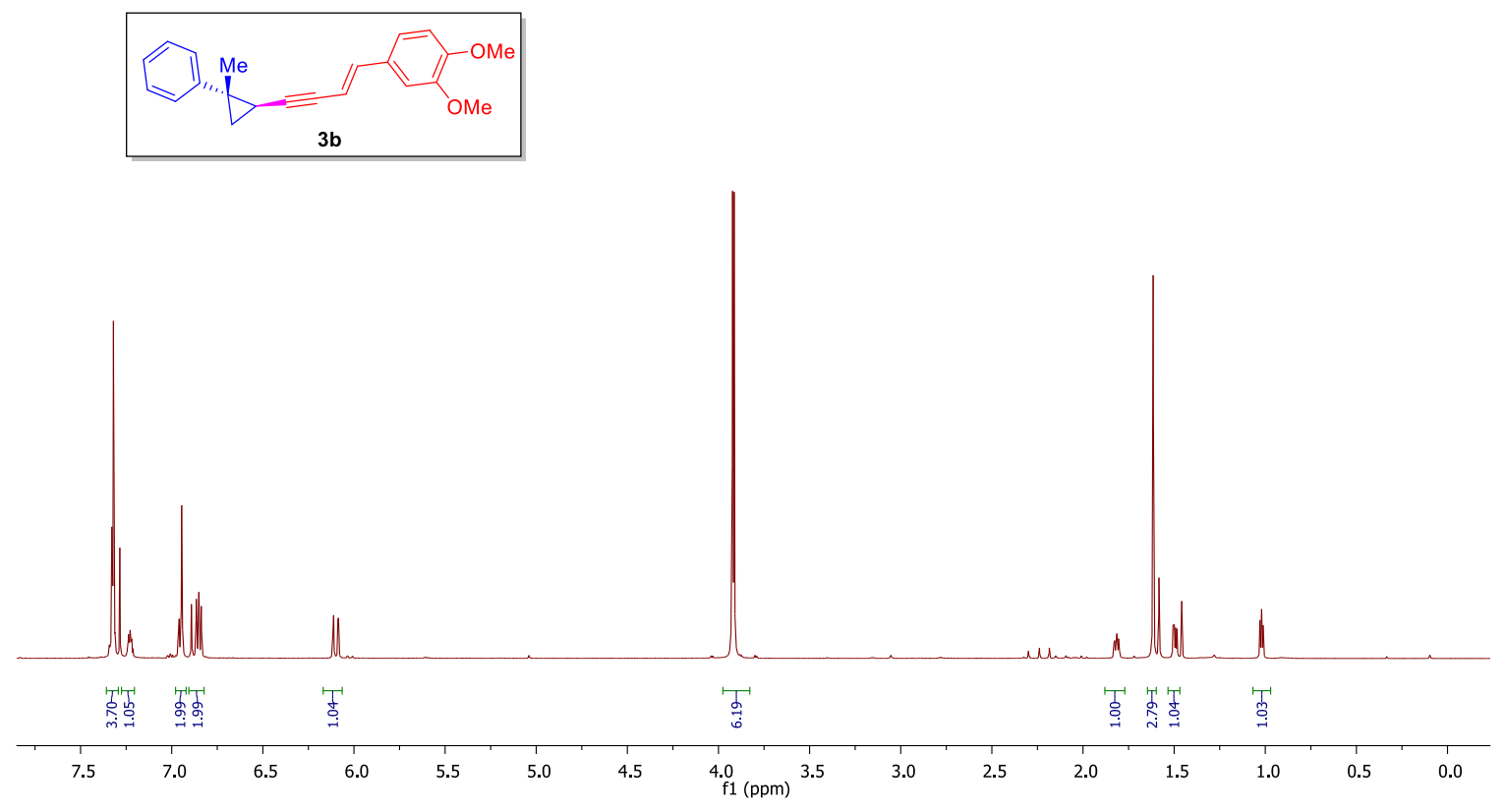

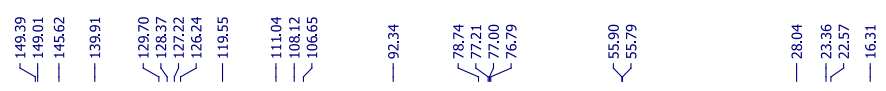
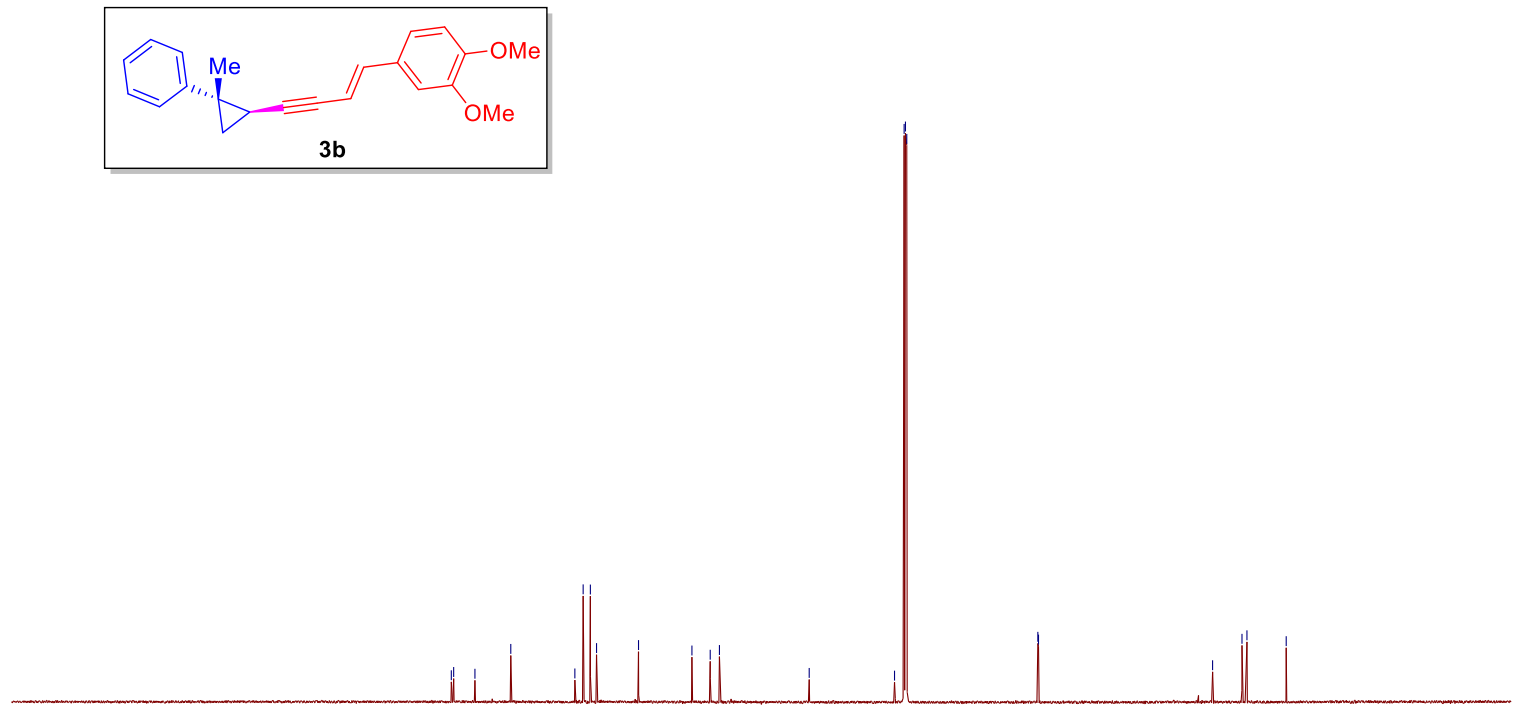
Supporting Information
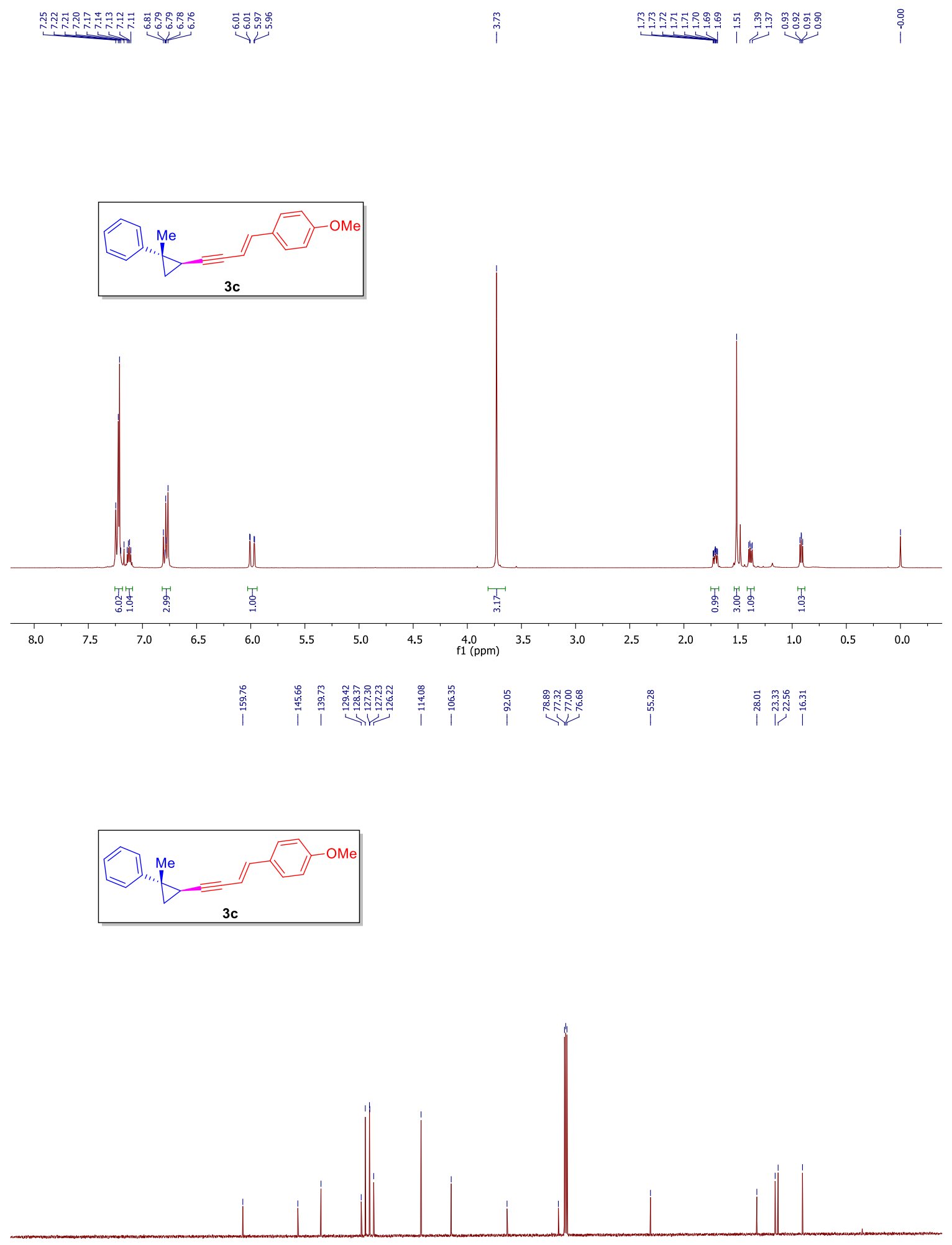
Supporting Information

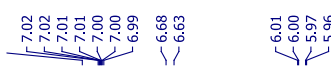

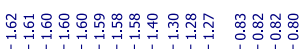
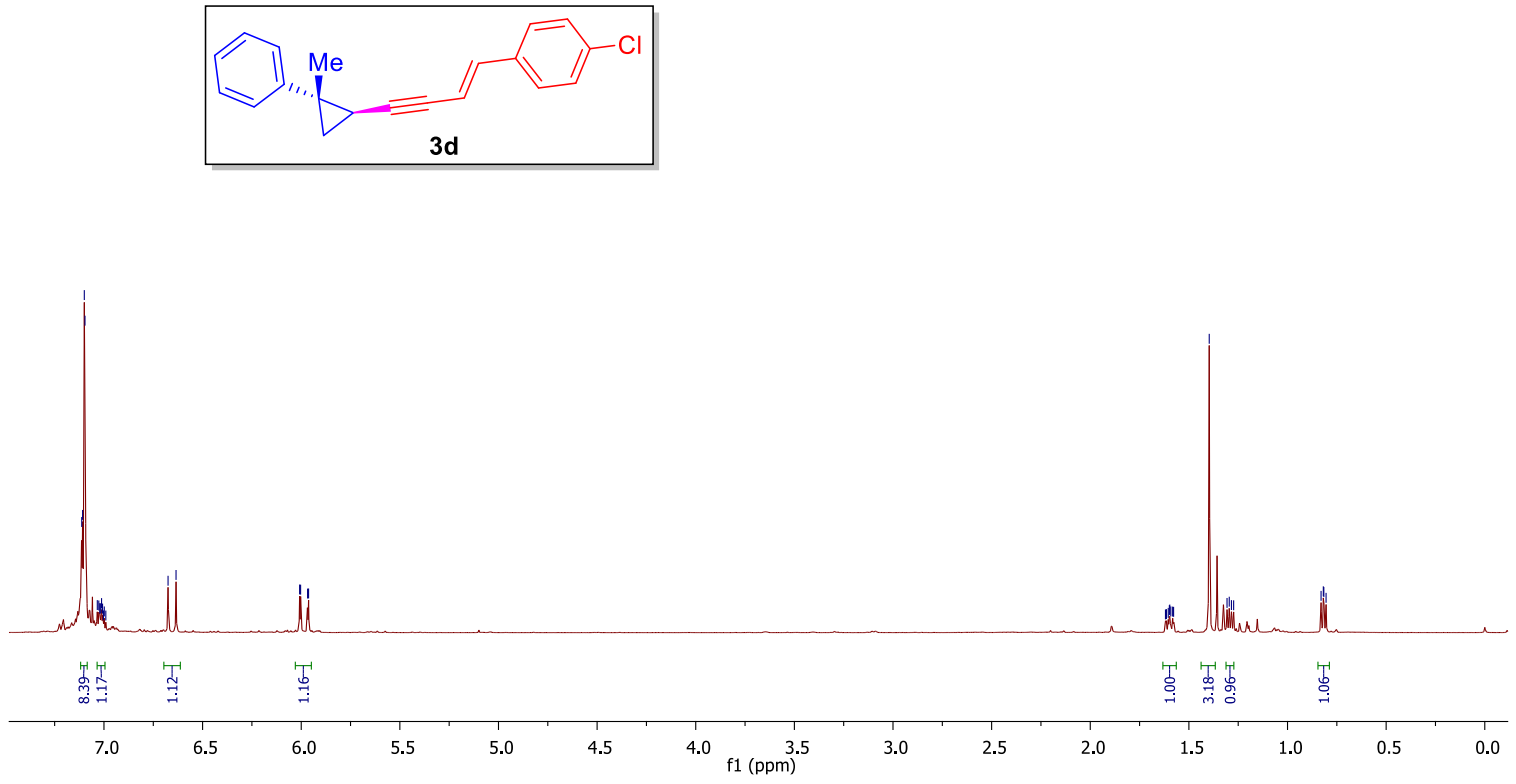

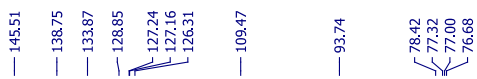

i
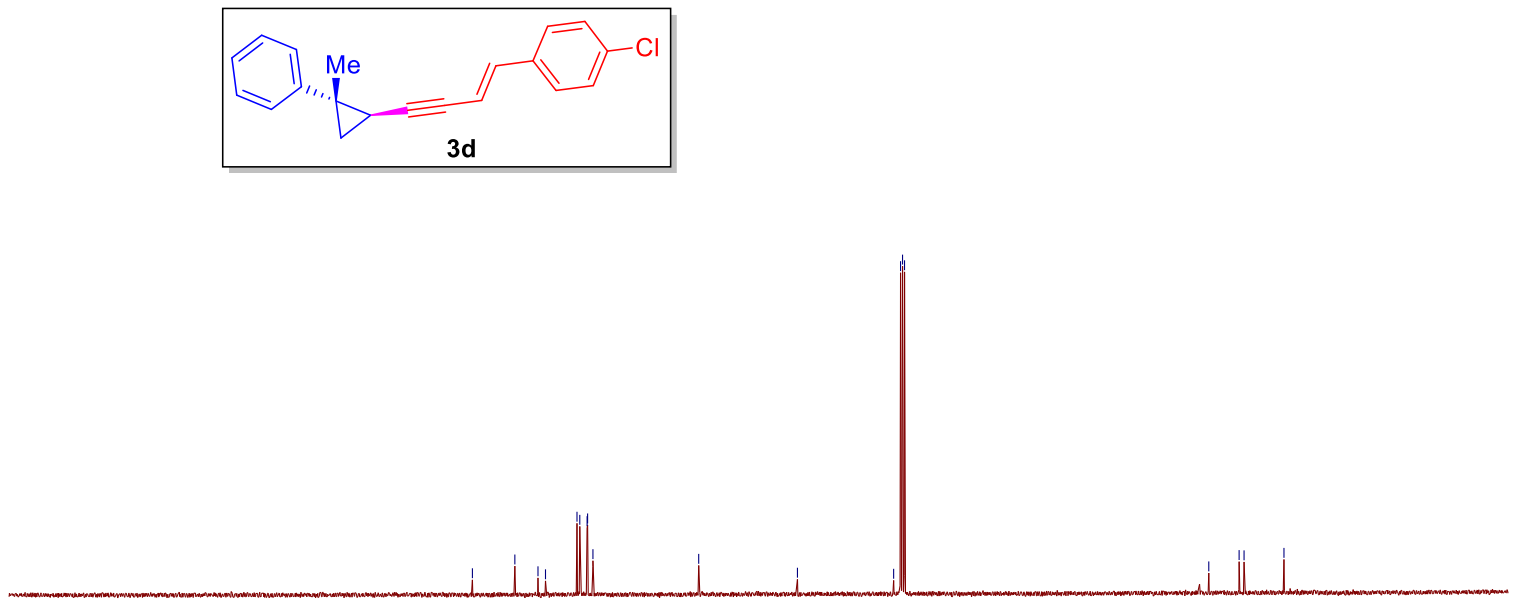
Supporting Information

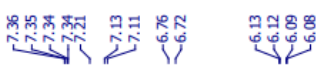

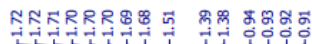
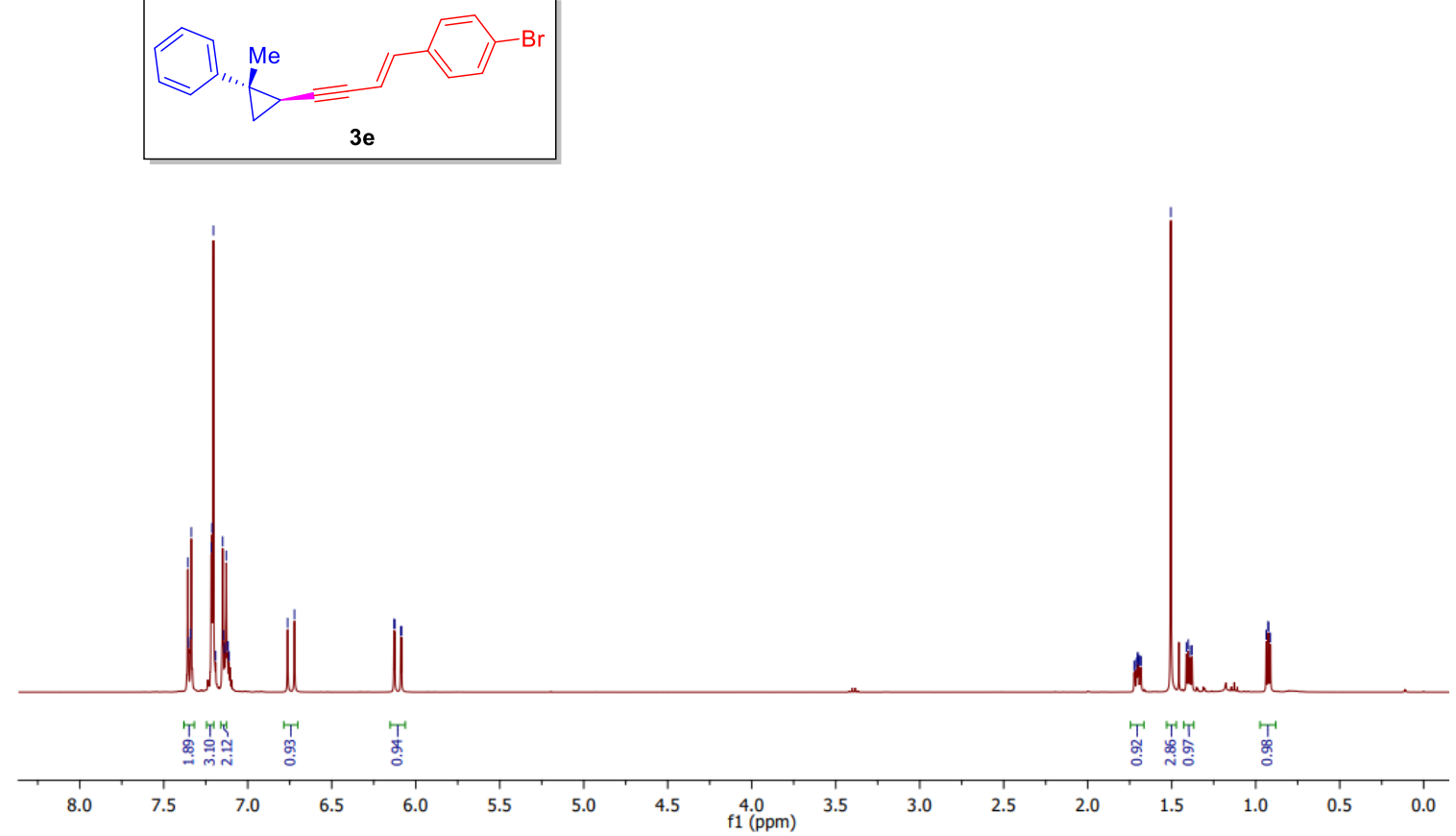

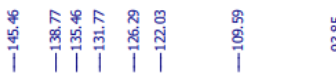

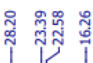
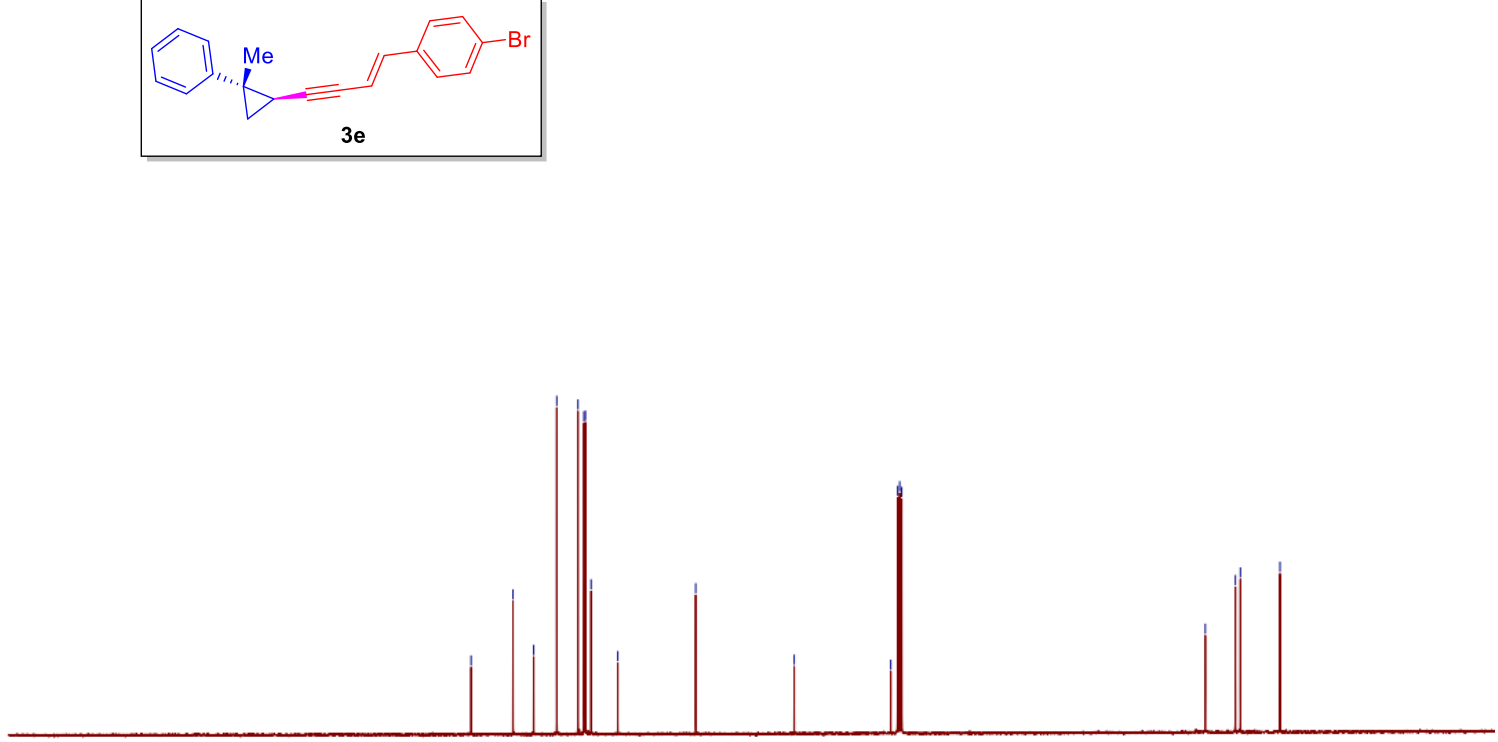
Supporting Information

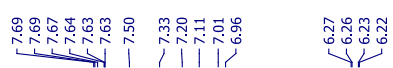

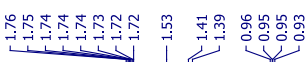
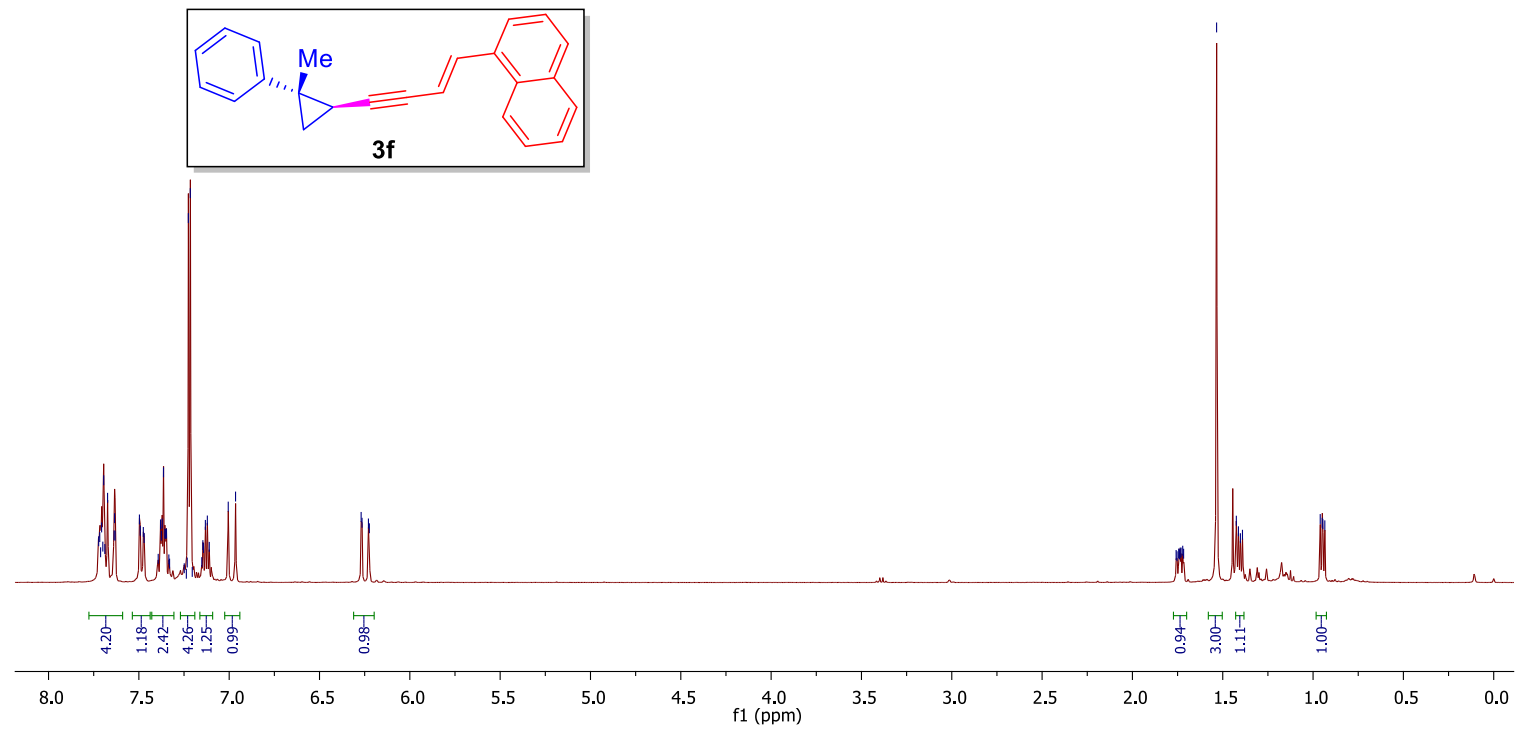

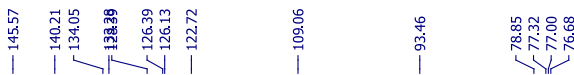

|
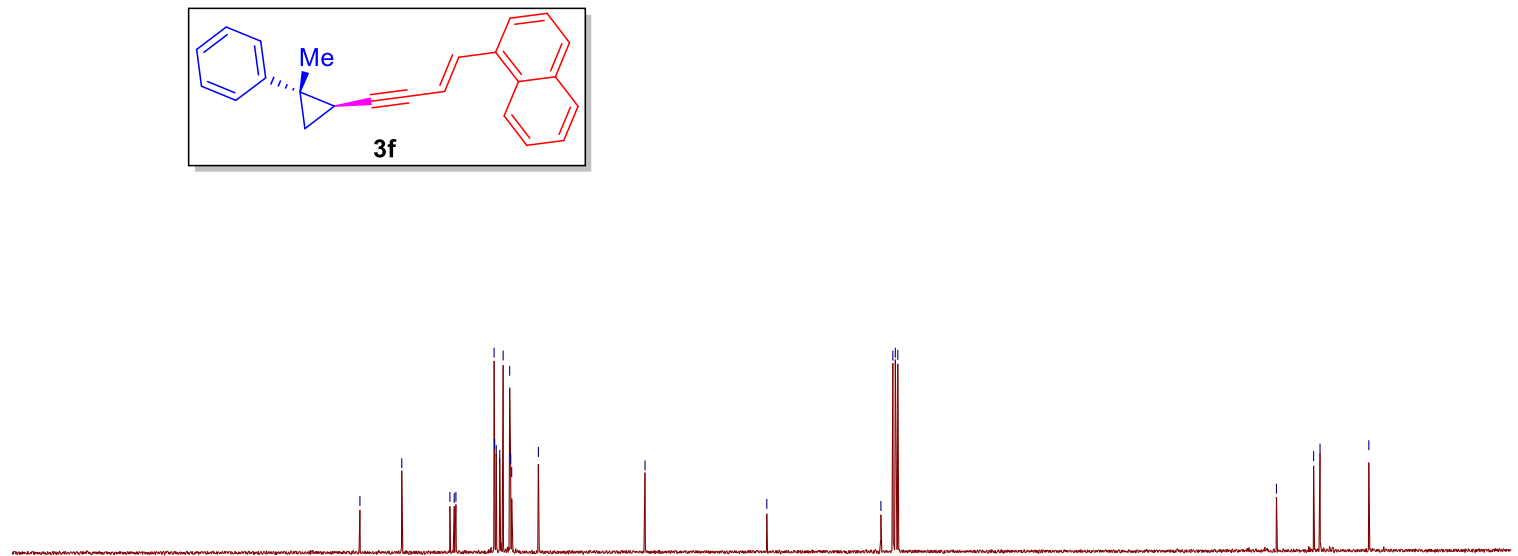

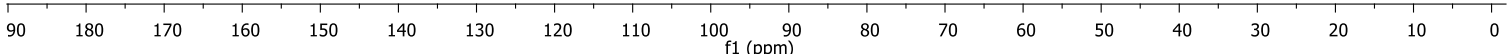


Supporting Information

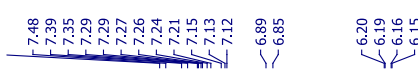

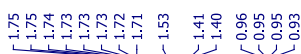
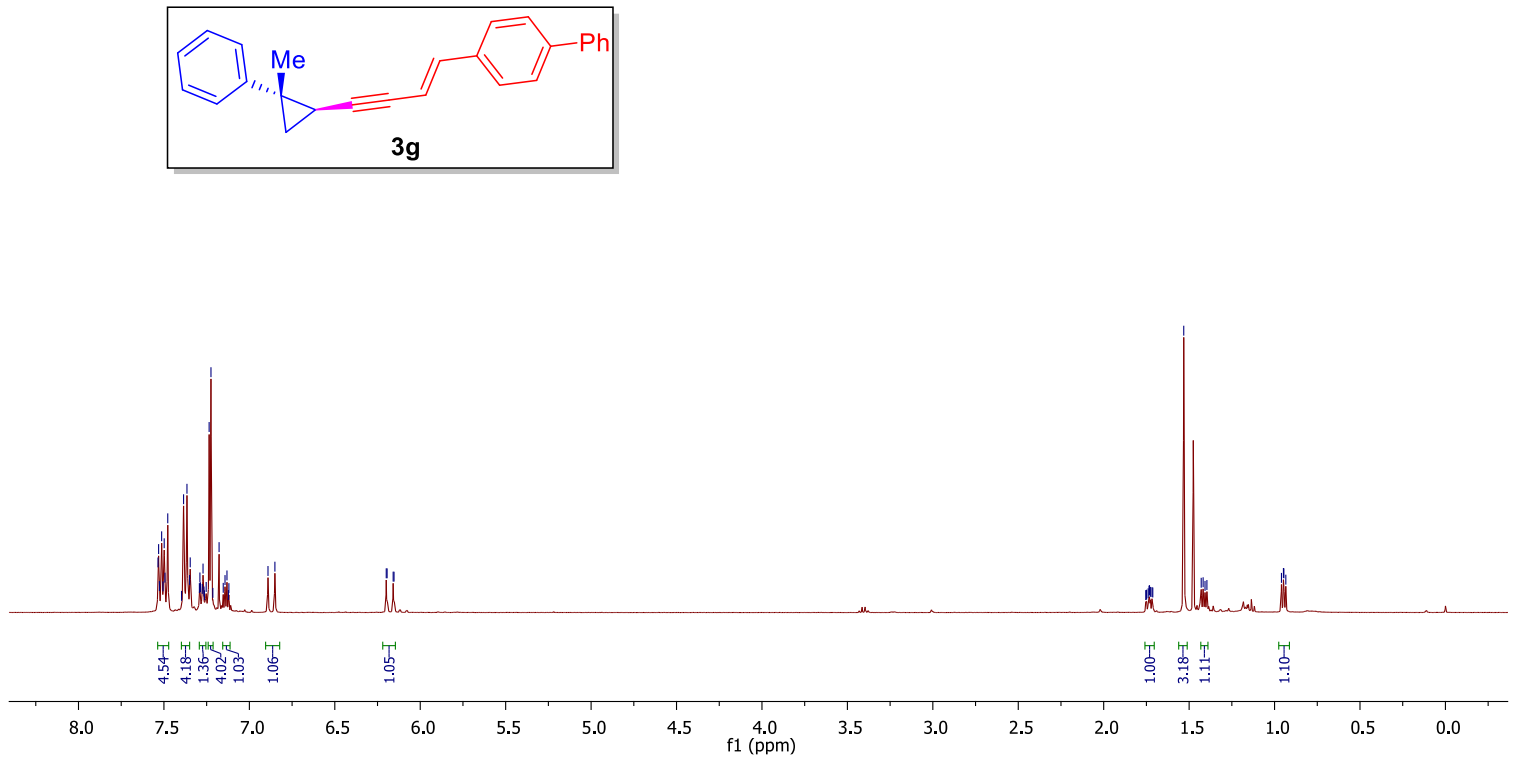

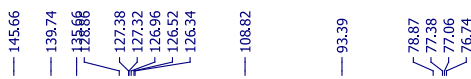

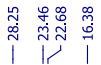
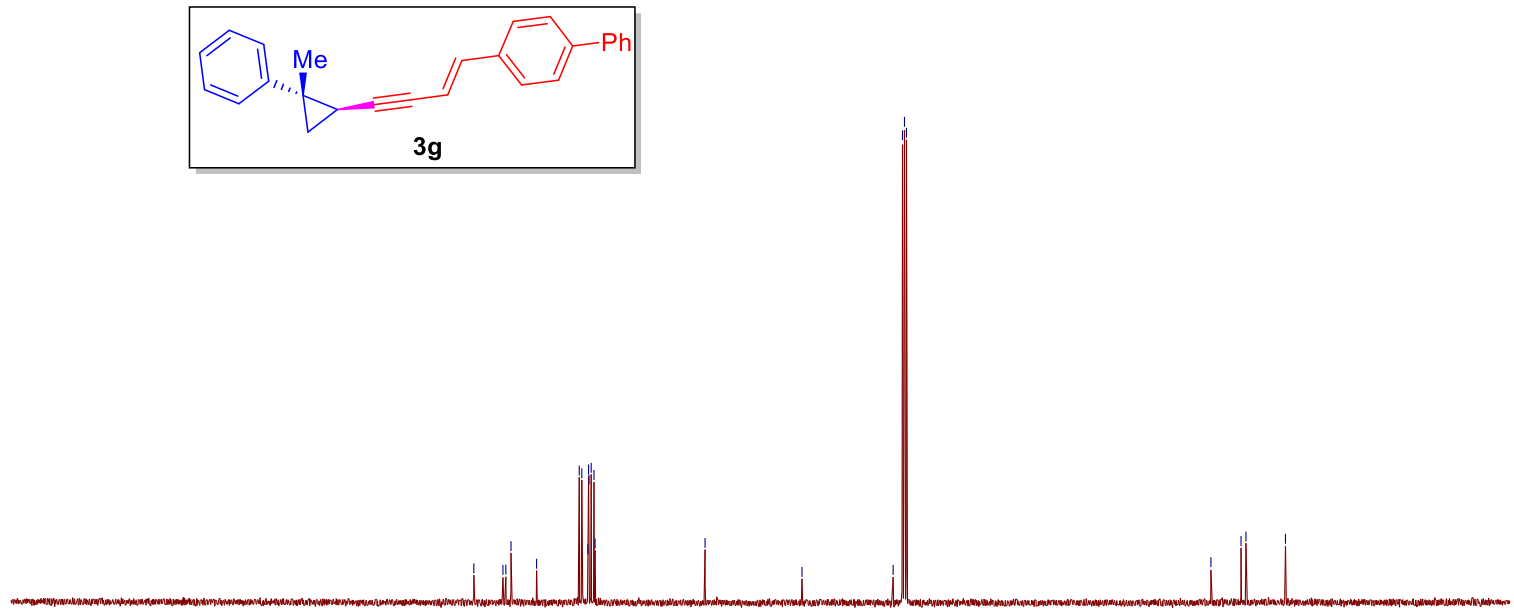
Supporting Information

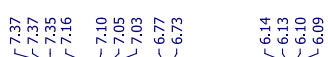

虫
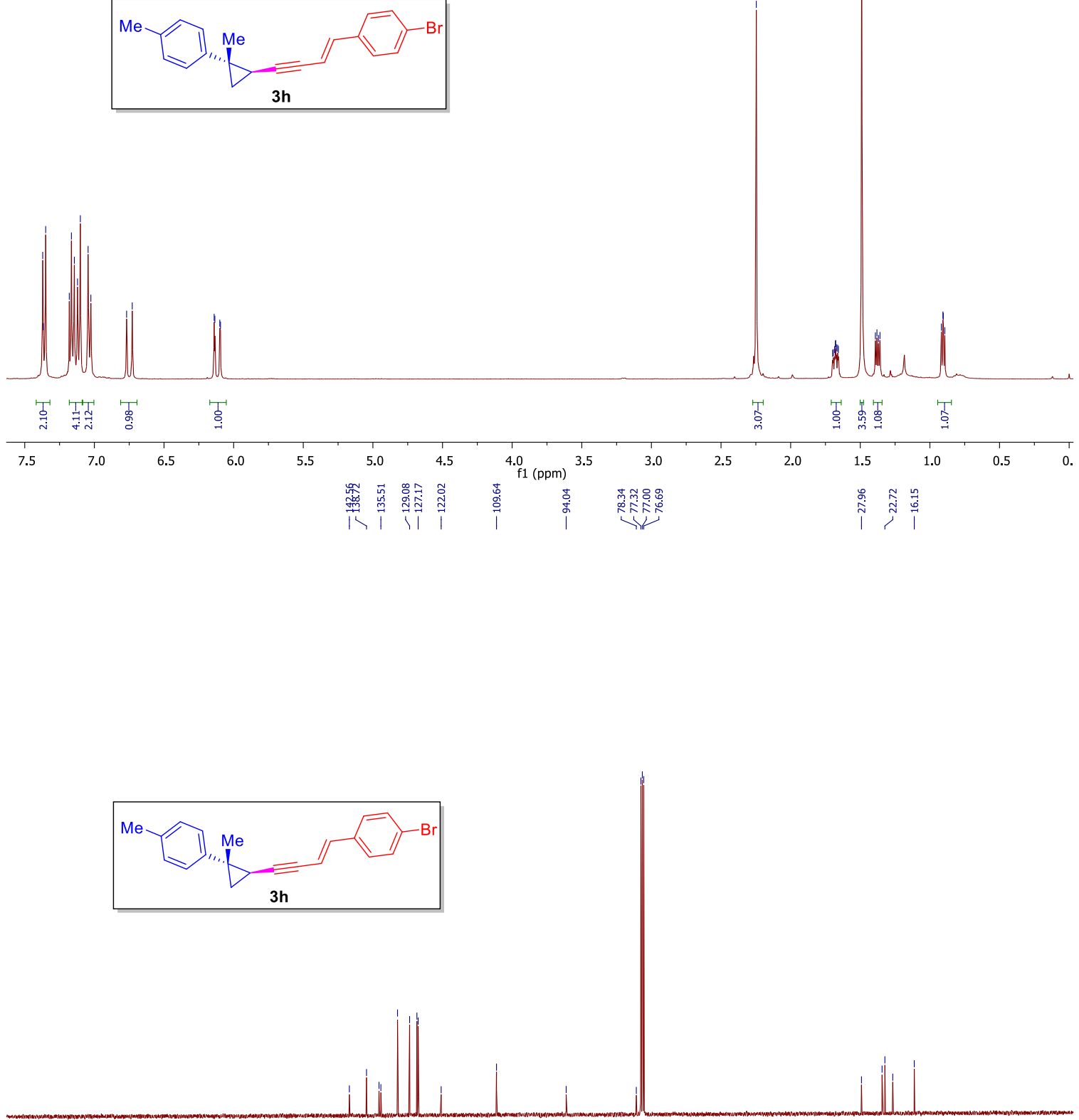

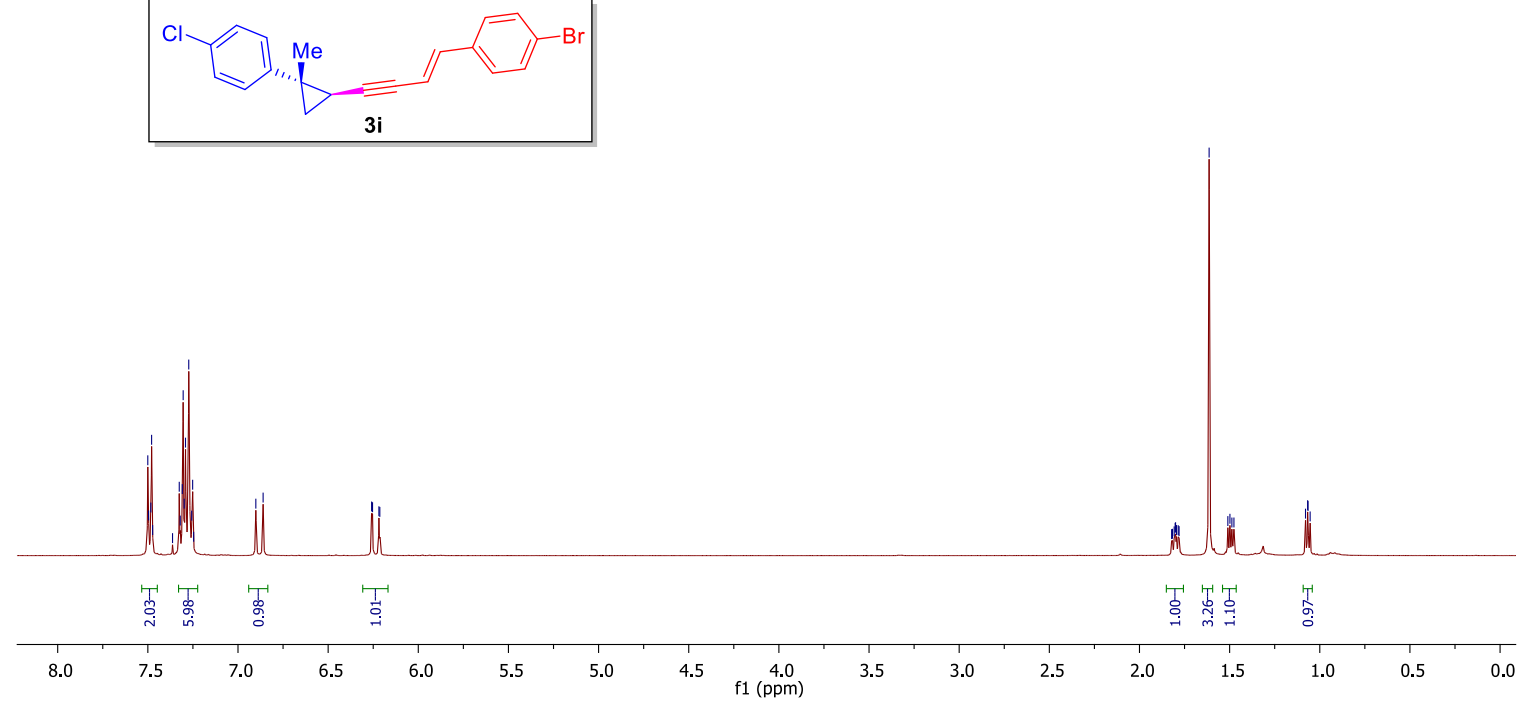

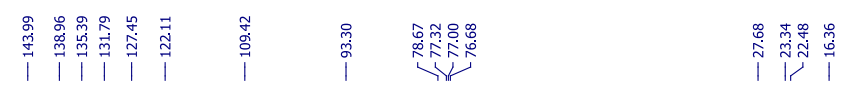
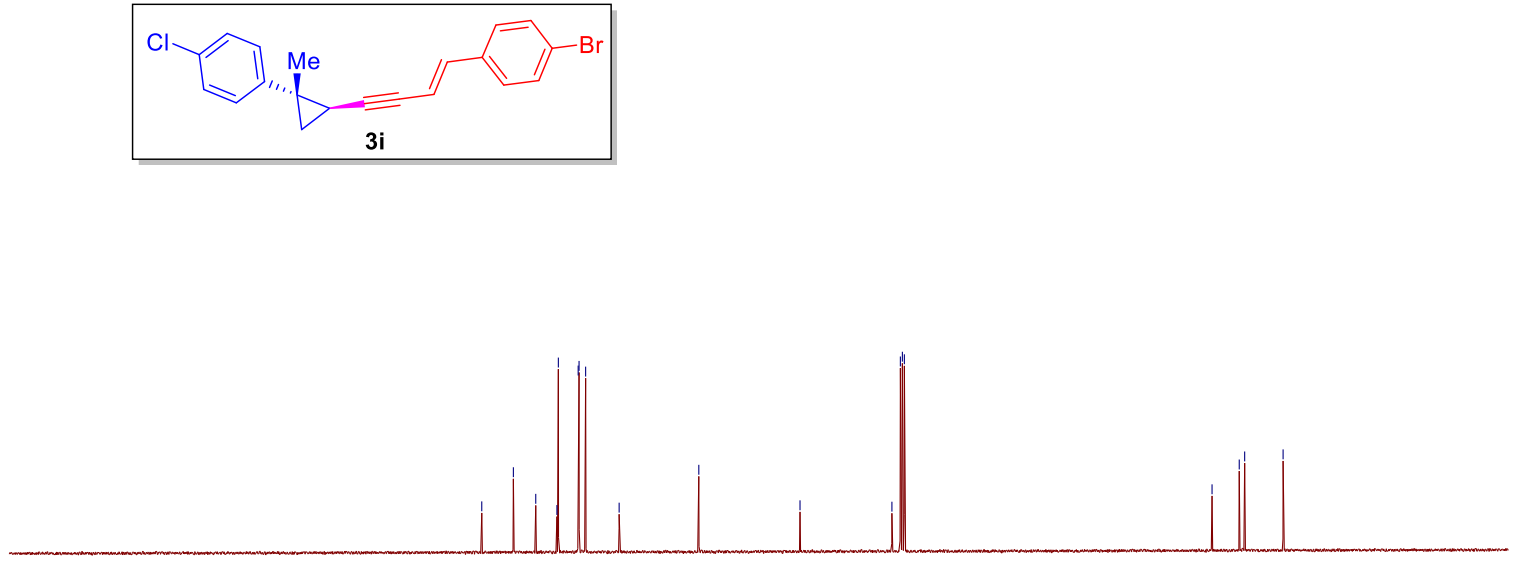
Supporting Information
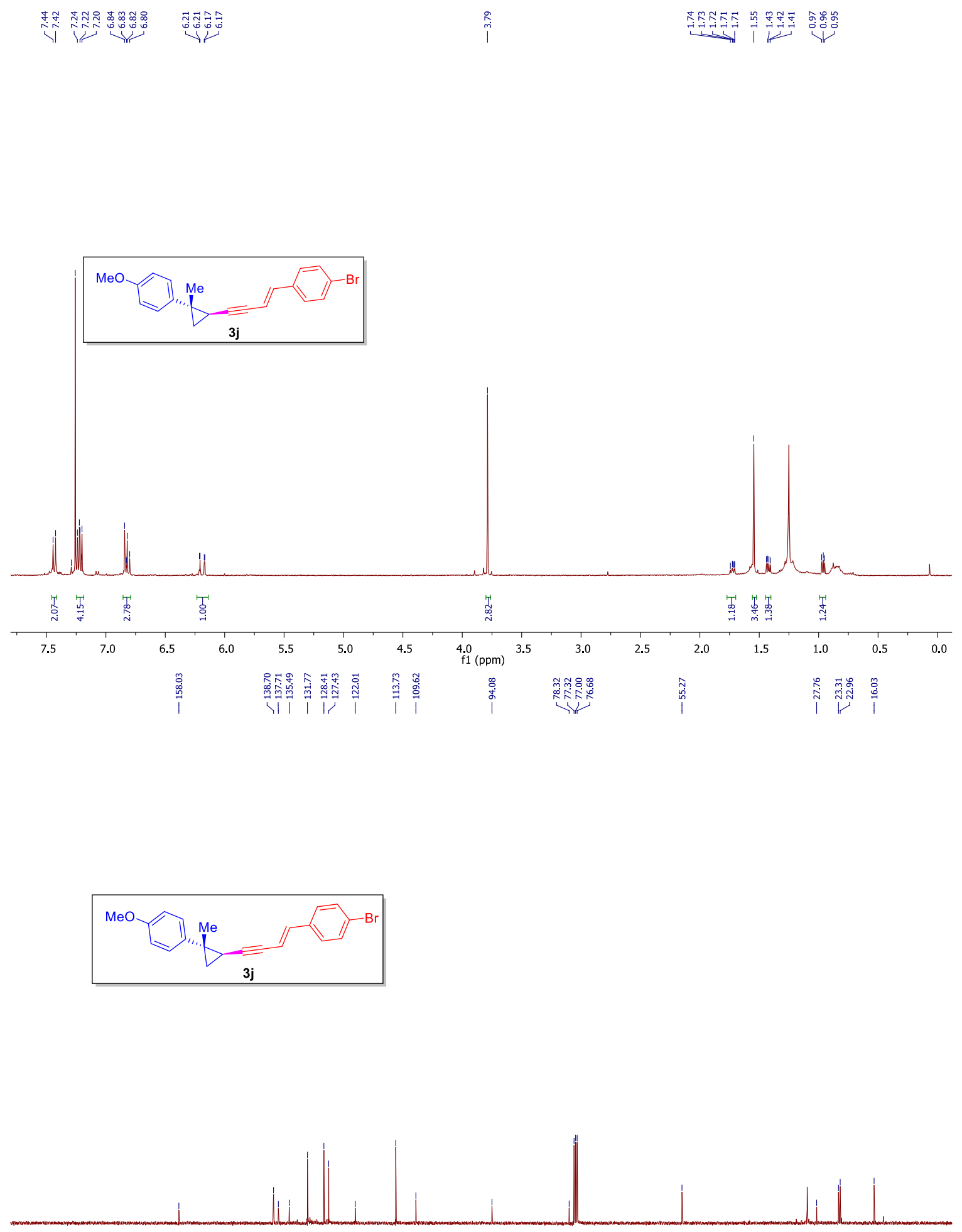
Supporting Information

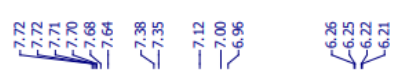

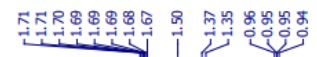
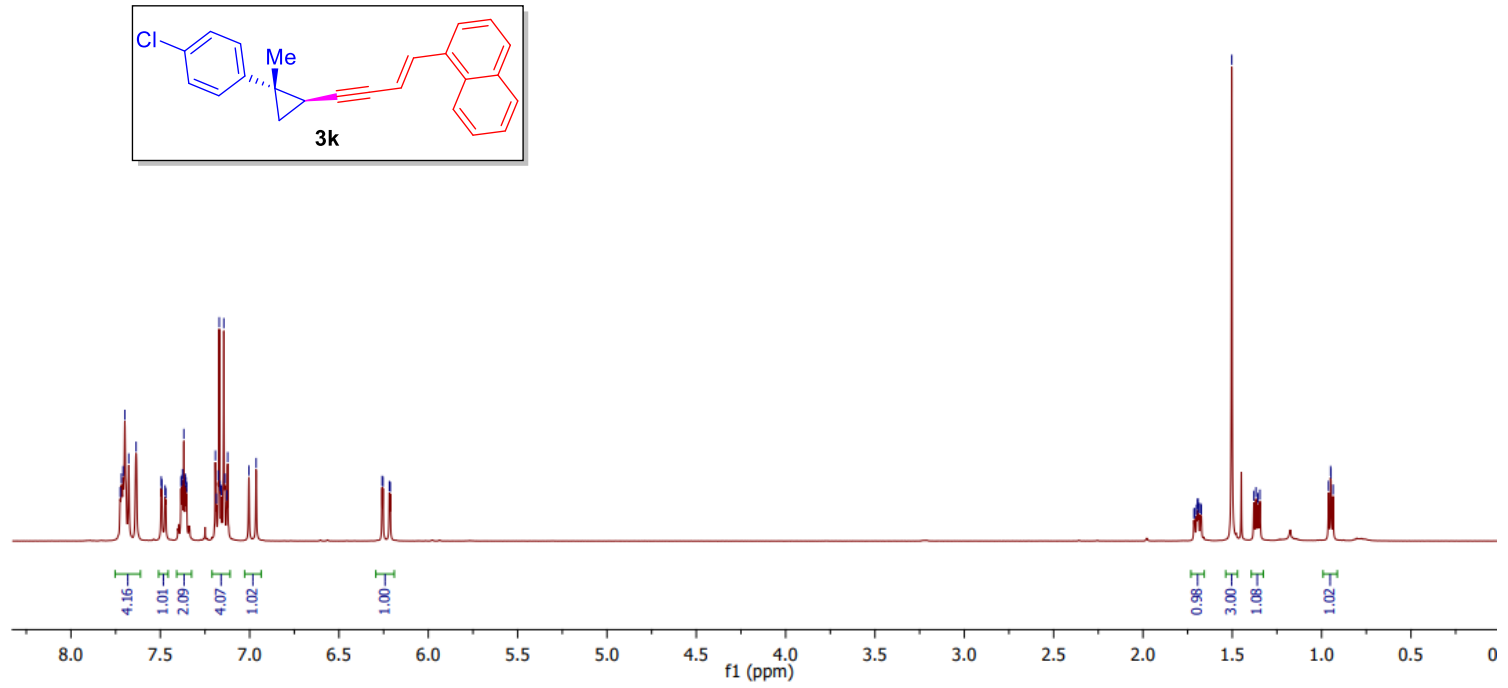

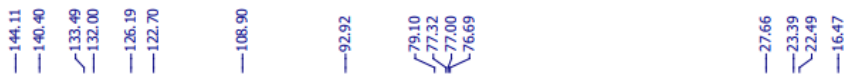
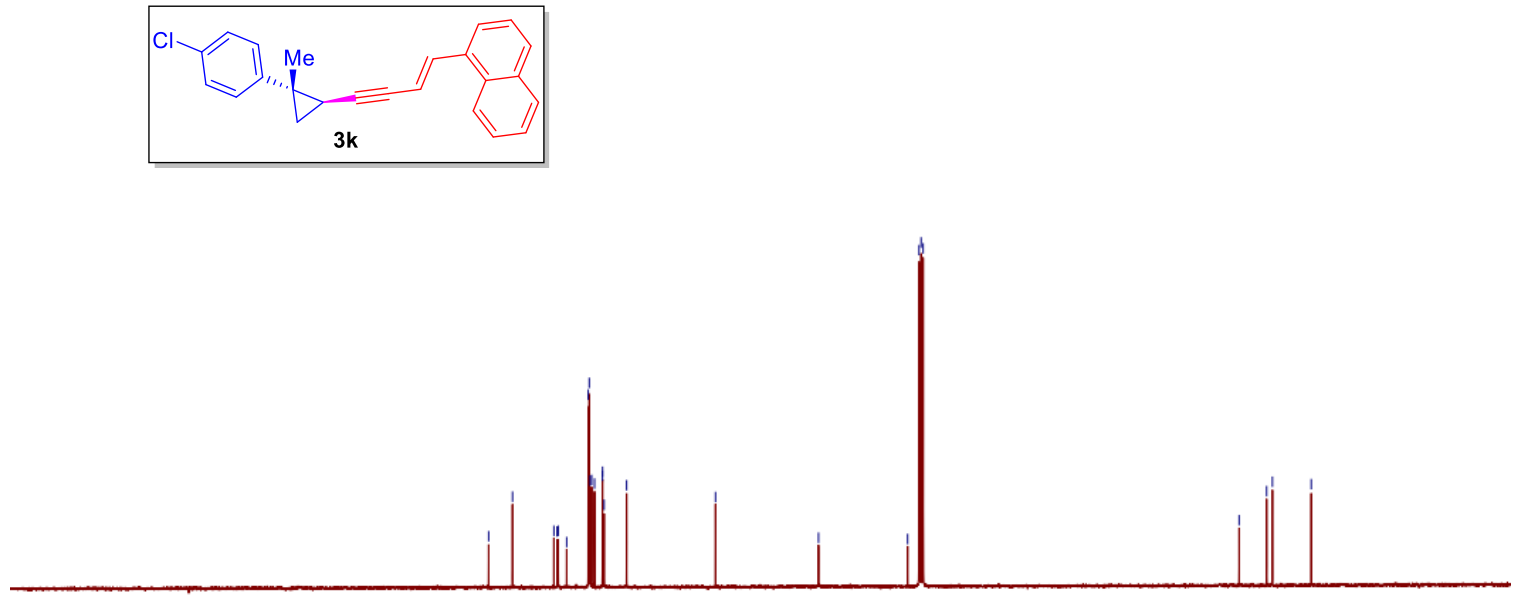

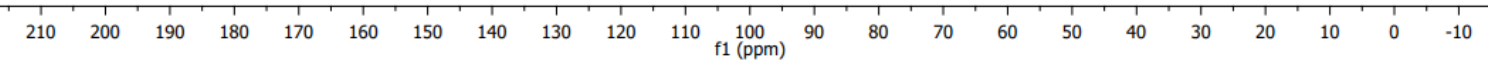




\section{5. $X$ ray Information of $2 s$}

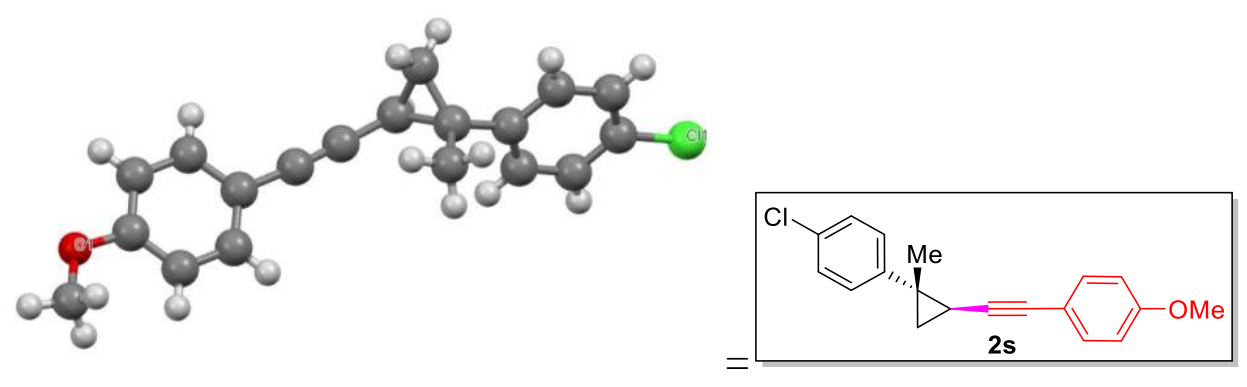

Table S5. Crystal data and structure refinement for Marek139b.

Identification code

Empirical formula

Formula weight

Temperature/K

Crystal system

Space group

$\mathrm{a} / \AA$

$\mathrm{b} / \AA$

$\mathrm{c} / \AA$

$\alpha /{ }^{\circ}$

$\beta /{ }^{\circ}$

$\gamma /{ }^{\circ}$

Volume/Å3

$\mathrm{Z}$

$\rho_{\text {calcg }} / \mathrm{cm}_{3}$

$\mu / \mathrm{mm}-1$

$\mathrm{F}(000)$

Crystal size/mm3

Radiation

$2 \Theta$ range for data collection/ ${ }^{\circ}$

Index ranges

Reflections collected

Independent reflections

Data/restraints/parameters

Goodness-of-fit on $\mathrm{F}_{2}$

Final R indexes $[\mathrm{I}>=2 \sigma(\mathrm{I})]$

Final $\mathrm{R}$ indexes [all data]

Largest diff. peak/hole / e Å-3

Flack parameter
Marek139b

$\mathrm{C}_{19} \mathrm{H}_{17} \mathrm{ClO}$

296.77

296.15

orthorhombic

$\mathrm{P} 212121$

$5.6242(13)$

$7.5222(18)$

37.072(9)

90

90

90

1568.4(6)

4

1.257

0.240

624.0

$0.3 \times 0.21 \times 0.18$

$\operatorname{MoK} \alpha(\lambda=0.71073)$

2.198 to 50.148

$-6 \leq \mathrm{h} \leq 6,-8 \leq \mathrm{k} \leq 8,-39 \leq 1 \leq 43$

9468

$2774\left[\mathrm{R}_{\text {int }}=0.0331, \mathrm{R}_{\text {sigma }}=0.0271\right]$

2774/0/192

1.052

$\mathrm{R}_{1}=0.0274, \mathrm{wR}_{2}=0.0639$

$\mathrm{R}_{1}=0.0292, \mathrm{wR}_{2}=0.0668$

$0.14 /-0.19$

$-0.02(2)$

Table S6. Fractional Atomic Coordinates $(\times 104)$ and Equivalent Isotropic 
Displacement Parameters $\left(\AA_{2} \times 103\right)$ for Marek139b. Ueq is defined as $1 / 3$ of of the trace of the orthogonalised UIJ tensor.

\begin{tabular}{|c|c|c|c|c|}
\hline Atom & $x$ & $y$ & $z$ & $\mathbf{U}(\mathbf{e q})$ \\
\hline $\mathrm{C} 1$ & $10893(5)$ & $5460(3)$ & $1691.2(6)$ & $49.6(7)$ \\
\hline $\mathrm{C} 2$ & $8357(4)$ & $6590(3)$ & $2157.3(5)$ & $27.0(5)$ \\
\hline $\mathrm{C} 3$ & $6267(4)$ & $7488(3)$ & $2234.5(5)$ & $30.7(5)$ \\
\hline $\mathrm{C} 4$ & $5387(4)$ & 7510 (3) & $2581.8(5)$ & $28.7(5)$ \\
\hline $\mathrm{C} 5$ & $6588(4)$ & $6622(3)$ & $2859.8(5)$ & $24.8(4)$ \\
\hline C6 & 8701 ( 4) & $5760(3)$ & $2776.2(5)$ & $27.9(5)$ \\
\hline $\mathrm{C} 7$ & $9585(4)$ & $5724(3)$ & $2428.5(5)$ & $27.7(5)$ \\
\hline $\mathrm{C} 8$ & $5588(4)$ & $6594(3)$ & $3217.2(6)$ & $30.4(5)$ \\
\hline C9 & $4611(4)$ & $6560(3)$ & $3504.4(6)$ & $32.9(5)$ \\
\hline C10 & $3372(4)$ & $6485(3)$ & $3844.8(5)$ & $33.0(5)$ \\
\hline C11 & $1094(4)$ & $5410(3)$ & $3874.8(6)$ & $40.7(6)$ \\
\hline C12 & $3276(4)$ & $4784(3)$ & $4065.8(5)$ & $27.7(5)$ \\
\hline C13 & $4527(5)$ & $3154(3)$ & $3920.6(6)$ & $41.1(6)$ \\
\hline C14 & $3386(3)$ & $4957(3)$ & $4469.6(5)$ & $25.5(4)$ \\
\hline C15 & $1652(4)$ & $4200(3)$ & $4686.2(5)$ & $29.7(5)$ \\
\hline C16 & $1804(4)$ & $4257(3)$ & $5059.7(6)$ & $32.2(5)$ \\
\hline $\mathrm{C} 17$ & $3725(4)$ & $5092(3)$ & $5215.5(5)$ & $29.3(5)$ \\
\hline C18 & $5480(4)$ & $5867(3)$ & $5009.2(6)$ & $32.5(5)$ \\
\hline C19 & $5296(4)$ & $5783(3)$ & $4637.1(6)$ & $30.6(5)$ \\
\hline $\mathrm{Cl1}$ & $3942.3(12)$ & $\begin{array}{l}5197.0( \\
8)\end{array}$ & $5685.1(2)$ & $\begin{array}{l}45.30(1 \\
8)\end{array}$ \\
\hline $\mathrm{O} 1$ & $9078(3)$ & $6673(2)$ & $1803.8(4)$ & $40.1(4)$ \\
\hline
\end{tabular}

Table S7. Anisotropic Displacement Parameters $\left(\AA_{2 \times 103}\right)$ for Marek139b. The Anisotropic displacement factor exponent takes the form: $2 \pi_{2}\left[h_{2} a *_{2} U_{11}+2 h k a * b * U_{12}+\ldots\right]$.

$\begin{array}{lllllll}\text { Atom U11 } & \mathbf{U 2 2} & \mathbf{U 3 3} & \mathbf{U} 23 & \mathbf{U 1 3} & \mathbf{U 1 2} \\ \text { C1 } & 60.2(16) & 60.1(16) & 28.6(12) & -3.1(11) & 13.8(12) & 12.5(15) \\ \text { C2 } & 36.1(12) & 23.7(10) & 21.1(10) & -0.1(9) & 2.2(9) & -3.5(10) \\ \text { C3 } & 36.7(12) & 29.5(11) & 25.9(11) & 3.6(9) & -6.5(10) & 3.3(11) \\ \text { C4 } & 27.5(12) & 27.8(11) & 30.8(12) & -0.8(9) & 1.4(10) & 2.3(10) \\ \text { C5 } & 29.9(11) & 23(1) & 21.4(10) & -0.6(8) & 1.9(9) & -4.0(9) \\ \text { C6 } & 32.1(11) & 27.8(11) & 24(1) & 3.8(8) & -2.8(9) & 1(1) \\ \text { C7 } & 27.1(11) & 27.8(10) & 28.3(11) & 0.4(9) & -0.1(9) & 2.2(9) \\ \text { C8 } & 34.2(12) & 27.8(11) & 29.2(12) & 0.5(9) & 2.3(10) & -1.1(10) \\ \text { C9 } & 39.0(13) & 30.3(11) & 29.3(12) & 1.5(10) & 2.6(10) & 2.7(11) \\ \text { C10 } & 40.3(13) & 32.3(12) & 26.3(12) & 1.1(9) & 8.2(10) & 4.0(11) \\ \text { C11 } & 33.9(11) & 60.8(15) & 27.4(11) & 1.9(10) & 2.9(10) & 2.2(13)\end{array}$




$\begin{array}{lllllll}\text { C12 } & 31.2(11) & 29.4(11) & 22.7(10) & -0.7(9) & 2.9(8) & -2.4(10) \\ \text { C13 } & 58.1(16) & 35.4(13) & 29.8(12) & -3.9(10) & 1.9(12) & 5.4(12) \\ \text { C14 } & 26.6(10) & 24.1(10) & 25.7(10) & 0.5(8) & 2.0(8) & 2.7(10) \\ \text { C15 } & 30.0(11) & 30.5(11) & 28.5(11) & -0.3(9) & -1.5(9) & -4.7(10) \\ \text { C16 } & 33.5(12) & 32.6(12) & 30.6(12) & 5.2(9) & 6.7(10) & -4.1(10) \\ \text { C17 } & 38.3(11) & 27.3(11) & 22.3(10) & -0.1(8) & -1.6(9) & 5.2(11) \\ \text { C18 } & 31.2(12) & 33.0(12) & 33.3(12) & -3.3(9) & -4.7(10) & -4.9(10) \\ \text { C19 } & 27.2(11) & 33.2(12) & 31.5(12) & 0.9(9) & 5.8(9) & -4.9(10) \\ \text { C11 } & 67.7(4) & 46.3(3) & 21.9(3) & 1.4(2) & -4.8(3) & -3.2(3) \\ \text { O1 } & 57.1(10) & 41.3(9) & 21.9(8) & 5.5(6) & 7.6(8) & 10.6(9)\end{array}$

Table S8. Bond Lengths for Marek139b.

\begin{tabular}{|c|c|c|c|c|c|}
\hline \multicolumn{3}{|c|}{ Atom Atom Length/̊̊ } & \multicolumn{3}{|c|}{ Atom Atom Length/Å } \\
\hline $\mathrm{C} 1$ & $\mathrm{O} 1$ & $1.431(3)$ & $\mathrm{C} 10$ & $\mathrm{C} 12$ & $1.521(3)$ \\
\hline $\mathrm{C} 2$ & $\mathrm{C} 3$ & $1.386(3)$ & $\mathrm{C} 11$ & $\mathrm{C} 12$ & $1.493(3)$ \\
\hline $\mathrm{C} 2$ & $\mathrm{C} 7$ & $1.383(3)$ & $\mathrm{C} 12$ & $\mathrm{C} 13$ & $1.513(3)$ \\
\hline $\mathrm{C} 2$ & $\mathrm{O} 1$ & $1.374(2)$ & $\mathrm{C} 12$ & C14 & $1.504(3)$ \\
\hline $\mathrm{C} 3$ & $\mathrm{C} 4$ & $1.379(3)$ & $\mathrm{C} 14$ & $\mathrm{C} 15$ & $1.386(3)$ \\
\hline $\mathrm{C} 4$ & C5 & $1.401(3)$ & $\mathrm{C} 14$ & C19 & $1.388(3)$ \\
\hline $\mathrm{C} 5$ & C6 & $1.389(3)$ & $\mathrm{C} 15$ & $\mathrm{C} 16$ & $1.388(3)$ \\
\hline C5 & C8 & $1.440(3)$ & C16 & $\mathrm{C} 17$ & $1.377(3)$ \\
\hline C6 & $\mathrm{C} 7$ & $1.382(3)$ & $\mathrm{C} 17$ & $\mathrm{C} 18$ & $1.378(3)$ \\
\hline $\mathrm{C} 8$ & C9 & $1.198(3)$ & $\mathrm{C} 17$ & $\mathrm{Cl} 1$ & $1.7471(19)$ \\
\hline C9 & $\mathrm{C} 10$ & $1.443(3)$ & $\mathrm{C} 18$ & C19 & $1.384(3)$ \\
\hline $\mathrm{C} 10$ & $\mathrm{C} 11$ & $1.519(3)$ & & & \\
\hline
\end{tabular}

Table S9. Bond Angles for Marek139b.

\begin{tabular}{lllllllll}
\multicolumn{3}{ll}{ Atom Atom Atom Angle $/^{\circ}$} & & \multicolumn{3}{l}{ Atom Atom Atom Angle $/^{\circ}$} \\
$\mathrm{C} 7$ & $\mathrm{C} 2$ & $\mathrm{C} 3$ & $120.22(18)$ & & $\mathrm{C} 11$ & $\mathrm{C} 12$ & $\mathrm{C} 13$ & $118.01(19)$ \\
$\mathrm{O} 1$ & $\mathrm{C} 2$ & $\mathrm{C} 3$ & $115.18(18)$ & & $\mathrm{C} 11$ & $\mathrm{C} 12$ & $\mathrm{C} 14$ & $118.60(18)$ \\
$\mathrm{O} 1$ & $\mathrm{C} 2$ & $\mathrm{C} 7$ & $124.59(19)$ & & $\mathrm{C} 13$ & $\mathrm{C} 12$ & $\mathrm{C} 10$ & $118.27(17)$ \\
$\mathrm{C} 4$ & $\mathrm{C} 3$ & $\mathrm{C} 2$ & $120.18(19)$ & & $\mathrm{C} 14$ & $\mathrm{C} 12$ & $\mathrm{C} 10$ & $117.51(18)$ \\
$\mathrm{C} 3$ & $\mathrm{C} 4$ & $\mathrm{C} 5$ & $120.53(19)$ & & $\mathrm{C} 14$ & $\mathrm{C} 12$ & $\mathrm{C} 13$ & $113.90(18)$ \\
$\mathrm{C} 4$ & $\mathrm{C} 5$ & $\mathrm{C} 8$ & $119.71(18)$ & & $\mathrm{C} 15$ & $\mathrm{C} 14$ & $\mathrm{C} 12$ & $120.81(18)$ \\
$\mathrm{C} 6$ & $\mathrm{C} 5$ & $\mathrm{C} 4$ & $118.09(18)$ & & $\mathrm{C} 15$ & $\mathrm{C} 14$ & $\mathrm{C} 19$ & $117.99(18)$ \\
$\mathrm{C} 6$ & $\mathrm{C} 5$ & $\mathrm{C} 8$ & $122.19(18)$ & & $\mathrm{C} 19$ & $\mathrm{C} 14$ & $\mathrm{C} 12$ & $121.09(18)$ \\
$\mathrm{C} 7$ & $\mathrm{C} 6$ & $\mathrm{C} 5$ & $121.66(19)$ & & $\mathrm{C} 14$ & $\mathrm{C} 15$ & $\mathrm{C} 16$ & $121.5(2)$ \\
$\mathrm{C} 6$ & $\mathrm{C} 7$ & $\mathrm{C} 2$ & $119.30(19)$ & & $\mathrm{C} 17$ & $\mathrm{C} 16$ & $\mathrm{C} 15$ & $118.75(19)$
\end{tabular}




\begin{tabular}{|c|c|c|c|c|c|c|c|}
\hline C9 & $\mathrm{C} 8$ & $\mathrm{C} 5$ & $175.7(2)$ & $\mathrm{C} 16$ & C17 & C18 & $121.48(19)$ \\
\hline $\mathrm{C} 8$ & C9 & $\mathrm{C} 10$ & $178.1(2)$ & $\mathrm{C} 16$ & $\mathrm{C} 17$ & $\mathrm{Cl1}$ & $119.57(16)$ \\
\hline 59 & $\mathrm{C} 10$ & $\mathrm{C} 11$ & $119.49(19)$ & C18 & C17 & $\mathrm{Cl1}$ & $(16)$ \\
\hline C9 & $\mathrm{C} 10$ & $\mathrm{C} 12$ & $121.43(19)$ & $\mathrm{C} 17$ & C18 & C19 & $18.7(2)$ \\
\hline C11 & $\mathrm{C} 10$ & $\mathrm{C} 12$ & $58.82(14)$ & C18 & C19 & C14 & $6(2)$ \\
\hline $\mathrm{C} 12$ & C11 & $\mathrm{C} 10$ & $60.65(15)$ & $\mathrm{C} 2$ & $\mathrm{O} 1$ & $\mathrm{C} 1$ & $117.38(1$ \\
\hline 11 & $\mathrm{C} 12$ & $\mathrm{C} 10$ & $60.53(15)$ & & & & \\
\hline
\end{tabular}

Table S10. Hydrogen Atom Coordinates $(\AA \times 104)$ and Isotropic Displacement Parameters $\left(\AA_{2 \times 103}\right)$ for Marek139b.

$\begin{array}{lllll}\text { Atom } \boldsymbol{x} & \boldsymbol{y} & \boldsymbol{z} & \mathbf{U}(\mathbf{e q}) \\ \text { H1A } 11181 & 5604 & 1432 & 74 \\ \text { H1B } 10378 & 4239 & 1740 & 74 \\ \text { H1C } 12361 & 5707 & 1824 & 74 \\ \text { H3 } 5438 & 8091 & 2048 & 37 \\ \text { H4 } 3955 & 8131 & 2633 & 34 \\ \text { H6 } 9561 & 5181 & 2963 & 34 \\ \text { H7 } 11021 & 5111 & 2376 & 33 \\ \text { H10 3402 } & 7610 & 3989 & 40 \\ \text { H11A }-210 & 5907 & 4023 & 49 \\ \text { H11B } 570 & 4736 & 3660 & 49 \\ \text { H13A } 4340 & 3106 & 3658 & 62 \\ \text { H13B 3831 } & 2085 & 4029 & 62 \\ \text { H13C 6222 } & 3215 & 3981 & 62 \\ \text { H15 329 } & 3629 & 4577 & 36 \\ \text { H16 } 606 & 3729 & 5205 & 39 \\ \text { H18 6790 } & 6446 & 5120 & 39 \\ \text { H19 } 6507 & 6305 & 4493 & 37\end{array}$

\section{Experimental}

The crystal was kept at $296.15 \mathrm{~K}$ during data collection. Using Olex2 [1], the structure was solved with the SIR2004 [2] structure solution program using Direct Methods and refined with the ShelXL [3] refinement package using Least Squares minimisation.

1. Dolomanov, O.V., Bourhis, L.J., Gildea, R.J, Howard, J.A.K. \& Puschmann, H. (2009), J. Appl. Cryst. 42, 339-341.

2. Burla, M.C., Caliandro, R., Camalli, M., Carrozzini, B., Cascarano, G.L., De Caro, L., Giacovazzo, C., Polidori, G., Siliqi, D., Spagna, R. (2007). J. Appl. Cryst. 40, 609-613.

3. Sheldrick, G.M. (2015). Acta Cryst. C71, 3-8.

\section{Crystal structure determination of [Marek139b]}

Crystal Data for $\mathrm{C}_{19} \mathrm{H}_{17} \mathrm{ClO}(M=296.77 \mathrm{~g} / \mathrm{mol})$ : orthorhombic, space group $\mathrm{P} 2{ }_{12}{ }_{12}{ }_{1}$ (no. 19), $a=$ 5.6242(13) $\AA, b=7.5222(18) \AA, c=37.072(9) \AA, V=1568.4(6) \AA 3 ., Z=4, T=296.15 \mathrm{~K}, \mu(\mathrm{MoK} \alpha)=$ $0.240 \mathrm{~mm}-1$, Dcalc $=1.257 \mathrm{~g} / \mathrm{cm}_{3}, 9468$ reflections measured $\left(2.198^{\circ} \leq 2 \Theta \leq 50.148^{\circ}\right), 2774$ unique $(R$ int $=$ 
0.0331, Rsigma $=0.0271)$ which were used in all calculations. The final $R 1$ was 0.0274 (I $>2 \sigma(\mathrm{I})$ ) and $w R_{2}$ was 0.0668 (all data).

\section{Refinement model description}

Number of restraints - 0 , number of constraints - unknown.

Details:

1 .

At

All $\quad \mathrm{C}(\mathrm{H})$

At

All

$\mathrm{C}(\mathrm{H})$

2.a Ternary

C1O (H10)

2.b Secondary

C11 (H11A, H11B)

2. C Aromatic/amid

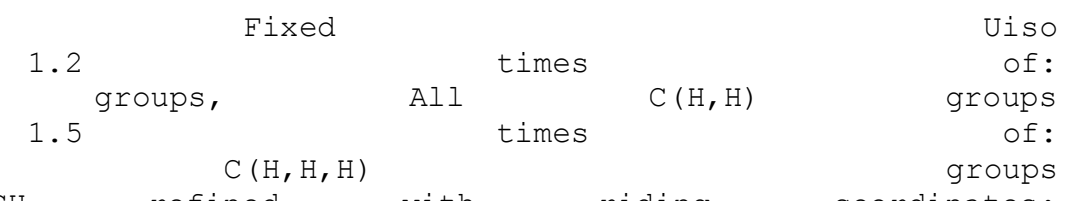

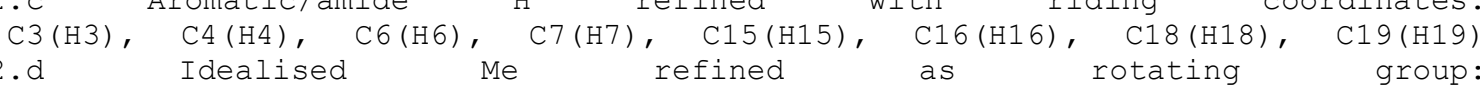

C1 (H1A, H1B, H1C), C13 (H13A, H13B, H13C) 